\title{
Stratified and Stirred: \\ Monsoon Freshwater in the Bay of Bengal
}

\author{
by \\ Gualtiero Victor Rudi Spiro Jaeger
}

B.S. Physics, CCS, University of California Santa Barbara (2013)

Submitted to the

Joint Program in Oceanography / Applied Ocean Science \& Engineering

in partial fulfillment of the requirements for the degree of

Doctor of Philosophy

at the

MASSACHUSETTS INSTITUTE OF TECHNOLOGY

and the

WOODS HOLE OCEANOGRAPHIC INSTITUTION

June 2019

(C)2019 Gualtiero V. R. Spiro Jaeger.

All rights reserved.

The author hereby grants to MIT and WHOI permission to reproduce and to distribute publicly paper and electronic copies of this thesis document in whole or in part in any medium now known or hereafter created.

Author

Joint Program in Oceanography / Applied Ocean Science \& Engineering

Massachusetts Institute of Technology \& Woods Hole Oceanographic Institution

March 21, 2019

Certified by

Dr. Amala Mahadevan

Senior Scientist, Woods Hole Oceanographic Institution

Thesis Supervisor

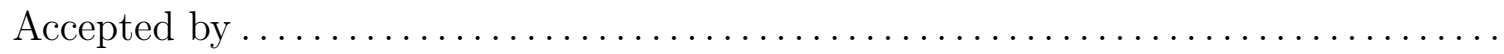

Prof. Glenn R. Flierl

Chairman, Joint Committee for Physical Oceanography

Massachusetts Institute of Technology \& Woods Hole Oceanographic Institution 


\title{
Stratified and Stirred:
}

\section{Monsoon Freshwater in the Bay of Bengal}

\author{
by \\ Gualtiero Victor Rudi Spiro Jaeger
}

Submitted to the Joint Program in Oceanography / Applied Ocean Science \& Engineering Massachusetts Institute of Technology \& Woods Hole Oceanographic Institution on March 21, 2019, in partial fulfillment of the requirements for the degree of

Doctor of Philosophy

\begin{abstract}
Submesoscale ocean dynamics and instabilities, with characteristic scales $0.1-10 \mathrm{~km}$, can play a critical role in setting the ocean's surface boundary layer thickness and associated density stratification. Submesoscale instabilities contribute to lateral stirring and tracer dispersal. These dynamics are investigated in the Bay of Bengal, motivated by the upper ocean's potentially coupled interactions with Monsoon winds and convection. The region's excess precipitation and runoff generates strong salinity gradients that typically set density fronts and stratification in the upper $50 \mathrm{~m}$. Since we cannot synoptically measure currents containing fast-evolving and oscillating components across the submesoscale range, we instead analyze passive tracer distributions (spice $\equiv$ density-compensated temperature $(T)$ and salinity $(S)$ anomalies), identifying signatures of flows and testing dynamical theories. The analysis is based on over 9000 vertical profiles of $T$ and $S$ measured along $\sim 4800 \mathrm{~km}$ of ship tracks in the Bay of Bengal during ASIRI and MISO-BOB expeditions in 2013, 2015, and 2018. Observations in the surface mixed layer reveal $\sim 1 \mathrm{~km}$ scale-selective correlation of surface $T$ and $S$, with compensation reducing cross-front density gradients by $\sim 50 \%$. Using a process study ocean model, we show this is caused by submesoscale instabilities slumping fronts, plus surface cooling over the resultant enhanced salinity stratification, potentially thwarting the forward cascade of energy. In the stratified interior, we present a spectral analysis of horizontal spice variance statistics from wavenumber $k \sim 0.01 \mathrm{cpkm}$ to $\sim 1 \mathrm{cpkm}$. At scales $<10 \mathrm{~km}$, stratified layers that are closer to the surface exhibit redder passive tracer spectra (power spectra $k^{-3}$, gradient spectra $k^{-1}$ ) than predicted by quasi-geostrophic or frontogenetic theories. Complimentary observations reveal spice patterns with multiple, parallel, $\sim 10 \mathrm{~m}$ thin layers, crossing isopycnals with $O\left(10^{-4}\right)$ slopes, coherent over at least 30-80 km, with coincident layers of stratification anomalies. Comparison with shear measurements, and a numerical process study, suggest that both submesoscale sheared eddies, and thin near-inertial waves, form such layers. Fast formation timescales and large aspect ratios suggest they enhance horizontal mixing by shear dispersion, reducing variance at $\sim 1-10 \mathrm{~km}$ scales.
\end{abstract}

Thesis Supervisor: Dr. Amala Mahadevan

Title: Senior Scientist, Woods Hole Oceanographic Institution 


\section{Acknowledgments}

First and foremost, this thesis would not exist without the support, advice, and encouragement I received from my adviser, A.Mahadevan. Thank you for your infectious energy, your curiosity, your natural mentorship, and your tolerance of my many extra-curricular activities and pursuits. It has been a pleasure to investigate the Bay of Bengal's mysteries.

Thank you to the members on my thesis committee, as well as the great chair of my defense G.Flierl, for guiding my thesis journey with much insight, support, and especially patience. In addition to sharing your expertise in oceanography, I have benefited and hopefully picked up a thing or two from your expert styles. I strive to approach E.Shroyer's calm and focused leadership of high-stakes expeditions with vivacity, J.T.Farrar's thoroughness and persistence in pursuing goals, and R.Ferrari's seemingly intuitive ability to spot the critical issue relevant to every big picture.

I'm grateful to have sailed with an outstanding crew on research expeditions, including captain, ship crew, and fellow scientists on the $R / V$ Revelle and the $R / V$ Thompson. A fantastically fun team to be a part of, through tropical downpours and muggy nights. My gratitude extends to all ASIRI-OMM and MISO-BOB scientists, including A.Lucas, J.MacKinnon, A.Tandon, D.Sengupta, E.D'Asaro, J.Nash, H.Seo, M.Omand, A.Subramanian, S.Nguyen, J.Ladner, J.Buckley, and many more, for their advice, collaboration, and collegiality over the many years of the project, as well as their encouragement of my work, especially from T. Paluszkiewicz at ONR.

This work was carried out with the support of the Office of Naval Research's "AirSea Interaction Regional Initiative - ASIRI" (Grants N000141612470 and N000141310451) as well as the "Monsoon Intra-Seasonal Oscillations in the Bay of Bengal - MISO-BOB" research initiative (Grant N0000141712390), in collaboration with the Indian Ministry of Earth Science's "Ocean Mixing and Monsoons - OMM" initiative supported by the "Monsoon Mission".

This work was also invaluably supported by my family and friends, whose steadfast trust, encouragement, and company along the $\mathrm{PhD}$ path made it all possible. Whether sitting on a dock on the Charles on a Boston night, or sitting on a dock in Woods Hole at the end of a sunny day, or on a rooftop deck, sail boat, research ship, campus bar, hotel bar, or wherever else a cool refreshment was at hand, the friends I met, and made for life, made the journey worthwhile. Thank you for friendships that will keep reuniting us long from now, when memories of the $\mathrm{PhD}$ roller coaster will bring us back to those docks.

Lastly, I wouldn't be writing this had my parents not steadfastly nourished my curiosity and education throughout the years, and shared the joy and fascination of exploring the sea and its thresholds, from Saint-Florent, via starry nights in sleeping bags on ferry decks, out west to the Pacific, and back east to the Atlantic.

This thesis is dedicated to Dr. Anneliese Spiro Kern, the best grandmother on this planet, and best travel buddy a young boy can have to embark on joint expeditions, from digging up fossils and panning gold in the countryside, to eating dates and taking baths in ancient Phoenician cities, to visiting the land of trolls. With love, G.V.R.S.J. 
THIS PAGE INTENTIONALLY LEFT BLANK 


\section{Contents}

$\begin{array}{ll}\text { Introduction } & 11\end{array}$

1 Submesoscale-Selective Compensation of Fronts in a Salinity-Stratified $\begin{array}{ll}\text { Ocean } & 17\end{array}$

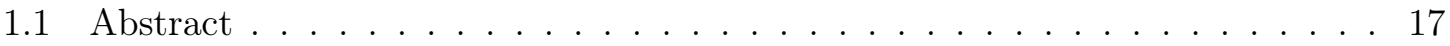

1.2 Introduction . . . . . . . . . . . . . . . . . . . . 18

1.3 Materials and Methods . . . . . . . . . . . . . . . . . . . 20

1.3.1 Remote Observations . . . . . . . . . . . . . . . . 20

1.3.2 Ship-based Observations . . . . . . . . . . . . . . . . . 22

1.4 Analysis . . . . . . . . . . . . . . . . . . . . 25

1.4.1 Three-dimensional Modeling . . . . . . . . . . . . . . . . 28

1.5 Model Results . . . . . . . . . . . . . . . . . . . . . . . . . . . . . . 29

1.6 Discussion . . . . . . . . . . . . . . . . . . . . 31

1.6.1 Geographic Occurrence . . . . . . . . . . . . . . . . 31

1.6.2 Compensation Time Scale . . . . . . . . . . . . . . . . . 32

1.7 Concluding Remarks . . . . . . . . . . . . . . . . . . . . 34

1.8 Chapter Acknowledgments . . . . . . . . . . . . . . . . . . . . 35

1.9 Supplementary Material . . . . . . . . . . . . . . . . . . 36

2 How Spice is Stirred in the Bay of Bengal $\quad 47$

2.1 Abstract . . . . . . . . . . . . . . . . . . 47

2.2 Introduction $\ldots \ldots \ldots \ldots \ldots \ldots \ldots \ldots \ldots$

2.3 Oceanic Variance Spectra . . . . . . . . . . . . . . . . . . . . . . 49

2.4 Observations in the Bay of Bengal . . . . . . . . . . . . . . . 51

2.4 .1 Environment . . . . . . . . . . . . . . . . . 52 
2.4 .2 Measurement of $T$ and $S \ldots \ldots \ldots \ldots \ldots$

2.4 .3 Hydrography of the Upper $200 \mathrm{~m} \ldots \ldots$. . . . . . . . . . . 56

2.5 Spectral Analysis Methods . . . . . . . . . . . . . . . . . . 58

2.6 Results: Tracer Spectra . . . . . . . . . . . . . . . . . . . 64

2.7 Discussion . . . . . . . . . . . . . . . . . . 66

2.7.1 Contextualizing the Bay of Bengal Study _ . . . . . . . . . . . 66

2.7.2 Interpreting Bay of Bengal Spectra . . . . . . . . . . . . . . . . . . 69

2.7.3 Potential Explanations . . . . . . . . . . . . . . 71

2.8 Concluding Remarks . . . . . . . . . . . . . . . . . . . . 75

2.9 Supplementary Figures . . . . . . . . . . . . . . . . . . . . . . . . 77

3 Formation of Interleaving Layers in the Bay of Bengal $\quad 81$

3.1 Abstract . . . . . . . . . . . . . . . . . . . . 81

3.2 Introduction . . . . . . . . . . . . . . . . . . . . 82

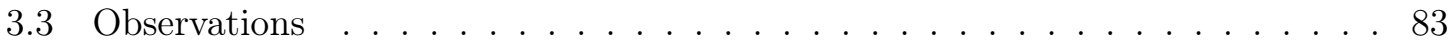

3.3 .1 Data Collection . . . . . . . . . . . . . . . 83

3.3.2 Data Processing . . . . . . . . . . . . . . . . . . . 84

3.3.3 Hydrography and Mesoscale Setting . . . . . . . . . . . . 86

3.4 Analysis of Layering Patterns . . . . . . . . . . . . . . . . . . . . 88

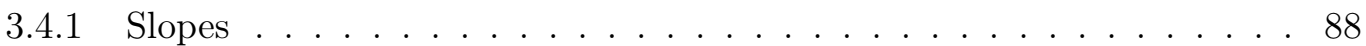

3.4 .2 Coincident Stratification Layering . . . . . . . . . . . . . . . . . 91

3.4 .3 Multiple Parallel Layers . . . . . . . . . . . . . . . . . . . . . . . 91

3.5 Mechanisms for Layer Formation _ . . . . . . . . . . . . . . . . . . 92

3.5.1 Existing Theory: Mesoscale Stirring of Tracer . . . . . . . . . . . . . . 92

3.5.2 New Hypothesized Layering Mechanisms in the Bay of Bengal . . . . . 94

3.5.3 Hypothesized Mechanism A: Submesoscale Sheared Eddy Twist . . . . 95

3.5.4 Hypothesized Mechanism B: Near-Inertial Wave Shear . . . . . . . . . 99

3.6 Discussion . . . . . . . . . . . . . . . . . . . . . . . . 101

3.6.1 Implications for Mixing in the Bay of Bengal . . . . . . . . . . . 103

3.7 Summary . . . . . . . . . . . . . . . . . . . . . 105

$\begin{array}{ll}\text { Conclusion } & 107\end{array}$ 


\section{List of Figures}

$0-1 \quad$ Salinity in the northern Indian Ocean $\ldots \ldots \ldots \ldots$

$0-2 \quad$ Spice and paint analogy $\ldots \ldots \ldots \ldots \ldots \ldots \ldots$

1-1 Schematic drawing of submesoscale density-compensation mechanism . . . . . 20

1-2 Winter-monsoon sea surface conditions in the northern Indian Ocean . . . . . 22

1-3 Surface-layer salinity and temperature structure in the Bay of Bengal . . . . . 24

1-4 Scale dependence of T-S compensation . . . . . . . . . . . . . 26

1-5 Numerical simulation of a submesoscale salinity front . . . . . . . . . . . . 30

1-6 Estimate of the global prevalence of cooling-induced T-S compensation . . . . 33

1-7 Idealized SSS submesoscale front, slumped and restratified . . . . . . . . . . 34

1-8 Supplementary - winter monsoon 2015 surface conditions . . . . . . . . . . . 37

1-9 Supplementary - scatterplots of density variability at wavelength of $0.6 \mathrm{~km}$. . 38

1-10 Supplementary - 1-D column models of active compensation mechanism . . . 40

1-11 Supplementary - paramter-configuration space of sensitivity tests . . . . . . 41

1-12 Supplementary - model's sensitivity to horizontal diffusivity . . . . . . . . . . 42

1-13 Supplementary - model's sensitivity to horizontal grid resolution . . . . . . . 43

1-14 Supplementary - model tests run with 2 different cooling scenarios . . . . . . 44

1-15 Supplementary - schematic of simple salinity fronts and stratification . . . . . 45

2-1 Schematic of passive tracer spectral distributions . . . . . . . . . . . . 52

2-2 The Bay of Bengal's surface salinity and subsurface oxygen saturation . . . . 53

2-3 Tracks of 3 cruises, currents, and climatological spice . . . . . . . . . . . 54

2-4 Mean vertical stratification profiles, temperature-salinity plot . . . . . . . . 57

2-5 Depth vs. distance sections of spice from cruises in the Bay of Bengal . . . . 59

2-6 Spectral analysis calculations along different isopycnals . . . . . . . . . . . 60 
2-7 Combining spectral estimates at equal densities along different sections . . . . 62

2-8 Salinity along isopycnals, and statistics of tracer gradient scales . . . . . . . 63

2-9 Comparison of tracer variance wavenumber spectra at different depths . . . . 65

2-10 Global map of eddy kinetic energy with locations of isopycnal spice studies . . 67

2-11 Schematic of the observed change in spectral slope of spice variance . . . . . . 72

2-12 Strong submesoscale density fronts in the surface mixed layer . . . . . . . . 73

2-13 Supplementary - surface chlorophyl-a, $100 \mathrm{~m} \mathrm{O}_{2}$ saturation, SSS . . . . . . . . 77

2-14 Supplementary - depth vs. distance sections of spice in 2013, 2015, 2018 . . . 78

2-15 Supplementary - wavelet decomposition . . . . . . . . . . . . . . . 79

3-1 Map of SLA, geostrophic currents, and cruise tracks from Sep. 2015 . . . . . 85

3-2 Lateral view of hydrographic sections . . . . . . . . . . . . . . . 87

3-3 Details of hydrographic sections . . . . . . . . . . . . . . . . . . . 89

3-4 Slopes of passive watermass tracer layers . . . . . . . . . . . . . . . . . 90

3-5 Vertical $T S$ and $N^{2}$ profiles . . . . . . . . . . . . . . . . . . . . 92

$3-6$ Properties of the prominent layering $\ldots \ldots \ldots$. . . . . . . . . . 93

3-7 Repeat transects across the fast jet . . . . . . . . . . . . . . . 96

3-8 Schematic and simulation of layering by submesoscale sheared eddy twist . . . 98

3-9 Schematic of layering by near-inertial wave shear . . . . . . . . . . . . . 102

3-10 Table summarizing layering mechanisms . . . . . . . . . . . . . . . 103

4-1 Schematic connecting freshwater and dynamics in the Bay of Bengal . . . . 108 


\section{Introduction}

It is easily recognized, on an abstract level, that a wide range of spatial scales are necessary for describing the ocean. Just how disparate the scales of important dynamical flow features and an ocean basin can be, is also easily lost in abstraction. The disparity was viscerally appreciated aboard a research vessel conducting fieldwork for this thesis, while located over 500 kilometers from the nearest coastline and river yet crossing a 10-meter-sharp horizontal gradient from saltier into fresher water of riverine origin. The discovery behind the sharp front, floating on top of 2000 meters of salty seawater, of a 5-meter-thin layer of distinctly fresher water emphasized the difficulty of including the dynamics that sharpen and stratify such small-scale features in global models. Yet the task is important, because the thickness, or in this case relative thinness, of the ocean's surface boundary layer determines the ocean's heat capacity on timescales relevant to weather and seasonal atmospheric phenomena, modulating the response of sea surface temperature (SST) to air-sea heat fluxes (Slingo et al., 2003). Especially over the tropical oceans, even relatively small $1^{\circ} \mathrm{C}$ changes in SST can profoundly affect atmospheric convection, rainfall, and wind patterns (Tompkins, 2001). Thus the vertical stratification of the upper ocean is of direct relevance to understanding and predicting atmospheric behaviour.

Boundary layer stratification can be a function of both external forcing parameters, and internal ocean dynamics (McPhaden, 2002). Of particular interest to surface stratification, and an active field of research in the zoo of geophysical fluid dynamics, is submesoscale turbulence (Callies et al., 2015). Named in relation to mesoscale motions, which at midlatitudes are dominated by eddies of order $100 \mathrm{~km}$ size, submesoscale flows have smaller characteristic horizontal scales, at mid-latitudes usually between $O(0.1)$ to $O(10) \mathrm{km}$. Alternatively and more accurately, submesoscale phenomena are defined dynamically as flows 
with order 1 Rossby and Richardson numbers, less constrained by Earth's rotation than mesoscale dynamics but more constrained than more 3-dimensional internal gravity waves. Submesoscale macro-turbulence became widely appreciated starting in the 2000's as playing an important role in shaping the properties of the upper ocean, yet is not fully understood (McWilliams, 2016).

The work in this thesis shows the relevance of submesoscales to the Bay of Bengal, the setting of the aforementioned hydrographic sampling (and musings on ocean scales). The Bay of Bengal is one of the two large basins, together with the Arabian Sea, forming the northern though tropical extent of the Indian Ocean. Even though surrounded by a population amounting to billions, it is a relatively under-explored region in terms of oceanographic research, especially relative to the northern Atlantic and Pacific basins. Characterised by an excess of river runoff and rainfall over evaporation, the Bay of Bengal's near-surface layers are distinctly fresher than its deeper waters, thus strengthening and sharpening vertical density stratification (Lucas et al., 2016). The voluminous precipitation over the Bay and over surrounding river drainage basins, occurring mostly in the summer months, is a manifestation of the annual atmospheric southwesterly monsoon circulation, characterised by cross-equatorial surface winds transporting moisture across the Indian Ocean (Schott and McCreary Jr, 2001). The input of freshwater in the Bay also creates a rich horizontal structure in the buoyancy field of the surface layer (MacKinnon et al., 2016). Seasonal monsoon winds push buoyant surface layers around via Ekman transport (Sree Lekha et al., 2018), while energetic mesoscale eddies stir and strain them, creating long thin regions with large gradients (Hormann et al., 2016). These sharp density fronts give rise to submesoscale instabilities, further modifying the spreading, mixing, and stratification of freshwater (Sarkar et al., 2016).

Submesoscale dynamics include several different types of frontal instabilities. Some increase vertical velocities, and enhance fluxes between the surface and interior layers (Lévy et al., 2001), while others re-stratify surface mixed layers, isolating them from deeper layers (Mahadevan et al., 2012). Both are critical processes for ocean biological production. Submesoscale instabilities interacting with balanced geostrophic currents can transfer energy in a downscale cascade from the mesoscale to dissipative scales (Capet et al., 2008a), potentially a significant route to balance the ocean's energy budget. Of interest are the penetration and effects of submesoscale dynamics below the surface layer. Also of interest 


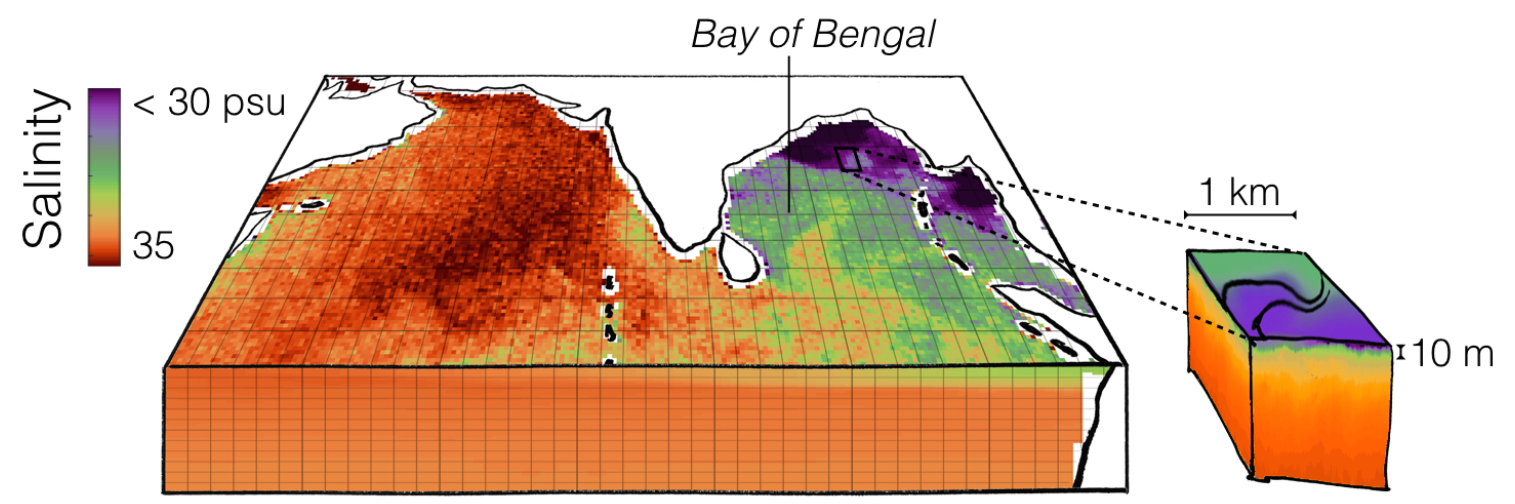

Figure 0-1: Illustration of salinity distribution in the northern Indian Ocean (based on a satellite-measured surface snapshot and a climatological sub-surface section). Near the surface, the Bay of Bengal is much fresher $(\lesssim 33 \mathrm{psu})$ than the neighbouring Arabian Sea $(\sim 36 \mathrm{psu})$ or the deeper interior water. It also contains very shallow $(\sim 10 \mathrm{~m})$ fresh layers with very sharp salinity fronts $(0.2 \mathrm{psu}$ across $10 \mathrm{~m})$.

are interactions of submesoscale frontal flows with oscillations of comparable scale, such as near inertial waves in the stratified upper ocean (Grisouard and Thomas, 2016; Nagai et al., 2015), as well as the stirring and distribution of tracers. At the surface, submesoscale vortices and flow structures contribute to lateral stirring and dispersion of flotsam or pollutants. Such flow fields remain unresolved by satellites or operational forecast models (Poje et al., 2014).

The distribution of freshwater and the dynamics of submesoscale phenomena are closely linked in the Bay of Bengal, and a theme of this thesis. The work presented in the following chapters was undertaken as part of a larger international project that brought together oceanographers from U.S., Indian, and Sri Lankan institutions to illuminate the characteristics and behaviour of the Bay of Bengal, especially with regards to interactions with the monsoon (Lucas et al., 2014). Supported on the U.S. side by the Office of Naval Research, the ASIRI (Air Sea Interactions Regional Initiative) and MISO-BOB (Monsoon Intra-Seasonal Oscillations - Bay of Bengal) projects, in collaboration with the Indian OMM (Ocean Mixing and Monsoon) initiative, conducted several field campaigns, starting in 2013 and with continuing efforts ongoing in 2019.

The project's motivation springs from the Bay of Bengal's potential role in shaping the Indian Summer Monsoon's pattern and timing of precipitation over the Indian subcontinent (Shenoi et al., 2002). With wide-ranging socioeconomic impact, monsoon rains are a tantalizing target of efforts to improve weather forecasts, especially medium range 
and seasonal predictions (Webster et al., 1998). The overarching goal of these efforts is to accurately reproduce and predict, in coupled atmosphere-ocean numerical models, the Bay of Bengal's SST, its boundary layer thickness, and its modulation of heat and moisture fluxes. To that end, accurate simulation of the effects of sub-surface dynamics on stratification is key, yet currently not well accomplished (Chowdary et al., 2016). It is unclear whether the problems lie in the vertical or horizontal resolution of models (Benshila et al., 2014), parametrizations neglecting complex turbulence structures (Shroyer et al., 2016a), faulty freshwater forcing at coastlines (Seo et al., 2009), or a combination of issues.

Driven by these unknowns, the research initiative has parallel goals of refining our knowledge of the region's unique characteristics and behaviour, while improving our general understanding of ocean dynamics, especially in the submesoscale. These goals were approached by exploring the Bay of Bengal's sub-surface hydrographic conditions and dynamic regimes with a variety of observational methods and systems (Wijesekera et al., 2016). Yet a significant challenge in this endeavour is making direct sub-surface observations of the flow field across the 0.1 - 10 kilometer lateral scales needed to resolve submesoscale phenomena, in a synoptic time frame. A further challenge is to isolate signals of submesoscale turbulence from wave oscillations (Callies and Ferrari, 2013).

This thesis offers an alternative and complimentary approach to investigating the dynamics in the Bay of Bengal, indirectly quantifying properties of the flow fields by looking at the thermohaline patterns created by the flows. Due to seawater density's dual dependence on temperature and salinity, thermohaline contrasts in the ocean can contain both a dynamically relevant component, i.e. buoyancy or density gradients, and a dynamically passive component, i.e. gradients of temperature exactly compensated in density by gradients of salinity (Stommel, 1962). Along surfaces of constant density, anomalies of salinity that are density-compensated by equivalent temperature anomalies, are called 'spice' anomalies, marking different 'water-masses' (Munk and Warren, 1981). Like patterns of paint that record the movements of a brush over canvas, thermohaline spice patterns record characteristics of the flows that stir the ocean's water. The Bay of Bengal provides a rich thermohaline field to study (Jaeger, 2018), with an annual surface supply of rain and river runoff, and subsurface injections of water originating from the saltier Arabian and fresher Andaman Seas (Gordon et al., 2016).

The following thesis chapters use the Bay of Bengal as a real-world fluids laboratory, 
essentially considering it a large water bucket containing two contrasting colored dyes, with which we test theories of geophysical fluid dynamics without scaling down. The observations and analyses illuminate both the region's special characteristics, and test ocean dynamical theories in a unique regime of very high stratification and small Coriolis parameters.
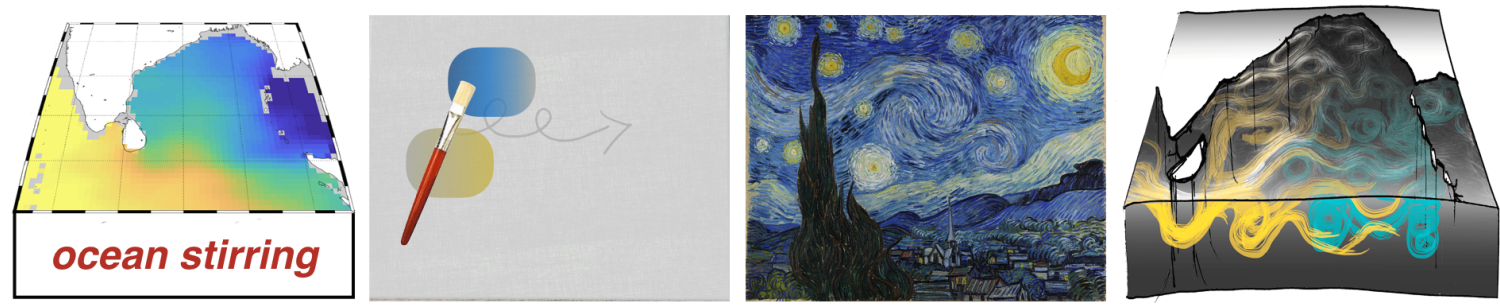

Figure 0-2: Like the lines of paint on the canvas of "The Starry Night" record the movement of the painter's brush (Vincent van Gogh, 1889), so the filaments of thermohaline spice reflect the stirring of ocean flows.

The chapters of this thesis are written to be largely self-contained, and can be read independently. A common thread is the pursuit of clues in the patterns of salinity and temperature to understand the dynamics. Often the dynamical regimes, identified with the help of freshwater anomalies, are also directly or indirectly influenced by the freshwater, originating in the monsoon's rainfall and associated river runoff.

For example, Chapter 1 discovers small-scale-only thermohaline compensation in the winter-time surface layer of the Bay of Bengal. Using a combination of sub-surface hydrographic data analysis and idealized numerical modeling of a salinity front, we show the temperature-salinity compensation to be the signature of submesoscale circulations, restratifying the surface mixed layer during winter time. These circulations are powered by the sharp salinity fronts at the edges of layers of freshwater that spread out from river runoff. The monsoon winds play an equally critical role in the compensation of re-stratified salinity fronts, strongly and persistently extracting heat from the ocean's surface layer, highlighting the frontal edges that contain this heat loss in the thinnest layer, thereby partially compensating the salinity-dominated fronts.

Similarly in Chapter 2, a surprising signature is discovered in a statistical analysis of spice patterns in the stratified interior of the Bay of Bengal, likely of submesoscale turbulence or shear dispersion. The scale-dependent variance distribution of a passive tracer, spice anomalies along surfaces of constant density, reveals much about the dynamical regimes in the upper $200 \mathrm{~m}$. The tracer gradient spectra support the modifications of quasigeostrophic 
theory, such as surface QG and frontogenesis, to be important at all but the largest scales and the deepest layers sampled. The distinct tracer variance slopes along isopycnals in close depth proximity $(\sim \Delta 50 \mathrm{~m})$, but large density separation $\left(\sim \Delta 2 \mathrm{~kg} \mathrm{~m}^{-3}\right)$, attests to the strong dynamical 'insulation' that the salinity stratification provides between surface layers and relatively shallow interior layers in the Bay of Bengal. Yet at the $40-70 \mathrm{~m}$ depths of strongest stratification, at the base of the surface mixed layer, the relative lack of thermohaline variance at scales of $1-10 \mathrm{~km}$ raises the possibility that submesoscale turbulence affects not only the surface mixed layer, but also the stratified layers right below. A change in spectral slope around $10 \mathrm{~km}$ scales is indicative of a change in the rate-limiting process transferring variance across scales. Seeing the unexpected behaviour in the spectra motivated the investigation of stirring mechanisms at submesoscale in the last chapter.

The dynamics that control and contribute to tracer stirring are further investigated in Chapter 3, prompted by both the surprising spectral results of Chapter 2, and striking hydrographic observations of tracer patterns in the previously analyzed sections. The relative lack of tracer variance below $10 \mathrm{~km}$ scales calls for one or more processes that are limited to stirring at and below these scales, since larger mesoscale eddies would not produce such a change in spectral slope. The observations offer clues in the form of tracer patterns of multiple, parallel, thin layers of alternating watermass characteristics. Analysing these two-dimensional patterns of spice, and spice curvature, in high-resolution upper ocean hydrographic sections, we find evidence in support of submesoscale eddies as well as near-inertial waves stirring the near-surface pycnocline. We propose two new formation mechanisms of sloping water mass features. Stirring by both submesoscale sheared eddies, or by highvertical-wavenumber near-inertial waves, can create thermohaline layering and interleaving with much longer-and-thinner aspect ratios than tracer features produced by mesoscale stirring. The shorter time scale of multiple layer production by these processes, with a greater cross-isopycnal surface area due to the thinner vertical scale of individual layers, suggests that either of these submesoscale or near-inertial mechanisms can enhance horizontal stirring, and potentially effective horizontal mixing, by shear dispersion.

The following three chapters paint a picture of the Bay of Bengal filled with submesoscale turbulence restratifying the surface layer and horizontally stirring and mixing below the surface. A conclusion and outlook is offered at the end. 


\section{Chapter 1}

\section{Submesoscale-Selective Compensation of Fronts in a Salinity-Stratified \\ Ocean}

\section{$1.1 \quad$ Abstract}

Salinity, rather than temperature, is the leading influence on density in some regions of the world's upper oceans. In the Bay of Bengal, heavy monsoonal rains and runoff generate strong salinity gradients that define density fronts and stratification in the upper $50 \mathrm{~m}$. Ship-based observations made in winter reveal that fronts exist over a wide range of length scales, but at $\mathrm{O}(1)$-km scales, horizontal salinity gradients are compensated by temperature to alleviate about half the cross-front density gradient. Using a process study ocean model, we show that scale-selective compensation occurs because of surface cooling. Submesoscale instabilities cause density fronts to slump, enhancing stratification along-front. Specifically for salinity fronts, the surface mixed layer (SML) shoals on the less saline side, correlating sea surface salinity (SSS) with SML depth at O(1)-km scales. When losing heat to the atmosphere, the shallower and less saline SML experiences a larger drop in temperature compared to the adjacent deeper SML on the salty side of the front, thus correlating sea surface temperature (SST) with SSS at the submesoscale. This compensation of submesoscale fronts

${ }^{*}$ Manuscript published in Science Advances, 28 Feb 2018, Vol. 4, no. 2, e1701504, DOI: 10.1126/sciadv.1701504; with 2nd author Amala Mahadevan. Copyright (C)2018 The Authors, some rights reserved. Distributed under a Creative Commons Attribution NonCommercial License 4.0 (CC BY-NC). 
can diminish their strength and thwart the forward cascade of energy to smaller scales. During winter, salinity fronts that are dynamically submesoscale experience larger temperature drops, appearing in satellite-derived SST as cold filaments. In freshwater-influenced regions, cold filaments can mark surface-trapped layers insulated from deeper nutrient-rich waters, unlike in other regions, where they indicate upwelling of nutrient-rich water and enhanced surface biological productivity.

\subsection{Introduction}

Lateral and vertical gradients in the upper ocean's density, namely, fronts and stratification, play an important role in upper ocean dynamics. Vertical stratification governs the depth and stability of the surface mixed layer (SML) and the behavior of internal waves and eddies. Fronts, or lateral density variations, give rise to baroclinic instability and eddies that convert potential energy to kinetic energy in the ocean, resulting in the conversion of horizontal density gradients to stratification. In regions that are nutrient-limiting for phytoplankton growth, fronts can enhance vertical supply of nutrients (Lévy et al., 2001), whereas in light-limited regions, frontal slumping can enhance stratification and light exposure for phytoplankton (Mahadevan et al., 2012). In much of the world's upper oceans, temperature variations govern the spatial gradients and seasonal variations of density, whereas salinity plays a secondary role (Johnson et al., 2012). Sea surface temperature (SST) derived from satellites is therefore an indicator of ocean dynamics and reveals fronts, filaments, and eddies at scales of $\mathrm{O}(1$ to 10$) \mathrm{km}$. In temperature-stratified regions, surface cooling destabilizes the density stratification and causes a deepening of the SML and entrainment of subsurface waters that are relatively rich in nutrients. In general, cold SST features are interpreted as upwelled or vertically mixed waters that are nutrient-rich and support phytoplankton productivity.

However, in regions that are strongly influenced by freshwater runoff and rain or have close-to-freezing surface temperatures, salinity variations can be the leading control on nearsurface density gradients (Timmermans and Winsor, 2013; MacKinnon et al., 2016). In these regions, the near-surface layer's response to atmospheric cooling differs from regions where the density of the SML is temperature-controlled. Here, density stratification is controlled by salinity, and the depth of the SML is defined by the vertical salinity gradient, so temperature 
can remain uniform (or even increase) with depth beneath the SML in the so-called barrier layer. By losing heat to the atmosphere, the SML can become colder than layers that lie subsurface, while still remaining stably stratified because of salinity (Mahadevan et al., 2016). Areas of the SML that cool the most by heat loss to the atmosphere are those with the shallowest and strongest salinity stratification.

Although lateral density gradients or fronts occur over a wide range of scales, kilometerscale fronts within the SML of the ocean form submesoscale instabilities and mixed-layer eddies that promote the downscale cascade of energy (Capet et al., 2008a) while converting lateral buoyancy gradients into mixed-layer stratification. Such a process of restratification of the mixed layer by submesoscale baroclinic instability has been recently modeled and observed in a number of contexts (Fox-Kemper et al., 2008; Mahadevan et al., 2012). Because of the slumping of horizontal density gradients, the shallowest and strongest stratification forms along the edges of eddies and frontal instabilities, which also exhibit the strongest horizontal density gradients. The density stratification arising from salinity-controlled fronts is also salinity-controlled and not convectively unstable when cooled from above. Thus, when cooled by the atmosphere, the restratifying frontal regions experience a greater drop in SST than their surroundings (Fig. 1-15). This delineates them as cold filaments in satellite imagery. These cold filaments in salinity-controlled regions differ crucially from those in temperature-controlled regions. They emerge along submesoscale fronts where restratification by slumping cuts off communication with the subsurface.

An important facet of salinity-controlled density fronts in the SML is that they become temperature-compensated by atmospheric cooling where they restratify (Fig. 1-15). This occurs at kilometer scales where lateral density gradients are dynamically unstable within the SML and submesoscale in character. In what follows, we use high-resolution measurements of upper ocean temperature and salinity from the Bay of Bengal (BoB) in the Indian Ocean to reveal the scale-selective compensation of fronts that is observed in winter. We then use a process study model to demonstrate the underlying mechanism for the observed phenomenon. Finally, we discuss the prevalence and implications of the phenomenon. 


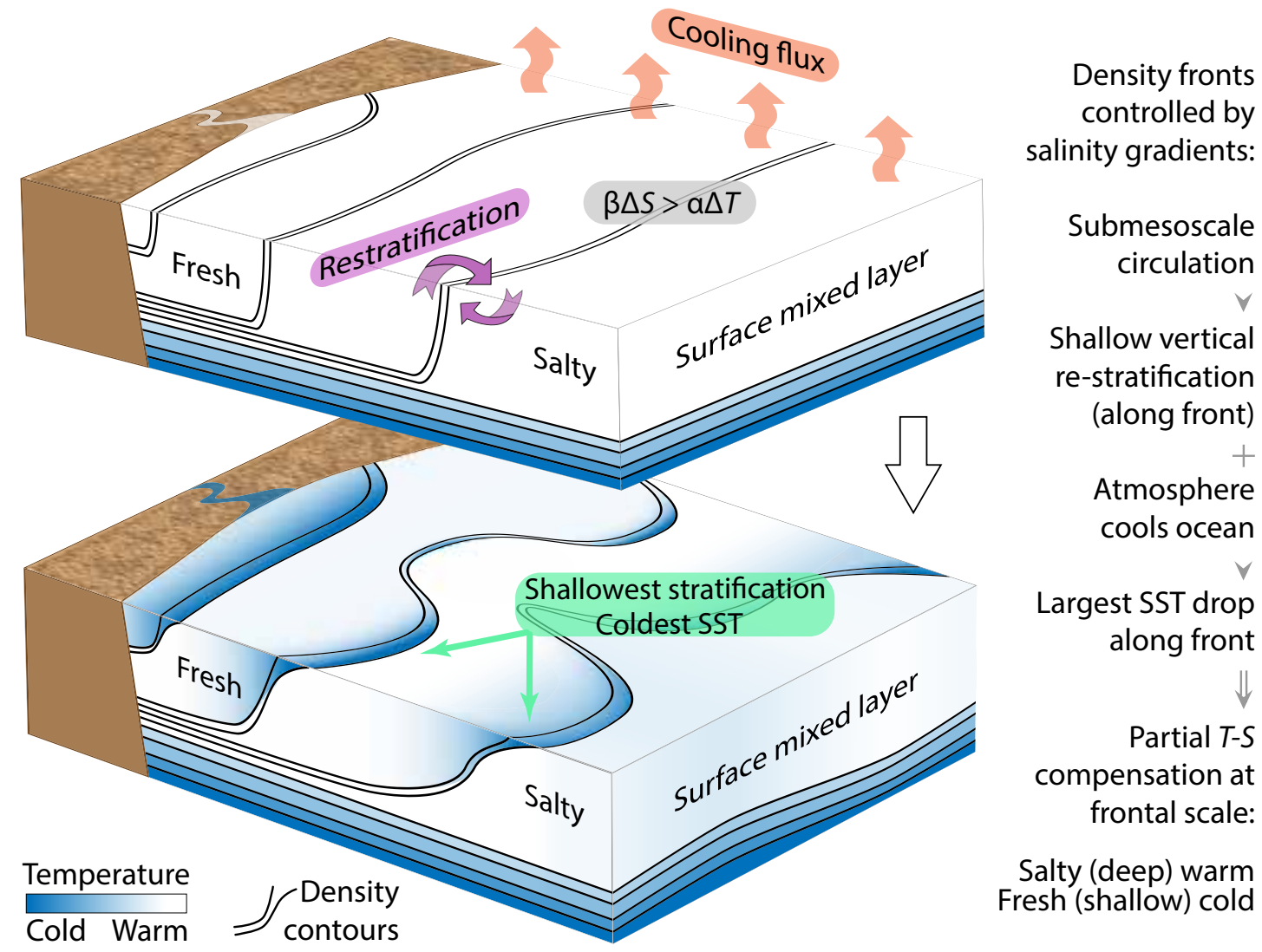

Figure 1-1: Schematic drawing of the mechanism by which cooling creates densitycompensated submesoscale fronts and cold SST filaments. In a salinity-stratified upper ocean, submesoscale instabilities of salinity fronts cause restratification and shallowing of the SML. Cooling is concentrated within shallow (most stratified) SMLs, creating a larger drop in SST along salinity-stratified fronts, at scales of submesoscale restratification. SST and SSS become correlated (salty warm and fresh cold, compensating their effects on density) at length scales $\mathrm{O}(1) \mathrm{km}$.

\subsection{Materials and Methods}

\subsubsection{Remote Observations}

The $\mathrm{BoB}$ is an exceptionally fresh, tropical ocean, receiving a large (circa $1.5 \mathrm{~m} /$ year) net input of fresh water from monsoonal rains and runoff (Rao and Sivakumar, 2003; Shetye et al., 1996). Although the surface waters are warm $\left(\sim 25^{\circ}\right.$ to $30^{\circ} \mathrm{C}$ on average $)$, the depth and dynamics of the SML are strongly controlled by salinity (Sengupta et al., 2016). Large riverine inputs along the northern margins of the basin result in strong lateral salinity gradients that form density fronts within the SML (Mahadevan et al., 2016). The fresher water, being less dense, forms a shallow SML with the vertical density gradient beneath the 
SML defined by the gradient in salinity (Girishkumar et al., 2013). In many areas, we can observe a barrier layer, or layer between the halocline (which defines the depth of the SML) and thermocline (which defines the base of the isothermal layer) (Thangaprakash et al., 2016).

Contrasting behaviors can be seen in the Arabian Sea (AS) and BoB, the two northernmost regions of the Indian Ocean that flank the Indian subcontinent to the west and east. By early December, the seasonal surface winds over the northern Indian Ocean blow steadily from the northeast, advecting relatively dry and cool continental air over the AS and the BoB. This leads to a loss of heat and moisture to the atmosphere, cooling the sea surface [based on NASA Aquarius data (Fig. 1-2) (Lagerloef et al., 2012); NASA MODIS (Moderate Resolution Imaging Spectroradiometer) data (Fig. 1-2, B and D); NCEP GODAS (National Centers for Environmental Prediction Global Ocean Data Assimilation System) reanalysis (Fig. 1-2C) (Kalnay et al., 1996)]. Although SSTs in these two embayments span a similar range $\left(24^{\circ}\right.$ to $\left.29^{\circ} \mathrm{C}\right)$ at this time, their sea surface salinities (SSSs) are very different. SSS in the AS is between 36 and 37 practical salinity units (psu), whereas in the fresher BoB, it varies between 28 and 34 psu. Taking into account the relative magnitudes of the thermal expansion and haline contraction coefficients, these SST and SSS fields show that horizontal density gradients within the SML are controlled by temperature in the AS but by salinity in the BoB.

In the AS, the cold SST is correlated with high chlorophyll in the ocean color imagery from a satellite, which is indicative of vertical mixing or upwelling of nutrients from the subsurface. Entrainment of deeper colder (denser) waters into the SML lowers the SST and also leads to higher biological productivity in the AS (Kumar et al., 2001).

A similar cold and biologically productive patch is seen in the BoB off the east coast of India because of vigorous mixing caused by the cyclonic storm Madi, which was active between December 6th and 13th (track marked in Fig. 1-2C). However, the cold SST features in the open bay north of $16^{\circ} \mathrm{N}$ lack any chlorophyll signature. These low SST regions in the northern $\mathrm{BoB}$ are not denser than their surroundings and are not formed because of vertical mixing or upwelling. The cooler water near surface is held up by salinity stratification as will be shown from in situ data (Fig. 1-2B). Whereas the scale of these cold SST patches and filaments is barely resolved by Aquarius SSS, it is better resolved by the newly available $1 / 4^{\circ}$ Soil Moisture Active Passive satellite-derived SSS maps. In a 

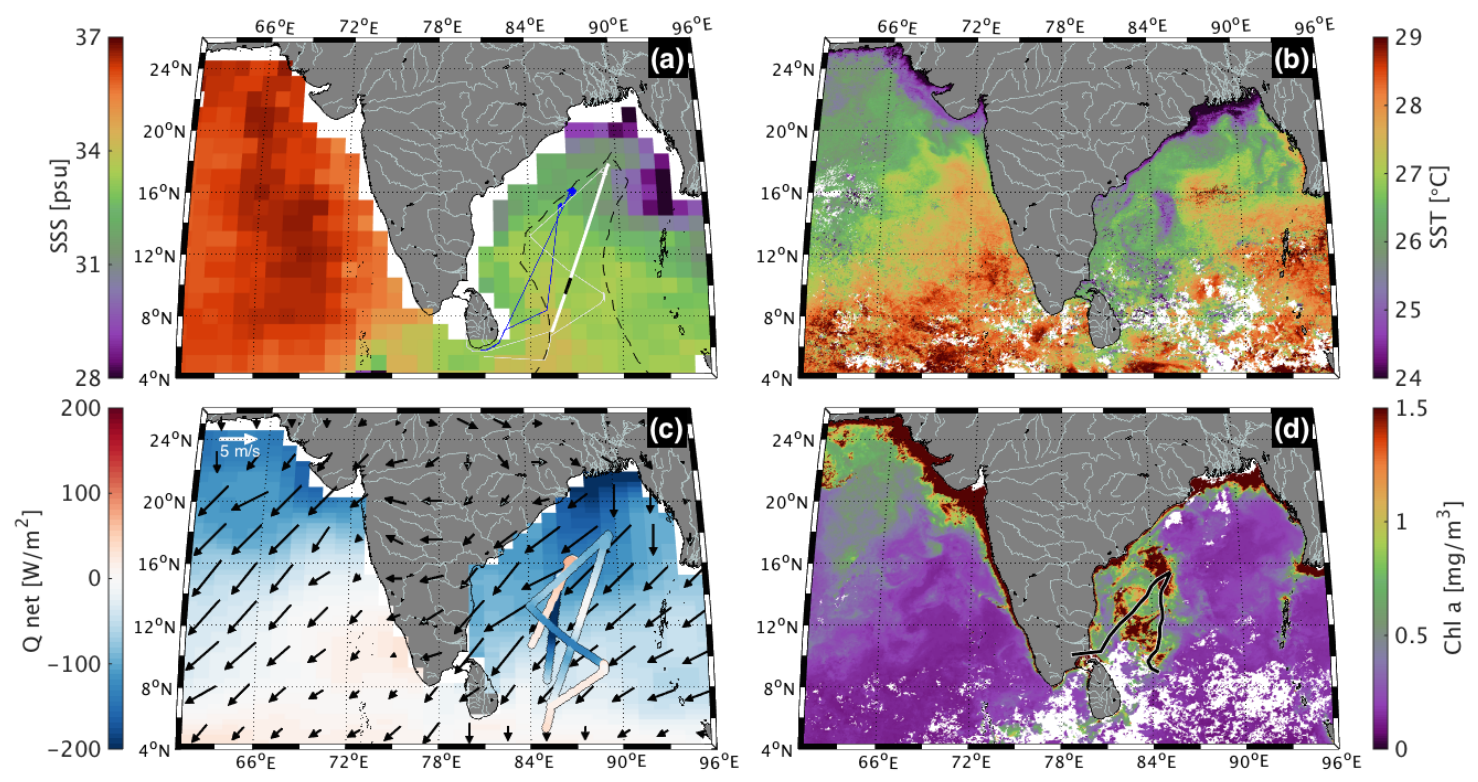

Figure 1-2: Winter-monsoon sea surface conditions in the northern Indian Ocean (west, AS; east, BoB). (A) Surface salinity (November to December 2013, Aquarius satellite data). Thin lines mark the ship's track from 10 to 26 November (blue) and from 28 November to 13 December (white). Thick segments correspond to sections shown in Fig. 1-3. The dashed line marks the limits of international waters. (B) Nighttime SST (11 to 18 December, MODIS). (C) Net heat flux and surface winds (November to December 2013, NCEP reanalysis). Colored track show net daily heat flux measured during the surveys. (D) Chlorophyll $a$ (Chl a) (25 November to 26 December, MODIS); track of the cyclonic storm Madi (6 to 13 December, Joint Typhoon Warning Center) shown in black. Dates of satellite data composites are chosen to provide a cloud-free view during the ship survey period.

representative snapshot of the northern BoB during the winter of 2015, we find that cold and fresh patches are generally colocated (Fig. S1). In addition to the coldest water found at the northern coastline where the fresh river discharge enters the bay, cold filaments also appear along the front where the freshest water meets saltier water in the eastern BoB.

Further insight into the relationship between salinity $(S)$ and temperature $(T)$ is provided by a detailed view of the hydrography obtained by conducting a high-resolution survey of the BoB from the $R / V$ Roger Revelle in 2013.

\subsubsection{Ship-based Observations}

Surface and subsurface salinity and temperature fields during the Northeast Monsoon were measured in two surveys between 10 to 26 November and 29 November to 11 December 2013. The surveys accomplished six transects between $5^{\circ}$ and $18^{\circ} \mathrm{N}$, the longest of which was about $1200 \mathrm{~km}$ long (Fig. 1-2). Underway conductivity-temperature-depth (UCTD) 
profiles taken at fairly regular intervals (averaging $5 \mathrm{~km}$ in separation during the straight transects of the second survey) show a fresh near-surface layer above a strong halocline that lies between 30- and 50-m depths. Temperature is generally inverted within this surface layer, but the vertical density gradient is kept stable by salinity stratification. Fig. 1-3 shows a 100-km-long section of temperature, salinity, and density stratification (characterized by buoyancy frequency $N^{2} \equiv \frac{-g}{\rho_{o}} \frac{\partial \rho}{\partial z}$, where $\rho$ is the density, $\rho_{o}$ is the reference density, and $g$ is the acceleration due to gravity). The observed temperature inversion can be ascribed to the net air-sea heat flux of $-50 \mathrm{~W} / \mathrm{m}^{2}$ (mean) computed from bulk meteorological measurements during the survey. The net flux is the sum of latent $\left(-120 \mathrm{~W} / \mathrm{m}^{2}\right)$, longwave $\left(-50 \mathrm{~W} / \mathrm{m}^{2}\right)$, and sensible $\left(-10 \mathrm{~W} / \mathrm{m}^{2}\right)$ mean negative components that exceed the mean solar shortwave $\left(+130 \mathrm{~W} / \mathrm{m}^{2}\right)$ warming flux. The dominant latent and shortwave components varied with wind speed, humidity, and cloud cover variations, whereas the variability of SST being less than $1^{\circ} \mathrm{C}$ had a comparatively small effect. Because the time scale and length scale of these atmospheric patterns are fast and large compared to submesoscale ocean processes (a mean wind speed of $7 \mathrm{~m} / \mathrm{s}$ covers $600 \mathrm{~km}$ in a day), the net heat flux is considered uniform for our analysis.

Although the spacing of UCTD profiles allowed fronts wider than $\mathrm{O}(10) \mathrm{km}$ to be resolved, the ship's flow-through thermosalinograph (TSG) system measured the $T$ and $S$ of water continuously pumped from a hull intake every $15 \mathrm{~s}$. Because of the ship's displacement and disturbance of the surrounding water, these measurements are representative of the top $5 \mathrm{~m}$ of the ocean, which lies above the mean SML depth as diagnosed from the UCTD profiles. At a cruising speed of 11 knots, this sampling corresponds to a resolution of about $0.1 \mathrm{~km}$. The TSG thus fully resolves length scales of $\mathrm{O}(1) \mathrm{km}$, enabling us to observe submesoscale (kilometer-scale) horizontal gradients and features in the SML (Fig. 1-3).

Horizontal gradients of density (or buoyancy) within the SML are dominated by $S$, whereas $T$ is nearly uniform. The data in Fig. 1-3A are plotted such that equal vertical excursions along the $T$ and $S$ axes represent equal changes in density, albeit of opposite sign, as in the study of Rudnick and Ferrari (Rudnick and Ferrari, 1999). Although horizontal density-scaled temperature gradients in the SML are negligible compared to salinity gradients at large scales, they become comparable at scales of a few kilometers, with temperature partially compensating salinity's control of lateral density variations at scales of $\mathrm{O}(1) \mathrm{km}$. This pattern of $T$ - $S$ coherence at small scales is seen not only in this highlighted section but 

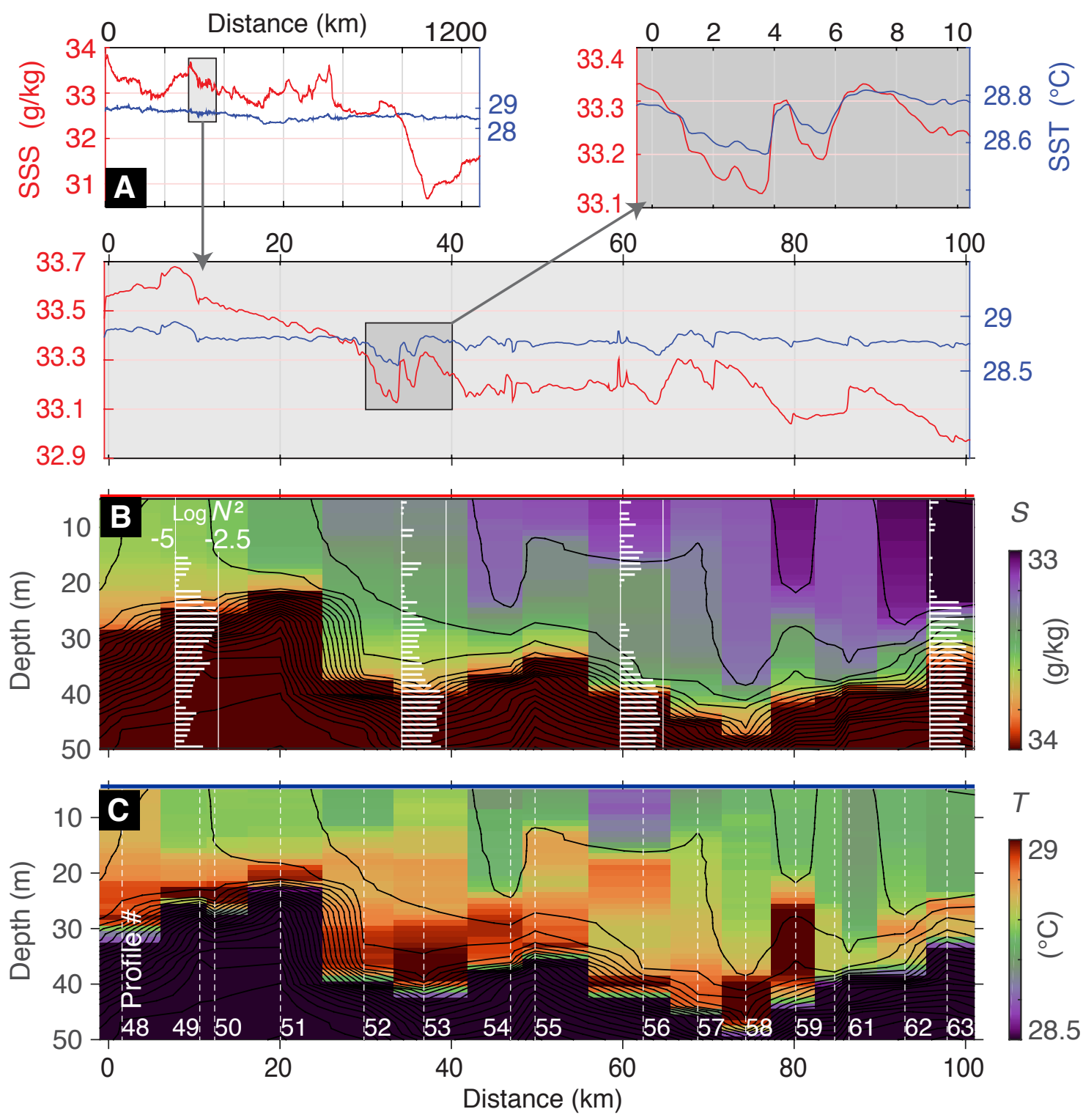

Figure 1-3: Surface-layer salinity and temperature structure in the BoB observed between 29 November and 3 December 2013. (A) $1200 \mathrm{~km}$ south-to-north transect (marked on map) of surface salinity (red) and temperature (blue) from a shipboard TSG. Shaded boxes show a 100- and 10-km section in detail. The ranges of vertical $T$ and $S$ axes are proportionally scaled by the thermal expansion and haline contraction coefficients $\alpha$ and $\beta$, such that graphically equal displacements have equal effect on density. Compensating correlation is seen at scales below $\mathrm{O}(10) \mathrm{km}$. (B) Salinity and (C) temperature section composed of UCTD profiles (numbered, dashed vertical lines) taken during the 100-km section plotted above. Density (in black contours) and a few profiles of stratification ( $\log N^{2}$ indicated by white bar graphs) are overlaid. Note the cooler surface waters confined above warmer subsurface layers by stable salinity stratification. 
throughout the entire survey.

\subsection{Analysis}

To quantify the relative importance of the horizontal temperature and salinity gradients on the horizontal density gradients (frontal intensity), we calculate the density ratio, $R=$ $\alpha \Delta T / \beta \Delta S$, and related Turner angle $(T u=\arctan R)$ (Ruddick, 1983), where $\Delta T$ and $\Delta S$ are the horizontal variations of $T$ and $S$ between two points along track and $\alpha$ and $\beta$ are the thermal and haline expansion coefficients calculated at the measured $T$ and $S .|R|<1$ indicates that salinity gradients are more important than temperature gradients in setting the density gradient, and the opposite is true for $|R|>1$. Positive $R$ indicates compensating $T$ and $S$ effects on density. At $R=1$, equal and opposite $T$ and $S$ gradients cancel out any density variation. The mapping of $R$ onto $T u$ is chosen such that the origins coincide and Tu ranges between $\pm \pi / 2$ (Fig. 1-4). Following the study of Rudnick and Martin (Rudnick and Martin, 2002a), our analysis is performed with $T u$ because $T u$, unlike $R$, is confined to a finite range, but the results are described using the corresponding, and more intuitive, $R$ values.

For each of the six survey sections, we perform a wavelet decomposition of $T$ and $S$ to separate the gradients by spatial scale as in the study of Rudnick and Ferrari (Rudnick and Ferrari, 1999), which is in effect similar to a series of band-pass filters. At each level (scale) of the $T$ and $S$ decomposition, finite differences between adjacent wavelet coefficients are used to calculate $R$, yielding a gradient scale-specific distribution of density ratios (more details available in the Supplementary Materials). The histograms of $R$ at 100-, 10-, and 1-km scales (black curves in Fig. 1-4A) from the 1200-km-long section shown in Fig. 1-3A reveal a narrow peak around $R=0$ at the large $(\sim 100 \mathrm{~km})$ scale. However, the peak of the distribution is shifted toward positive $R$ at 10-km scales and even more so at 1-km scales. At this smallest resolved scale, the strongest fronts are salinity-controlled and partially compensated by temperature so that $R$ lies between 0 and 1 with a median of 0.3 .

The distributions of $R$ include segments where both $T$ and $S$ gradients happen to be negligibly small (Fig. S2), yielding some very large magnitudes of $R$ that make the distributions long-tailed (black curves in Fig. 1-4A). To highlight just the strongest $T$ and $S$ fronts, we select for the largest $5 \%$ of individual $\alpha \Delta T$ and $\beta \Delta S$ gradients shown as discrete 

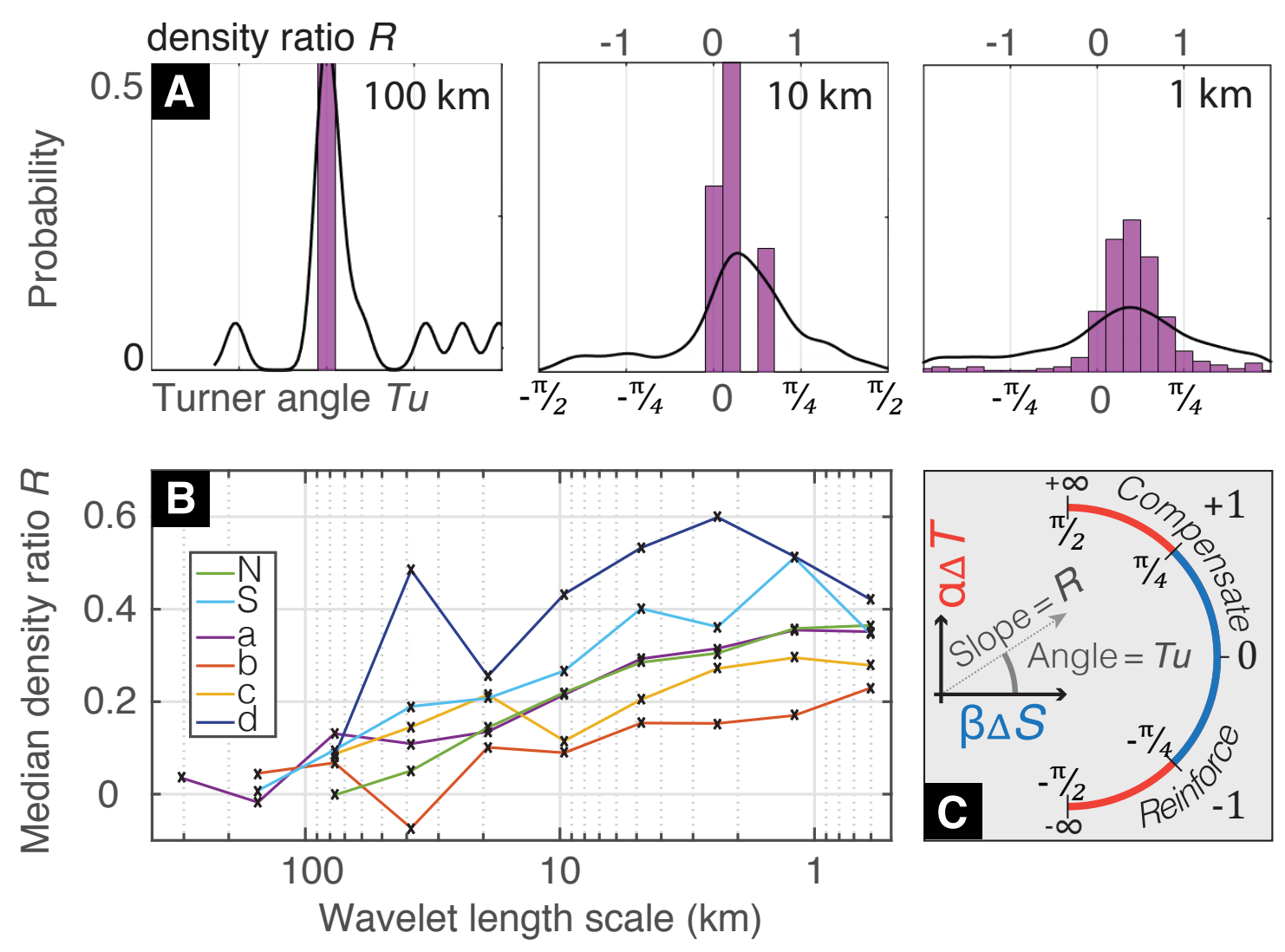

Figure 1-4: Scale dependence of T-S compensation. (A) Probability distributions of $R$ (density ratio) for different horizontal gradient scales, 100, 10, and $1 \mathrm{~km}$, as estimated from the longest ship section (leg 2, section A in previous figures). Black lines show the distribution of $R$ for all gradients, whereas purple bars denote the distribution of $R$ for only the largest gradients (selected as the 95th percentile of individual $\alpha \Delta T$ or $\beta \Delta S$ magnitudes shown in Fig.S2). Density compensation of fronts is greater at $10-\mathrm{km}$ scales than 1000 $\mathrm{km}$ scales and greater at $1-\mathrm{km}$ scales than $10-\mathrm{km}$ scales $(R=1$ and $T u=\pi / 4$ denote perfect compensation). (B) Median of $R$ distribution as a function of horizontal length scale for each of the six ship transects. Pairs of $\alpha \Delta T$ and $\beta \Delta S$ are computed from a wavelet decomposition of the individual ship transects mapped in Fig. 1-2A. Lines $(\mathrm{N})$ and $(\mathrm{S})$ are from the earlier cruise, and lines (a) to (d) are from the later cruise, both during November to December 2013. All transects are consistent in showing that greater density compensation is favored at smaller scales. (C) Schematic demonstrating the relationship between density ratio $R=\alpha \Delta T / \beta \Delta S$ and Turner angle $T u$.

bars in the histogram (Fig. 1-4A). These agree with the distributions that include both strong and weak fronts (black lines) but show an even narrower range of $R$ confined between 0 and 1.

All ship transects are consistent in showing that greater density compensation is favored at smaller scales of $\mathrm{O}(1) \mathrm{km}$. The medians of the distributions are near 0 at large $\mathrm{O}(100)$ - 
$\mathrm{km}$ scales but positive (between 0 and 1 ) at smaller scales. In other words, $T$ gradients are negligible at large scales but increasingly compensate $S$ gradients at the submesoscale. The transect showing the greatest compensation at all scales (leg 2, transect D) is anomalous because it was taken after the cyclonic storm Madi passed over the southern BoB in early December.

A previous study of the $T$-S relationship (Ferrari and Rudnick, 2000) in the subtropical Pacific found that the $T$ and $S$ gradients in the SML compensate each other at all scales ranging from $1000 \mathrm{~km}$ to $10 \mathrm{~m}$. Distributions of density ratios, calculated at scales of 10 , $1,0.1$, and $0.02 \mathrm{~km}$, were all centered around $R=1$, independent of the length scale. The authors' explanation for the scale-independent predominance of compensation is that although stochastic forcing can create $T$ and $S$ gradients with a broad distribution of density ratios at the scale of the surface buoyancy forcing, any uncompensated $T$ or $S$ gradient $(R \neq 1)$ is a density front, which is dynamically active and is dissipated or mixed away. However, a compensated $T$-S gradient ( $R=1$ ) has no density signature (although it has a gradient in the passive tracer "spice") and is thus not dynamically active. Instead, it can be stirred by eddies to finer and finer scales, while maintaining the same $R(=1)$ as the larger-scale gradient from which it originated. Other studies have continued to examine $T$ - $S$ compensation in open-ocean settings, finding compensation on 3 to $4 \mathrm{~km}$ scales in all oceans when the SML is deep but not when the SML is shallow (Rudnick and Martin, 2002a). Both compensated and uncompensated gradients are found at scales below $10 \mathrm{~km}$ in the North Pacific Subtropical Front (Hosegood et al., 2006).

However, the observation in the BoB of an abundance of partially compensated salinitydominated fronts $(0<R<1)$ and a relative absence of temperature-dominated fronts partially compensated by salinity $(R>1)$ is not explained by stochastic forcing. The scale dependence of the observed correlation cannot be ascribed to the downscale cascade of largerscale compensation by stirring because, here, the larger-scale gradients are uncompensated and compensation increases with decreasing length scales both from 100 to $10 \mathrm{~km}$ and from 10 to $1 \mathrm{~km}$. Furthermore, although density (salinity) fronts are observed at all scales, they are either uncompensated or partially compensated, but none of the strongest fronts are reinforced by temperature gradients acting in concert with the salinity gradient $(R<0)$.

We propose that the observed increased correlation of $T$ with $S$ at kilometer scales is the result of surface cooling of salinity gradient-dominated fronts (Fig. S3). Mesoscale 
eddying processes of $\mathrm{O}(100) \mathrm{km}$ stir and strain the large-scale salinity (density) gradients, sharpening fronts. Submesoscale instabilities acting on $\mathrm{O}$ (1 to 10) $\mathrm{km}$ scales (Thomas et al., 2008a) tend to slump these fronts through $\mathrm{O}(1)$ Rossby number processes, increasing shallow stratification beneath the lighter side of the front (Fox-Kemper et al., 2008). Near-surface stratification is then positively correlated with the surface buoyancy at this scale, with increased density stratification occurring where the surface salinity is low. This means that the depth of the SML is also correlated with salinity gradients at kilometer scales. If the surface is now cooled by an air-sea heat flux that is smooth (uniform) over kilometer scales and the surface heat loss is distributed into the mixed layer, then the shallower and fresher side has a smaller heat capacity and will incur a greater drop in temperature than the deeper and saltier side. This forcing mechanism would tend to correlate $T$ with $S$, moving $R$ from 0 to 1 at the scale of frontal restratification.

This compensation mechanism is scale-selective for two reasons. First, at scales larger than the submesoscale, there are many competing processes affecting the depth of the SML that are unrelated to local horizontal surface density gradients. Thus, large-scale patterns of SML depths are not well correlated with SSS, and cooling does not necessarily lead to large-scale compensation of SST with SSS. Second, for a given heat flux, the largest SST response is achieved in the shallowest SMLs found at the sharpest fronts. Thus, even if both the large-scale SML-depth variations and the small-scale restratified SML-depth variations are correlated with SSS, density compensation by temperature will be first achieved at the sites of shallow stratification, which are submesoscale fronts.

\subsubsection{Three-dimensional Modeling}

To demonstrate this mechanism creating scale-selective $T$ - $S$ compensation, we configured a nonhydrostatic, submesoscale permitting Process Study Ocean Model (PSOM) (Mahadevan et al., 1996) within a periodic channel $48 \mathrm{~km}$ (along front) $\times 96 \mathrm{~km}$ (across front) to simulate a salinity front in the SML (upper $40 \mathrm{~m}$ ), set above a pycnocline (Fig. 1-5). The front was initialized with a tanh cross-front salinity variation, connecting two vertical $S$ profiles measured during the same BoB cruise as the data in Fig. 1-3. Temperature $T$ was initialized from the same profile but is horizontally uniform. This created an idealized, uncompensated

salinity front, with realistic horizontal density gradients and vertical stratification. The model grid dimensions were $250 \mathrm{~m}$ in the horizontal (with $192 \times 384$ grid cells) and $1 \mathrm{~m}$ in 
the vertical near surface increasing with depth (64 levels). The numerical model is integrated with 1-min time steps. Subgrid processes were parameterized with a constant horizontal diffusivity of $0.2 \mathrm{~m}^{2} / \mathrm{s}$ and the $\kappa-\epsilon$ turbulence closure scheme (Burchard and Bolding, 2001), as previously described (Mukherjee et al., 2016). Additional model simulations were run with a 5- and 25-fold increase in horizontal diffusivity to 1 and $5 \mathrm{~m}^{2} / \mathrm{s}$, which lies within the observed range (Shcherbina et al., 2015). The model was also run with half the horizontal grid resolution, but the results were insensitive to these parameter changes (Figs. S4 and S5).

The density front was initially in thermal wind balance with a frontal jet, which became unstable and developed meanders and filaments within 1 week of model spin-up in which no surface forcing was applied. For the following week, the model was forced with a constant and uniform surface heat flux of $-50 \mathrm{~W} \mathrm{~m}^{-2}$ that cooled the ocean. In addition to the $\kappa-\epsilon$ scheme, a convective mixing scheme was used to rapidly distribute the buoyancy loss downward from the surface until static stability was achieved in the model.

\subsection{Model Results}

The initially uniform SST field develops kilometer-scale features correlated with SSS gradients of $\mathrm{O}(1$ to 10$) \mathrm{km}$. The largest drop in SST is found along the sharpest fronts, which in time, become cold filaments in SST. These cold filaments are not dense filaments connected to deeper colder waters, but rather, they mark an increased near-surface stratification forming extremely shallow SMLs on the less dense side along fronts. A vertical section through the model domain shows heat trapped beneath the cold fresh side of fronts; the temperature inversion is kept stable by the strong salinity stratification (Fig. 1-5C). On the domain scale, the differences in initial SML depths between the fresher side $(25 \mathrm{~m})$ and the saltier side $(50 \mathrm{~m})$ lead to a large-scale correlation (albeit weaker than the small-scale correlation) of SST with SSS because the uniform heat loss is confined to a shallower versus a deeper SML. A control run of the model without surface cooling develops similar salinity fronts and kilometer-scale features, but the salinity fronts remain uncompensated because the surface temperature remains uniform (Fig. S6).

We repeated our scale-selective analysis of density ratio distributions on a series of crosschannel surface transects from the model output (Fig. 1-5B). Within 1 day of cooling, the 


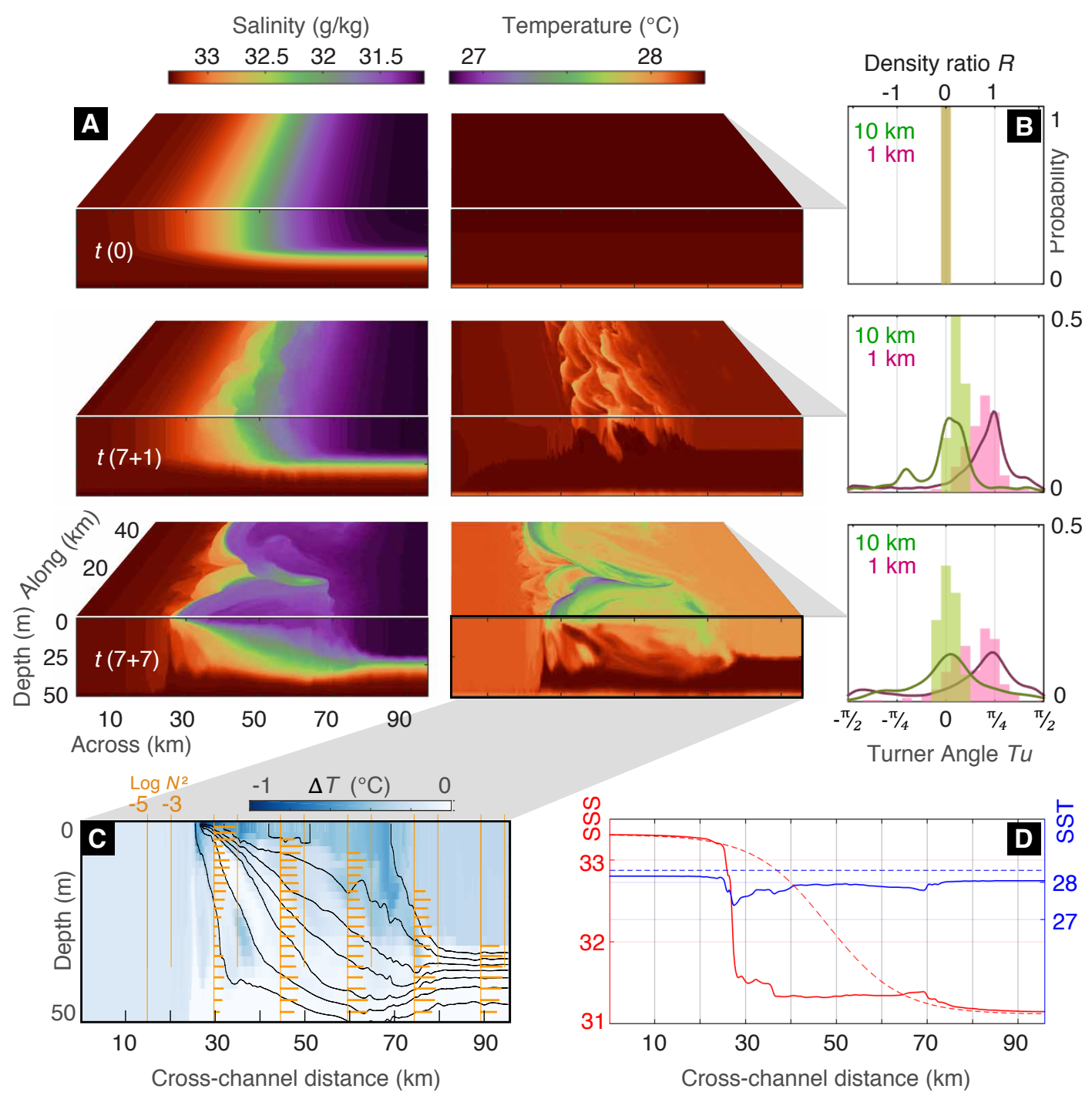

Figure 1-5: Numerical simulation of a submesoscale salinity front forced by surface cooling, developing O(1)-km scale T-S compensation. (A) Surface- and depth-dependent salinity (left) and temperature (right) from our process study numerical ocean model, or PSOM, configured in a periodic channel. The model domain extends 10 times deeper than what is shown. Top: Model initial condition at $t=0$ days. Middle: $t=8$ days, comprising 7 days of model integration without any heat flux and 1 day of cooling by a constant and uniform surface heat flux of $-50 \mathrm{~W} / \mathrm{m} 2$. Bottom: $t=14$ days of which the last 7 days are with cooling. (B) Distributions of surface density ratio $R$, initially (top), after 1 day of cooling (middle), and after 1 week of cooling (bottom). Filtering for horizontal gradient scales of order $10 \mathrm{~km}$ (pale green) and $1 \mathrm{~km}$ (pink). Lines show distributions for ratios of all gradients, whereas shaded bars show distributions for ratios of only the largest $T$ and $S$ gradients, as in Fig. 1-4. (C) Temperature change (of a cross-channel section) after 1 week of surface cooling. Isopycnals (black contours) and stratification (yellow bars). (D) SSS (red) and SST (blue) across the section initially (dashed line) and after the front evolves and is cooled for 1 week (solid line). 
initially uniform density ratio $(R=0)$ at both the 10 - and 1-km scales develops into a broader distribution of $R$ shifted toward positive values. After 1 week of cooling, the median $R$ value of strong fronts in the model is equal to 0.57 [for $\mathrm{O}(1) \mathrm{km}$ scales] and 0.06 [for $\mathrm{O}(10) \mathrm{km}$ scales], compared to 0.31 and 0.16 , respectively, in the TSG data. The interquartile range of the $\mathrm{O}(1) \mathrm{km}$ strong fronts falls between $R=0.19$ and 0.86 in the model and $R=0.15$ and 0.52 in the TSG data. Similar to the $T-S$ compensation observed in the BoB, the model exhibits compensation preferentially at smaller scales.

\subsection{Discussion}

Besides horizontal compensation, the greater temperature drop along the front also brings fresher water to the same density as slightly saltier but warmer water. Any along-isopycnal subduction of water at the front then increases the salinity variance along isopycnals, and isopycnal stirring and mixing can disperse the fresh water into saltier deeper layers. Simply put, the increased heat loss (buoyancy loss) of fresher water, compared to deeper saltier SMLs, decreases the density difference between fresh and salty water masses.

\subsubsection{Geographic Occurrence}

The prevalence of such cooling-induced compensation can be estimated globally by identifying regions in which both necessary factors, a cooling flux and salinity-dominated gradients, are simultaneously met in the SML. Using a climatology of surface $Q_{n e t}$ from OAFlux (Yu et al., 2008) and a climatology of horizontal density ratios, calculated from smoothed $T-S$ gradients in the SML based on Monthly Isopycnal/Mixed-layer Ocean Climatology (Johnson et al., 2012), a database of global SML salinity and temperature objective maps, we estimate the likelihood of these conditions being met in the ocean for each month of the year, placing greater weight on larger (negative) fluxes and stronger salinity control (smaller density ratio). For each $1^{\circ} \tilde{\mathrm{A}} \mathrm{U}^{\circ} 1^{\circ}$ ocean pixel, we calculate two normalized factors, $q=\frac{-Q_{\text {net }}}{50 \mathrm{~W} / \mathrm{m}^{2}}$ and $r=\frac{45^{\circ}-|T u|}{45^{\circ}}$, assigning 0 to negative values and limiting positive values to 1 . The product $\Gamma(x, y, t)=q \cdot r$ is an estimate for the occurrence of cooling-induced compensation for each month. The maximum value across all months is mapped in Fig. 1-6, along with the respective month of occurrence. Besides confirming the BoB's favorable conditions, these factors predict the mechanism to potentially be active in the polar oceans and coastal seas, espe- 
cially at higher latitudes, covering $20 \%$ of the global ocean (where $\Gamma>0.5$ in any month). These factors are based on climatological monthly mean fluxes. However, shorter periods of intense cooling can also compensate SSS gradients, and hence, this analysis provides a conservative estimate. Enhanced $T-S$ correlation at small scales is expected in regimes where large-scale salinity gradients create density fronts and the atmosphere cools the surface (such as coastal oceans with an active input of fresh water during fall and winter or polar seas). In regions of weak salinity gradients, such as the subtropical open oceans, this mechanism is not supported, and $T-S$ compensation is not expected to be enhanced at kilometer scales.

\subsubsection{Compensation Time Scale}

Dynamics at the submesoscale are thought to provide a crucial link between the large-scale energy input to the ocean and the scales of turbulent dissipation of energy (McWilliams, 2017). Whereas geostrophic turbulence causes energy to cascade to larger scales, ageostrophic frontal dynamics can convert baroclinic potential energy to kinetic energy at smaller scales. The kilometer-scale horizontal density gradients powering these circulations are controlled by $T$ and S. Here, we show that submesoscale-selective density compensation can weaken these fronts, forestalling the release of submesoscale energy to smaller scales through which dissipation can occur.

As a measure of the mechanism's importance in a given regime, we estimate the nondimensional density ratio $\left(R=\frac{\alpha \Delta T}{\beta \Delta S}\right)$ attained within the dynamical time scale of an inertial period $(2 \pi / f)$ by an initially uncompensated $(R=0)$ salinity front $(\Delta S)$ forced by a timemean cooling flux $(Q)$. If the restratified SML depth $\left(z_{f}\right)$ is less than the SML depth on the salty side $\left(z_{s}\right)$, the front changes more rapidly in temperature $\left(d T=\frac{-Q \cdot 2 \pi / f}{z \cdot c_{p} \cdot \rho_{o}}\right.$ where $c_{p}$ and $\rho_{o}$ are the specific heat capacity and the mean density of seawater, respectively, and $z$ is the SML depth, on the fresher side than on the saltier side of the front (Fig. 1-7; for other cases, see Fig. S7). This leads to a lateral temperature difference $\left(\Delta T=d T_{s}-d T_{f}\right)$, which increases the density ratio to $R=\frac{2 \pi \cdot Q \cdot\left(\frac{1}{z_{f}}-\frac{1}{z_{s}}\right)}{f \cdot c_{p} \cdot \rho_{o} \cdot(\beta \alpha) \cdot \Delta S}$ within an inertial period.

As the across-front temperature difference increases toward fully compensating the salinity front, the vertical temperature inversion also compensates the vertical salinity restratification. Any further heat loss will cause a deepening of the fresh SML, mixing with warmer saltier water below the restratification, reducing the sharpness of the compensated salinity front and vertically redistributing the previously surface-trapped heat loss. Thus, the 

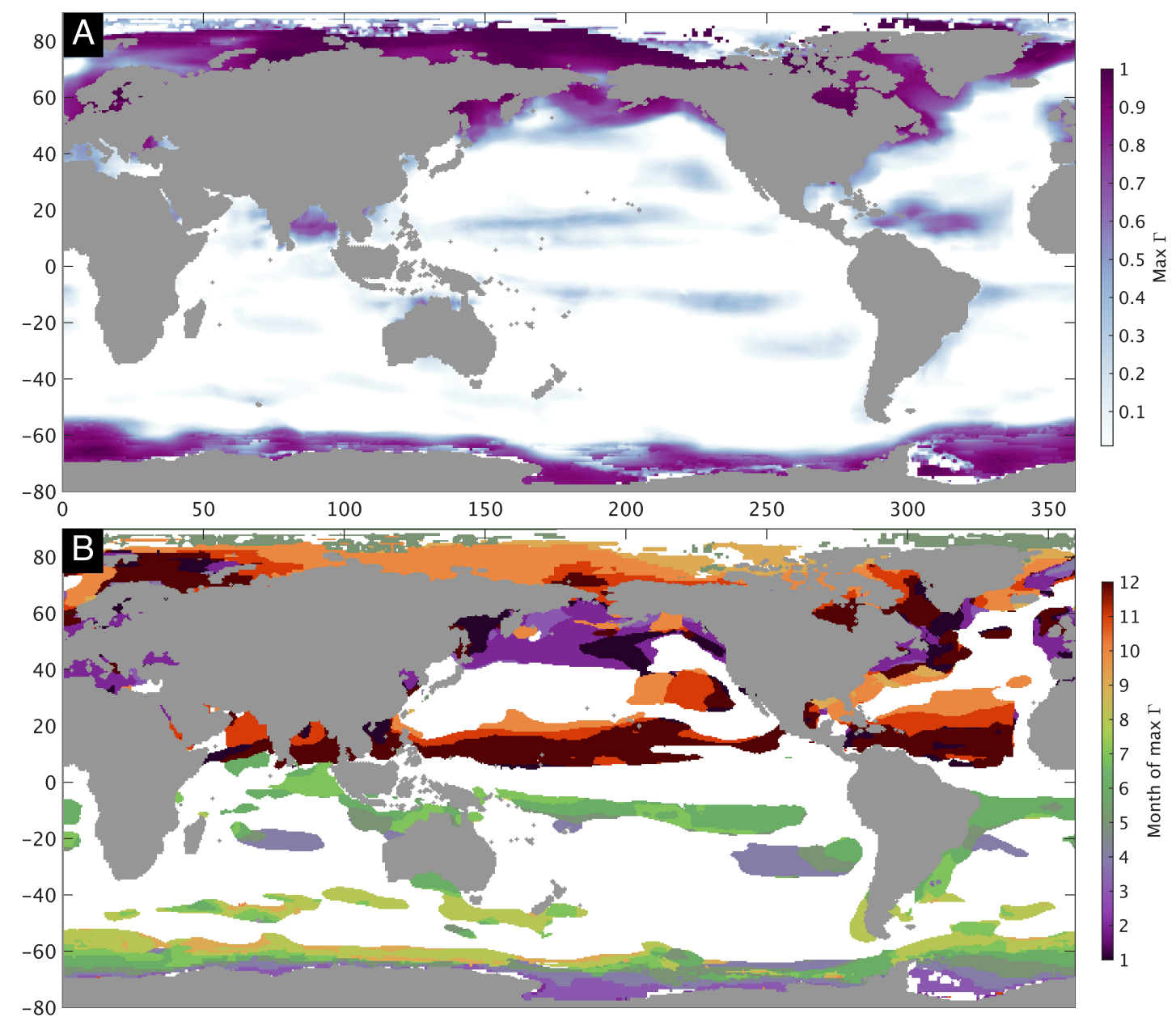

Figure 1-6: Climatological estimate of the global prevalence of cooling-induced T-S compensation. (A) Possible occurrence of cooling-induced compensation where both the SML is cooled and density gradients are salinity-dominated. The estimate, cast as a likelihood between 0 and 1 , is based on a co-occurrence of the necessary factors calculated from a climatology of surface heat flux and horizontal density ratios (normalized factors designated $q$ and $r$ ), with greater weight placed on larger (negative) fluxes and stronger salinity control (smaller density ratio). The maximum $(\Gamma=q \cdot r)$ of the 12 months for each pixel is plotted in (A), and darker shades indicate more favorable conditions. (A) Month of the maximum $\Gamma(1=$ January, $12=$ December $)$, generally corresponding to local winter months.

density ratio of these surface fronts tends toward 1 but does not exceed 1 .

In general, the time scale to attain full compensation is longer than one inertial period. For example, a 0.5 -psu salinity front at $20^{\circ}$ latitude, with $10-\mathrm{m}$ shallow restratification next to a 50-m-deep SML, will become $33 \%$ compensated within an inertial period forced by a cooling flux $\left(150 \mathrm{~W} / \mathrm{m}^{2}\right)$ and thus fully compensated within three inertial periods. 


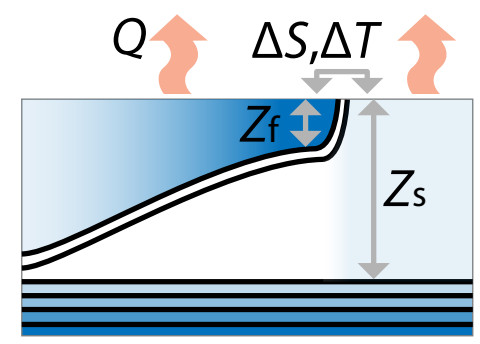

Figure 1-7: Idealized SSS submesoscale front, where the shallow restratification is due to the slumping of the lateral salinity gradient. Temperature is shaded in color, and contours of density are in black.

\subsection{Concluding Remarks}

Beyond demonstrating a source of $T-S$ coherence at small scales, the findings point to the importance of considering salinity fields when interpreting SST. Commonly, SST gradients are interpreted as density gradients, and cold filaments are associated with dense water that is exchanged with the subsurface. However, in salinity-controlled regions, temperature fronts might actually be oppositely oriented density fronts (the colder side being more buoyant) that are controlled by salinity, and cold filaments may represent enhanced surface stratification instead of deeper mixed layers. In nutrient-poor regions, we would not expect enhanced phytoplankton productivity at these cold filaments, whereas in nutrient-rich but light-limited regions, we would expect enhanced biological growth in these types of cold shallow filaments.

Of further interest is the potential contribution that active surface compensation might make to the subduction of fresher water, water mass formation, and enhancement of isopycnal $T$-S variance or spice that can be stirred along isopycnals. Submesoscale fronts are ubiquitous in the ocean and are important for the cascade of tracer variance and energy from large-scale forcing to scales where they are dissipated. Submesoscale fronts are also associated with significant localized vertical velocities, connecting the surface with subsurface waters. Our analysis of observations and numerical modeling show that in regions where salinity defines the density gradients, surface cooling leads to the selective weakening of submesoscale density fronts by $T-S$ compensation and forms cold SST filaments at the most stratified submesoscale frontal boundaries. 


\subsection{Chapter Acknowledgments}

Acknowledgments: We are grateful to the captain and crew of the $R / V$ Roger Revelle, cochief scientist of the cruise E. L. Shroyer, and all ASIRI-OMM (Air-Sea Interaction Regional Initiative-Ocean Mixing and Monsoons) scientists, including A. Tandon, D. Sengupta, J. T. Farrar, R. A. Weller, M. M. Omand, A. F. Waterhouse, V. V. S. S. Sarma, and A. L. Gordon. Funding: This work was carried out under the Office of Naval Research's ASIRI (grants N000141612470 and N000141310451) in collaboration with the Indian Ministry of Earth Science's OMM initiative supported by the Monsoon Mission. 


\subsection{Supplementary Material}

\section{Satellite data}

Sea surface salinity (SSS) from NASA's SMAP satellite (available since 2015, but not at the time of the cruise in 2013) gives a more detailed view (at 1/4 patial resolution) of SSS gradients in the Bay of Bengal compared to the Aquarius satellite data (shown for 2013 in Fig. 1-2). The SMAP satellite data from early December 2015 (Fig. 1-8) shows a correspondence between elevated chlorophyll and patches of cold water in the AS, but no such correspondence is seen in the BoB. In addition, Fig. 1-8 shows strong gradients in SSS in the northern BoB, which correspond to SST features. While the cold patch centered around 88 is also a minimum in SSS, the cold filament centered around 92 corresponds to an SSS front, a likely region of strong and shallow restratification. Further supporting this interpretation is the lack of any enhancement of chlorophyll in the filament around 92 , even though it is as cold as the patches in the AS that show elevated chlorophyll.

\section{Compensation between salinity and temperature for fronts of varying strength}

To calculate the gradient-scale-specific distribution of density ratios (Fig. 1-4), the thermosalinograph timeseries of $S$ and $T$ are spatially gridded, with each of the 6 straight sections uniformly interpolated at $0.3 \mathrm{~km}$ resolution. These sections are then passed through a multilevel one-dimensional wavelet (Daubechies-1) decomposition. At each level (scale) of this decomposition, we difference the adjacent coefficients. These co-located $\alpha \Delta S$ and $\beta \Delta T$ co-

efficients are plotted versus each other in Fig.1-9 for a particular level (corresponding to a wavelet scale of $0.6 \mathrm{~km}$ ). The ratio of $\alpha \Delta S$ and $\beta \Delta T$ coefficients is equal to the local density ratio at the respective scale of the decomposition level. The distribution of points shows a compensating tendency, with a median density ratio $R=0.4$. Small values of $\alpha \Delta S$ and $\beta \Delta T$ clustered around the origin are associated with very weak fronts and are of much less significance than large values of $\alpha \Delta S$ and $\beta \Delta T$. Hence, the 95th percentile values are indicated by purple bars in Fig. 1-4. 

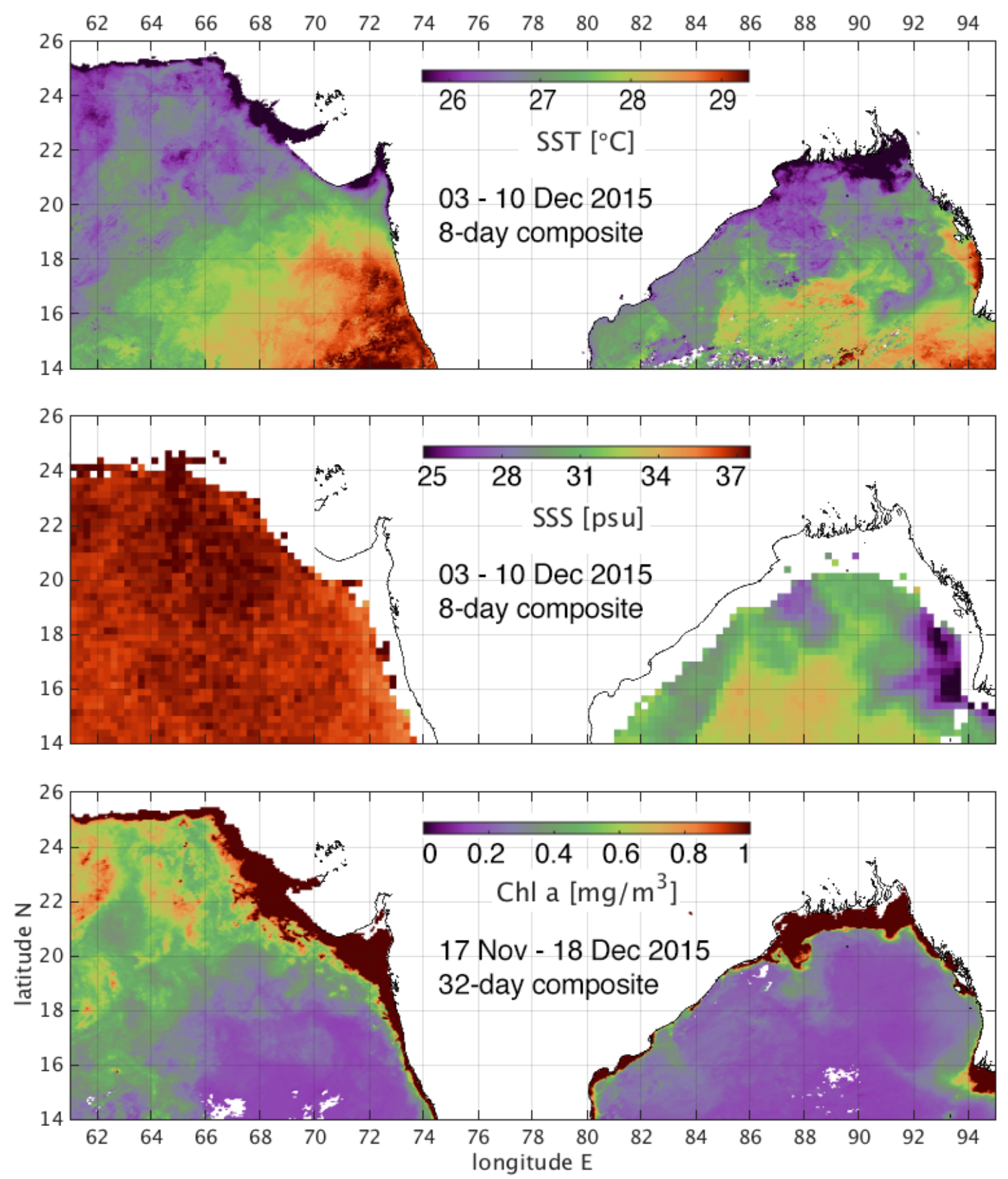

Figure 1-8: Similar to Fig.1-2, but for winter 2015. (a) Night-time temperature (SST); (b) salinity (SSS); both are 8-day composites. (c) Chlorophyll-a, 32-day composite. Note the absence of chlorophyll in the cold filaments of the BoB, compared to the enhanced productivity in the cold patches of the Arabian Sea. Data source/satellite: NASA SMAP and MODIS/Aqua. 


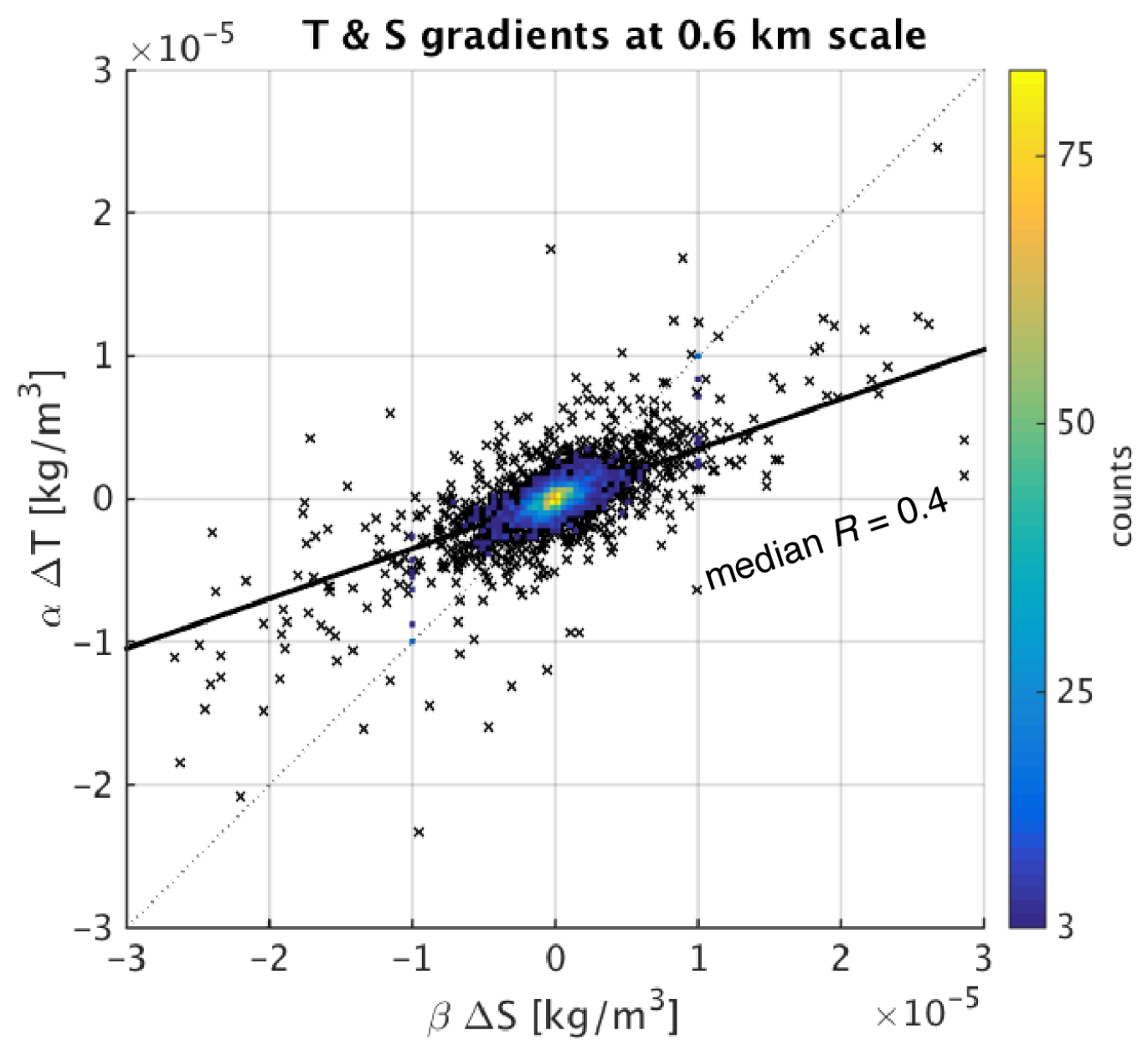

Figure 1-9: Scatterplots of density variability at wavelength of $0.6 \mathrm{~km}$, due to salinity and temperature gradients along the longest straight section from the cruise (Leg-2 section-A). The points clustered near the origin correspond to very weak density gradients, their countdensity is colored over the point cluster. The black line, which fits the data, has a slope $=0.4$, while the dotted line of slope $=1$ indicates perfect density compensation.

\section{Density compensation at a salinity front illustrated in a one-dimensional frame- work}

To illustrate the mechanism by which cooling leads to density compensation at a salinity front, a series of $T$-S profiles forming a front are individually cooled in a one-dimensional model framework (Fig. 1-10). Initially, the profiles have a uniform temperature, a background salinity stratification that generates a vertical buoyancy gradient $N^{2}=10^{-5} \mathrm{~s}^{-2}$, and a halocline with peak $N^{2}=10^{-2.8} \mathrm{~s}^{-2}$ in accordance with the upper ocean stratification observed in UCTD profiles from the BoB (Fig.1-3). The horizontal spacing of profiles in this model is arbitrary and therefore horizontal differences are not strictly gradients. 
But, non-dimensional quantities such as relative changes and density ratios are valid and instructive.

A constant surface cooling of $-50 \mathrm{~W} \mathrm{~m}^{-2}$ is applied for 1 week in time increments of 60 minutes, allowing the profiles to convectively adjust at each time step. The evolution of the profiles in response to cooling (Fig. 1-10) shows how the typical stratification beneath a front in a salinity-controlled region leads to density compensation of the sharp and shallow mixed-layer front when subject to atmospheric cooling.

Surface $T$ - $S$ clearly shows the largest temperature drop to be coincident with the front (Fig. 1-10e). The density ratio $R$ shifts towards positive values at the front, approaching 1 . Away from the front, the salinity gradient is reinforced by a temperature gradient, leading to negative $R$, albeit for weak density gradients. The peak magnitude of the density front is reduced by about $50 \%$, as the temperature difference between the deeper saltier side and the shallower fresh side compensates the salinity difference.

\section{Model sensitivity tests}

The results of the modeling study are found to be robust, over a range of parameter and configuration choices for the horizontal diffusivity, horizontal resolution and surface cooling flux. In addition to the simulation presented in the article, model simulations are conducted with a 5 -fold and 25-fold increase in horizontal diffusivity, half the horizontal grid resolution, without a cooling flux and with a continuous cooling flux. The significant results are not sensitive to these parameter changes, the enhanced $T-S$ compensation at $\mathrm{O}(1) \mathrm{km}$ scales is a robust result of the restratifying salinity front being cooled for a period to sufficiently change the SST so that it partially compensates the sharp salinity gradients.

The following is an overview of the results from model simulations conducted with varying model parameters.

We chose the diffusivity range of 0.2 to $5 \mathrm{~m}^{2} / \mathrm{s}$ in accordance with the findings of the LatMix observational campaign (Shcherbina et al., 2015), in which the "background" value of horizontal diffusivity based on the lateral dispersion of tracers at $\mathrm{O}(1 \mathrm{~km})$ scales was found to be about at $1 \mathrm{~m}^{2} / \mathrm{s}$.

The horizontal diffusivity affects the scale of the instabilities that emerge from the initially uniform front in the model. As expected, a lower value of diffusivity permits smaller scale features, while a higher value reduces small-scale variance. However, for the plausible 
(a)
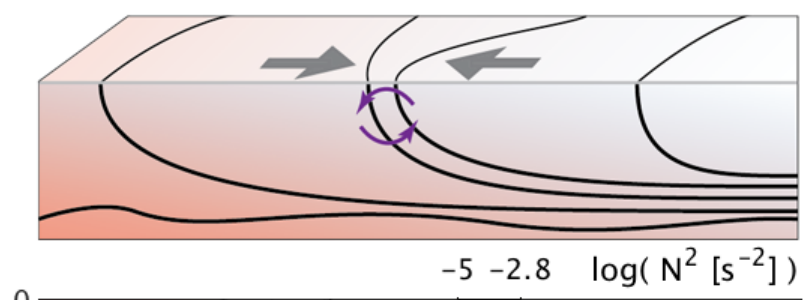

(b)

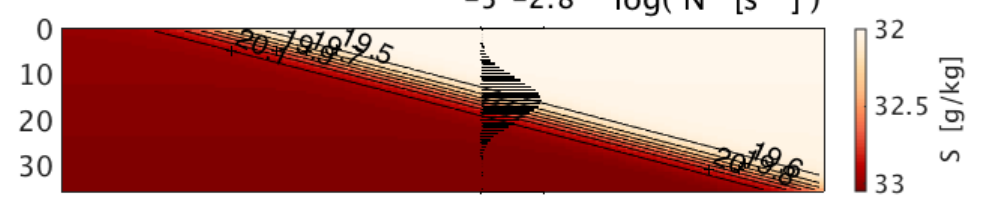

(c)

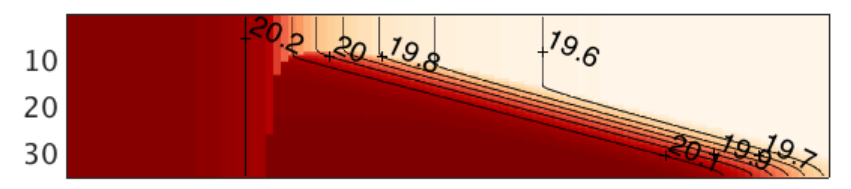

(d)

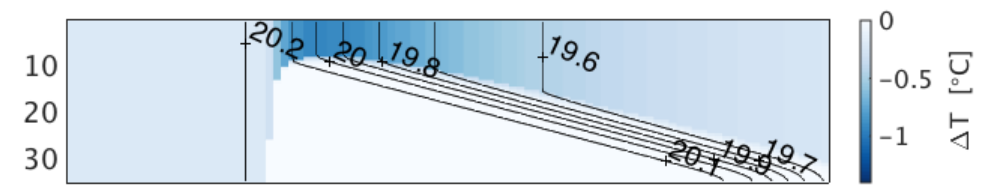

(e)

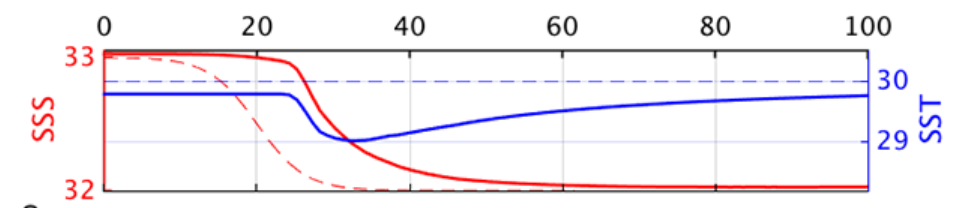

(f)

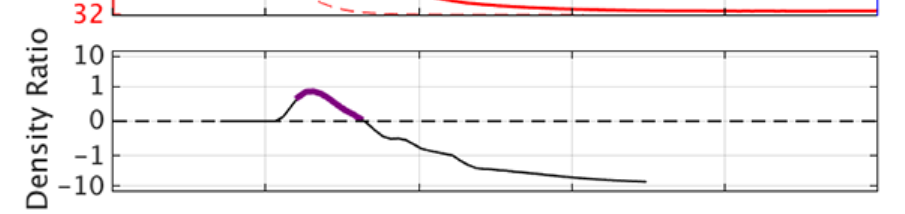

(g)

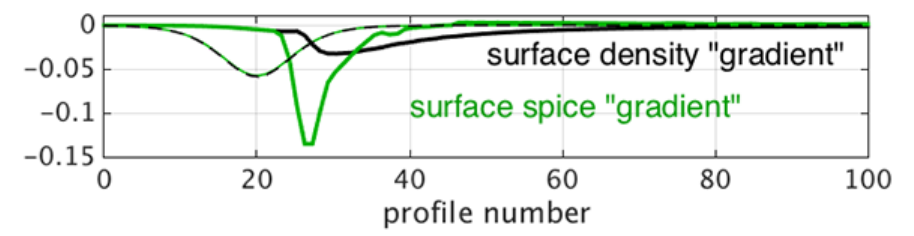

Figure 1-10: Illustration of active compensation and creation of spice variance with a series of one-dimensional (1-D) column models. A. Conceptual setting: in a region where salinity controls large-scale density gradients, mesoscale currents stir and strain fronts. Submesoscale circulations slump fronts and create shallow salinity stratification. B. Idealized initial salinity front and stratification modeled by a series of 1-D salinity profiles, with temperature set initially uniform. B. Salinity after 1 week of constant cooling at the rate of -50 $\mathrm{W} / \mathrm{m}^{2}$ applied to the top of profiles. D. Temperature anomaly after 1 week. E. SSS and SST before and after cooling (dashed and bold). SST is coldest at the front; its sharpest gradient is coincident with the sharp SSS gradient. F. Density Ratio $R$ approaches 1 at the sharp front (purple), while it increases to large negative values in regions of small tracer gradients (black). G. Horizontal gradients of density (black) and spice (green). Cooling leads to a circa $50 \%$ reduction in the magnitude of the density gradient, and circa $100 \%$ increase in spice gradient. 


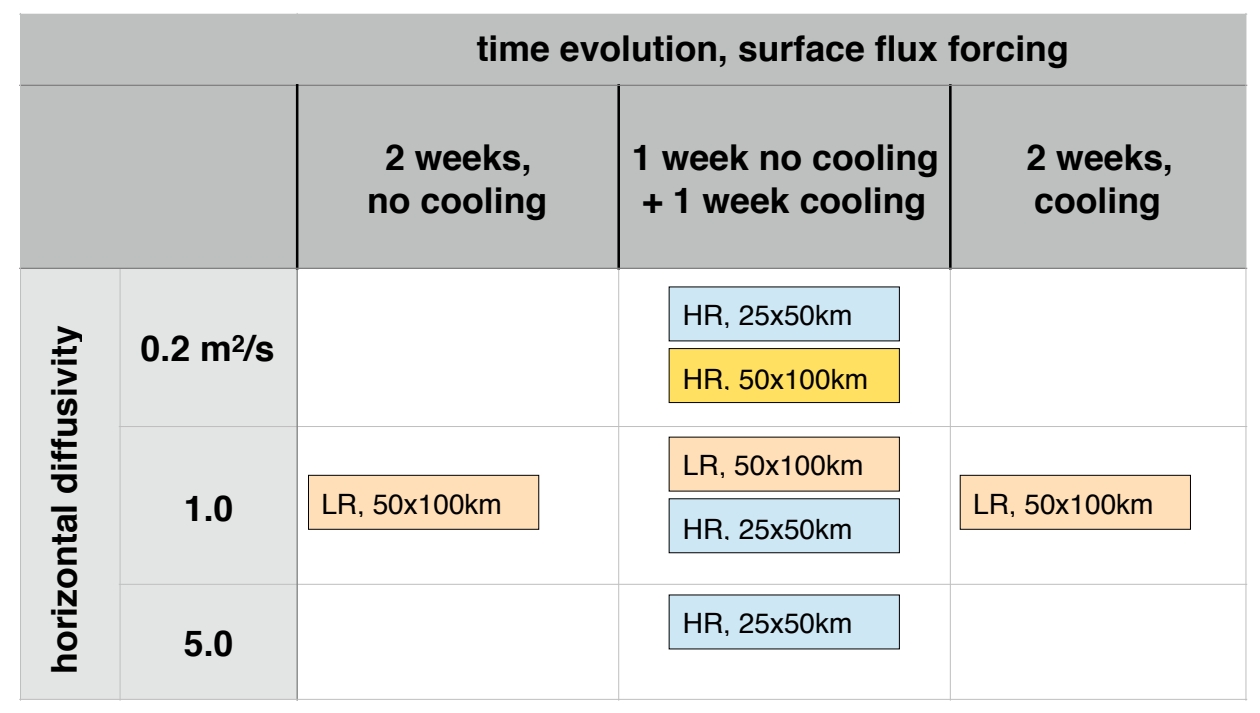

Figure 1-11: Paramter-configuration space of sensitivity tests. $\mathrm{HR}=$ higher horizontal resolution $(0.25 \mathrm{~km}), \mathrm{LR}=$ lower horizontal resolution $(0.5 \mathrm{~km})$. Yellow box marks the simulation presented in the main article.

range of horizontal diffusivities tested, the model always produces instabilities that restratify at submesoscales, and thus the compensation mechanism by cooling is active in all cases. For the $0.25 \mathrm{~km}$ resolution simulation presented in the main article, we used a horizontal diffusivity of $0.2 \mathrm{~m}^{2} / \mathrm{s}$, which produced surface salinity variability on scales similar to the observed variability in the TSG transects.

The process of restratification occurs at submeso-scales of order (1) km, and our model resolution of $0.25 \mathrm{~km}$ resolves the eddies that generate restratification. Additional simulations with a coarser horizontal resolution of $0.5 \mathrm{~km}$ contains similar eddies that restratify the SML, albeit the fronts that develop are slightly weaker at the coarser resolution. Both simulations develop submesoscale compensation and even the $0.5 \mathrm{~km}$ resolution model demonstrates the mechanism put forth in article.

We consider the 1st week of simulation as spin-up for the model, since we start from an idealized initial condition based on a horizontal interpolation between 2 measured vertical profiles of temperature and salinity. It takes about a week for a more realistic flow field to develop from the initially uniform front.

Model simulations are also conducted (a) without cooling (to test our null hypothesis), and (b) with surface cooling applied throughout the model run, i.e. even during the spin-up phase of the model. Without cooling, the fronts develop instability, slump, and restratify 


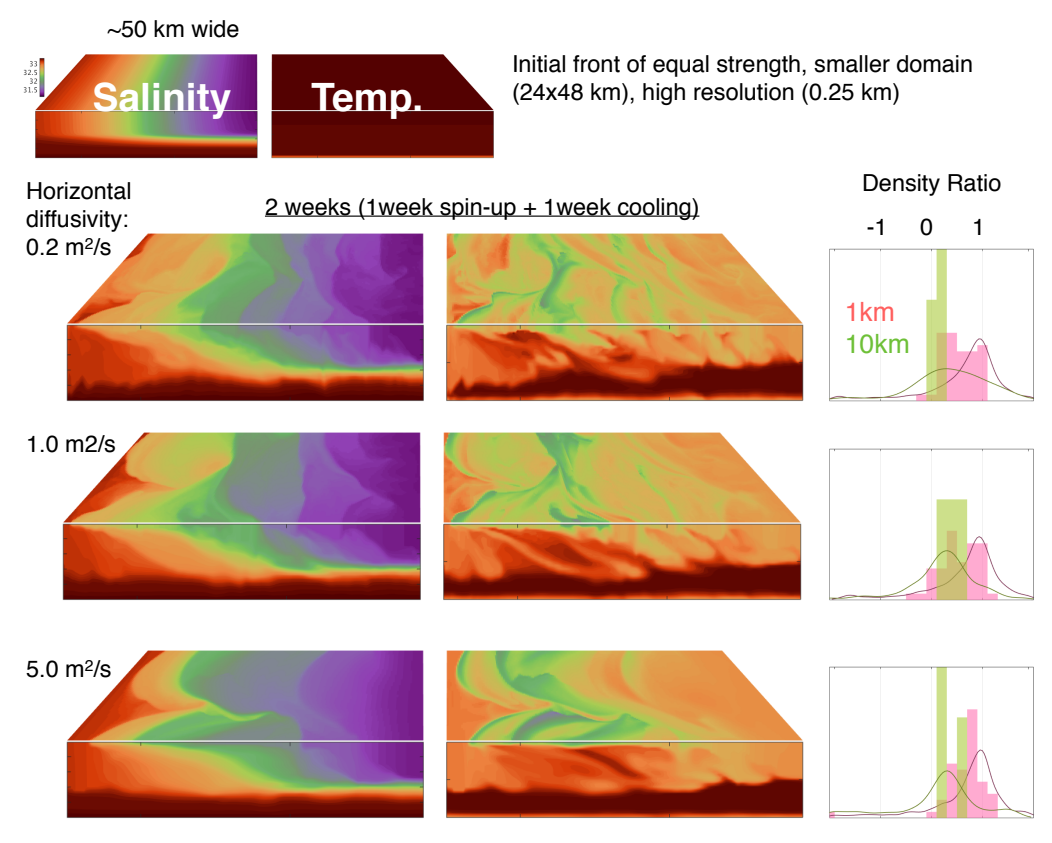

Figure 1-12: Simulations assessing the model's sensitivity to the value of horizontal diffusivity, set at $0.2,1.0$ and $5.0 \mathrm{~m}^{2} / \mathrm{s}$. The three model runs are started from the same initial conditions and forced with the same heat flux. While the growth of the instabilities varies between the simulations (lower diffusivity simulations exhibit more fine-scale features than the higher diffusivity simulation), the pertinent result of enhanced $T$-S compensation at $\mathrm{O}(1) \mathrm{km}$ scales compared to $\mathrm{O}(10) \mathrm{km}$ scales remains unaffected.

the SML, but no density compensation occurs. When the surface is cooled from the start of the model run, the $T$-S compensation increases at all scales since the fresh side is already shallower than the salty side by the end of the first week. Thus, by the time the front goes unstable and before eddies develop in the model, the "base state" is already weakly compensated. In effect, this is equivalent to initializing the front with two $T$-S profiles that are partially compensated, instead of a horizontally uniform temperature. The submesoscale compensation at the restratifying fronts occurs no matter whether the initial front is weakly compensated or not, and is stronger than the compensation of the "base state" prior to the fronts becoming unstable.

\section{Vertical density compensation: Weakening the density stratification by cooling}

Besides frontal compensation, surface cooling also leads to vertical compensation of the salinity stratification by temperature inversion. The vertical salinity gradient beneath the SML on the shallower side of the front limits the amount of cooling possible before convective 

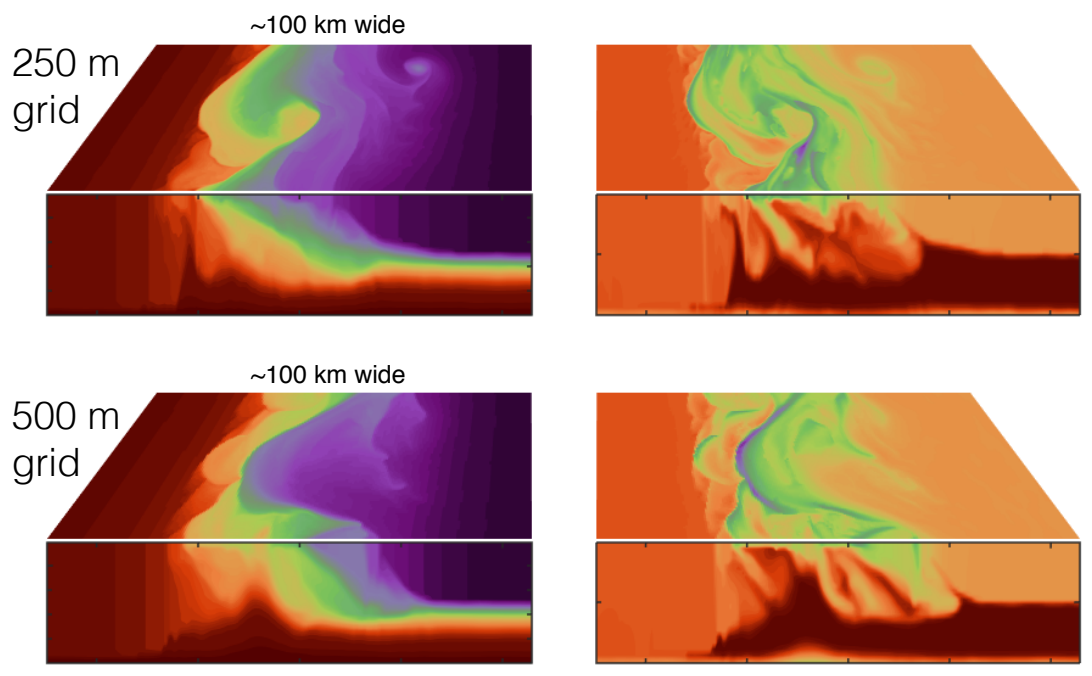

Figure 1-13: Tests assessing the sensitivity of the simulation to the horizontal grid resolution, set at $0.25 \mathrm{~km}$ and $0.5 \mathrm{~km}$, for the same domain $(48 \times 96 \mathrm{~km})$ and the same value or horizontal diffusivity $\left(1.0 \mathrm{~m}^{2} / \mathrm{s}\right)$. The two model runs were started from the same initial condition and forced with the same heat flux. The pertinent result of enhanced $T-S$ compensation at $\mathrm{O}(1) \mathrm{km}$ scales compared to $\mathrm{O}(10) \mathrm{km}$ scales remains unaffected.

mixing deepens the layer, distributing both the heat loss and the fresher water in the SML downwards, deepening the SML and weakening the front. Thus, cooling would initially drive a front that is based purely on salinity towards density-compensation by creating an opposing temperature gradient. Continued cooling would deepen and weaken the initial salinity gradient and decrease the temperature gradient across front, and move $R$ back towards zero (Fig. 1-15, top row).

For the simplest salinity-front geometry where the SML-depth is shallower on the fresh side, and the vertical and horizontal salinity differences are equal, the maximum possible density ratio $R$ increases as the ratio of fresh-to-salty SML depth decreases, and tends towards $R=11$.1. For alternate geometries of salinity fronts and stratification in which the fresher side is not necessarily shallower, nor the horizontal and vertical salinity gradients equal, $R$ can tend towards values other than 1 (Fig. 1-15). 


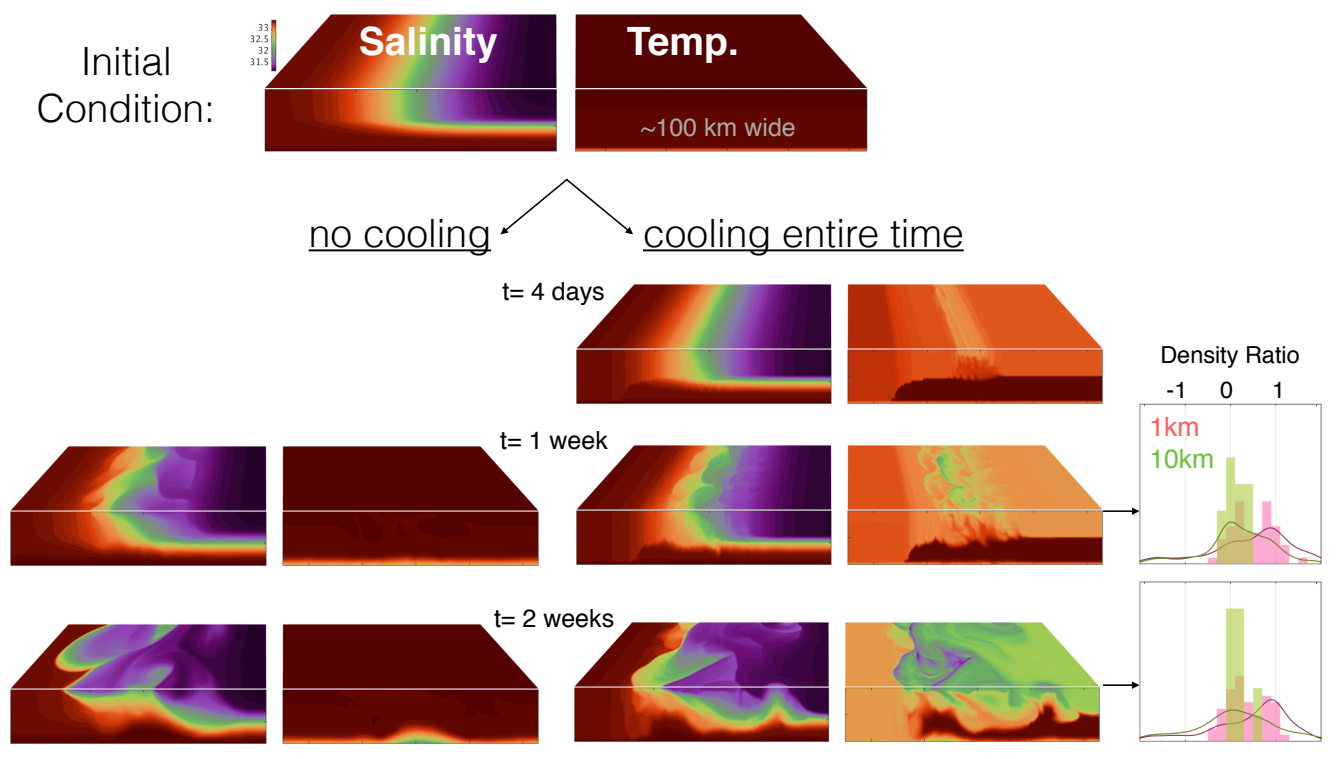

Figure 1-14: Model tests run with the same initial conditions under two different cooling scenarios: (a) no cooling for 2 weeks, and (b) constant cooling of $-50 \mathrm{~W} / \mathrm{m}^{2}$ for 2 weeks (instead of 1 week of no cooling and 1 week of cooling in all other simulations). The unforced simulation (left) develops similar frontal instabilities, however lacks the fine-scale $T-S$ variance and compensation (right). The simulation that is cooled during the 1 st week, when the frontal instabilities are still growing, develops the same characteristic compensation enhanced at the $\mathrm{O}(1) \mathrm{km}$ scale as the simulations presented in the article.

$$
\begin{array}{r}
d T=\frac{Q}{c_{p} \rho Z} \\
\Delta_{h} T=\frac{Q}{c_{p} \rho}\left(\frac{1}{Z_{\text {fresh }}}-\frac{1}{Z_{\text {salty }}}\right) \\
R=\frac{\alpha \Delta T}{\beta \Delta S} \\
R_{v}=\frac{\alpha \Delta_{v} T}{\beta \Delta_{v} S}=\frac{\alpha}{\beta} \frac{Q}{c_{p} \rho} \frac{1}{Z} \frac{1}{\Delta_{v} S} \\
R_{h}=\frac{\alpha}{\beta} \frac{Q}{c_{p} \rho} \frac{\left(\frac{1}{Z_{\text {fresh }}}-\frac{1}{Z_{\text {salty }}}\right)}{\Delta_{h} S}
\end{array}
$$

to remain stably stratified: $R_{v} \leq 1, \frac{\alpha}{\beta} \frac{Q}{c_{p} \rho} \leq Z_{f} \Delta_{v} S$

$$
\max R_{h}=\frac{\Delta_{v} S}{\Delta_{h} S}\left(1-\frac{Z_{\text {fresh }}}{Z_{\text {salty }}}\right) \quad\left[\text { for } Z_{\text {fresh }}<Z_{\text {salty }}\right]
$$



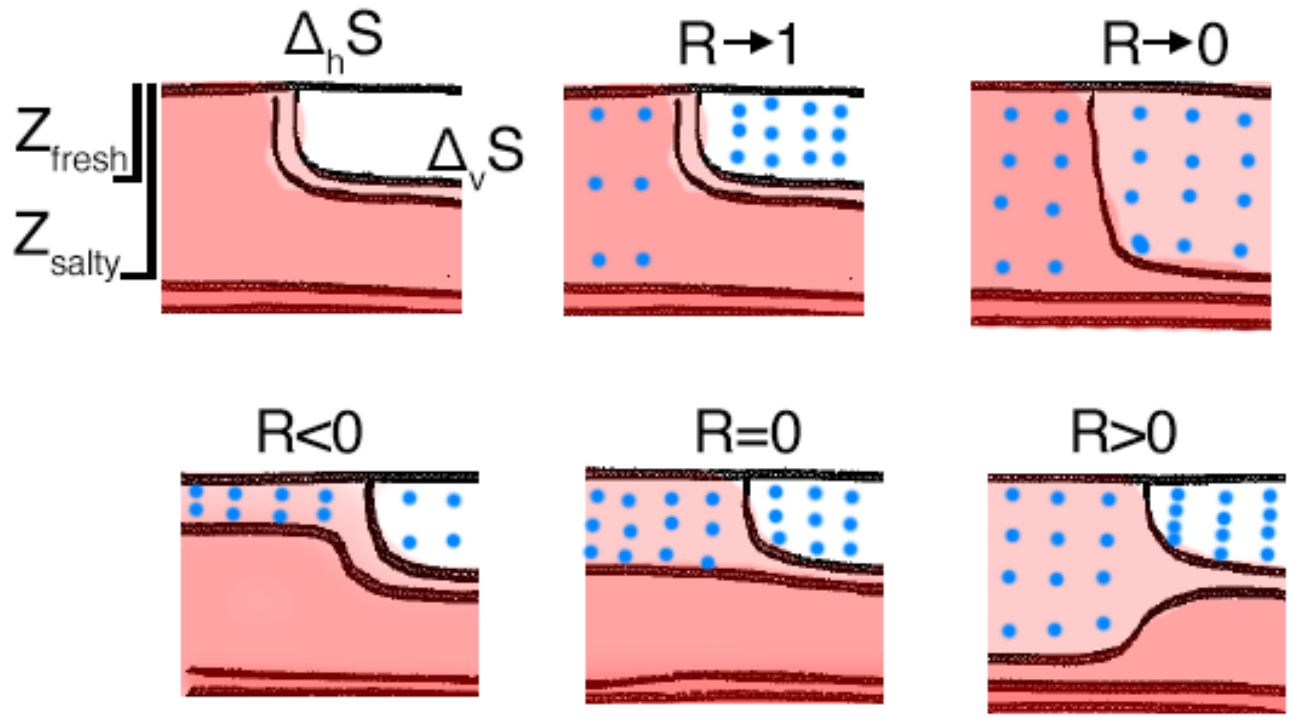

Figure 1-15: Schematic of simple salinity fronts and stratification - red shades indicate salinity, blue dots represent heat loss. Top, left to right: Front that tends towards densitycompensation when cooled; until the vertical T-inversion matches the S-stratification, at which point the front is deepened and weakened. Lower Row. Front and stratification geometries, which when cooled lead to $T$-gradients either reinforcing (left), not affecting (middle), or compensating (right) the salinity-density front. 
THIS PAGE INTENTIONALLY LEFT BLANK 


\section{Chapter 2}

\section{How Spice is Stirred in the Bay of Bengal}

\section{$2.1 \quad$ Abstract}

The scale-dependent variance of tracer properties in the ocean bears the imprint of the oceanic eddy field. Anomalies in spice (which combines anomalies in temperature $(T)$ and salinity $(S)$ on isopycnal surfaces) act as passive tracers beneath the surface mixed layer (ML). We present an analysis of spice distributions along isopycnals in the upper $200 \mathrm{~m}$ of the ocean, calculated with over 9000 vertical profiles of $T$ and $S$ measured along $\sim 4800 \mathrm{~km}$ of ship tracks in the Bay of Bengal. The data are from three separate research cruises in the winter monsoon season of 2013, and in the late and early summer monsoon seasons of 2015 and 2018. We present a spectral analysis of horizontal tracer variance statistics on scales ranging from the submesoscale $(\sim 1 \mathrm{~km})$ to the mesoscale $(\sim 100 \mathrm{~km})$. Isopycnal layers that are closer to the ML-base exhibit redder spectra of tracer variance at scales $\lesssim 10 \mathrm{~km}$ than is predicted by theories of quasi-geostrophic turbulence or frontogenesis. Two plausible explanations are postulated. The first is that stirring by submesoscale motions and shear dispersion by near-inertial waves enhance effective horizontal mixing and deplete tracer variance at horizontal scales $\lesssim 10 \mathrm{~km}$ in this region. The second is that the spice anomalies are coherent with dynamical properties such as potential vorticity, and not interpretable as passively stirred. 


\subsection{Introduction}

The stirring and mixing of properties by the oceanic flow field is of longstanding interest for understanding the dispersal and distribution of substances such as heat, salt, dissolved gases, nutrients, phytoplankton, and pollutants. Conversely, the patterns of chemical and physical tracer distributions in the ocean can be used to interpret the dynamical characteristics of the flow. Within the vast range of spatial scales on which ocean currents and eddying motions act to stir properties, the submesoscale, and related dynamics, remain difficult to observe.

Submesoscale processes manifest at spatial scales $O(1-10) \mathrm{km}$, exhibit instabilities on timescales that compare with near-inertial waves, and play a key role in the vertical transport of properties, such as nutrients and buoyancy (Lévy et al., 2012; Thomas et al., 2008b). They also facilitate the downscale cascade of energy through the loss of balance of mesoscale flows (Capet et al., 2008b), thereby connecting the larger $O(100) \mathrm{km}$ mesoscale with the smaller $(<10 \mathrm{~m})$ dissipative scales. Observations of thermohaline compensation at submesoscales in the surface mixed layer, dye releases, and drifter dispersion at fronts, have shown the key role of frontal dynamics in controlling horizontal mixing (Rudnick and Martin, 2002b; Shcherbina et al., 2015; D'Asaro et al., 2018). But below the surface mixed layer, classically the interior domain of isopycnal stirring, the role of submesoscale dynamics and forward energy cascades remains unclear (McWilliams, 2016), and the effect of a downscale kinetic energy transfer in the submesoscale regime on tracer variance (Badin et al., 2011) has not been observed.

Measurements of submesoscale dynamics have been stymied by the difficulty of synoptically observing a three-dimensional flow field in an area that is tens of kilometers wide, while resolving features that are a kilometer or sub-kilometer in extent and rapidly evolving in time. Additionally, direct measurement of the kinetic and potential energy of such flows is dominated by oscillatory motion and internal waves that mask the signal of vortical (nonwave) submesoscale dynamics (Callies and Ferrari, 2013). While recent studies have made progress in observing submesoscale signatures (Thompson et al., 2016), challenges remain

in directly measuring the submesoscale flow fields that stir tracers. Oceanographers have instead examined the variability of tracer fields on isopycnal surfaces to infer the underlying dynamics (Kunze et al., 2015; Klymak et al., 2015).

We present a new look at along-isopycnal tracer variability on scales ranging from 1- 
$100 \mathrm{~km}$, from a synthesis of data collected during three field studies in the Bay of Bengal (BoB). This large basin in the northern Indian Ocean has unique characteristics: (i) It is strongly stratified in the upper $50 \mathrm{~m}$ by freshwater inputs, (ii) it contains distinct water masses, and hence, large-scale gradients of spice, a passive tracer, (iii) it hosts energetic eddies, and (iv) it is forced by both consistent winds and impulsive storms. It is thus a prime location for studying the dynamics of the upper ocean, both within the highly stratified pycnocline layers at the base of the mixed layer, and within the weaker pycnocline beneath.

Our analysis of these new observations of water mass distributions in the BoB reveals statistics inconsistent with existing descriptions of tracer stirring in the ocean. The observed tracer gradient distributions within the shallower stratified pycnocline do not support quasigeostrophic or ageostrophic theories of homogeneous turbulence. The relative paucity of tracer variance below $10 \mathrm{~km}$ scales compared to larger scales in our observations cannot be easily explained. We speculate that, most likely, submesoscale motions (vortical or wave) play a more important role than was previously envisaged in stirring tracer and dissipating its variance in the ocean's near-surface interior.

In what follows, we begin by reviewing theories of ocean stirring (Section 2). We then describe our new observations, with some details about the instruments and measurements in Section 3. Our methods for parsing through the ship sections and constructing spectra of along-isopycnal tracer variance are described in Section 4, and our statistical results are presented in Section 5. In order to provide context to our findings we compare the spectra calculated in the BoB to those from the Pacific and Atlantic Oceans (Section 6), and provide potential explanations for the differences. We provide brief conclusions in Section 7. Supplementary figures are included.

\subsection{Oceanic Variance Spectra}

Tracer gradients exist across a wide range of spatial scales in the ocean, from thousands of kilometers down to millimeters, and are connected by a down-scale variance cascade (Smith and Ferrari, 2009). Gradients are stirred, strained, and sharpened by motions ranging from the ocean gyres, eddies, and internal waves, to small enough scales where three-dimensional turbulence and molecular diffusion ultimately mix properties (Shcherbina et al., 2015). The 
large-scale currents, with scales $O(10) \mathrm{km}$ or more, are described by balanced sub-inertial quasi-geostrophic (QG) dynamics, which exhibits an upscale transfer of energy. At the smallest scales of $O(10) \mathrm{m}$ in the ocean interior, shear instabilities and breaking internal waves produce isotropic turbulence that effectively mixes tracers and erases tracer gradients and variance (MacKinnon et al., 2013). In between these scales, in the regime of submesoscale dynamics, both wave dynamics, frontal instabilities, and vorticity containing modes are relevant (Polzin and Ferrari, 2004; McWilliams, 2016).

The typical scales of the motions stirring the ocean determine the distribution of the typical scales of tracer variance. The statistical distribution of tracer variance across these scales can be quantified by a spectral power decomposition $\left(\mathcal{P}_{k}\right)$ as a function of horizontal wavenumber $k$, providing a check on theories that attempt to describe the fluid dynamics, and test the relative importance of $\mathrm{QG}$ and submesoscale dynamics in stirring the ocean. Unlike theoretical studies, observational studies often plot tracer variance spectra as gradient spectra $\mathcal{G}_{k}$ (multiplying $\mathcal{P}_{k}$ by $4 \pi^{2} k^{2}$ ), either because gradients are the directly measured quantity, or to be able to visually distinguish between variance spectral slopes more easily. We follow this convention, but in the text, we refer to variance spectra as power laws $\mathcal{P}_{k} \sim k^{m}$, and append the whitened gradient spectra power law $\left(\mathcal{G}_{k} \sim k^{m+2}\right)$ in parenthesis to avoid misinterpretation.

Statistics of tracer patterns relate to statistics of the motions that create those patterns. The following descriptions (schematically shown in Fig.2-1) are set in the framework of 2-dimensional, homogeneous, stationary macro-turbulence. Making the simplifying assumption of the existence of a turbulent inertial range between the forcing scale (e.g. the baroclinic Rossby radius of deformation) and the turbulent dissipation scale, the power spectrum for kinetic energy $E_{k}$ obeys several different power laws. The tracer's variance spectrum $\mathcal{P}_{k}$ is related to the kinetic energy spectrum $E_{k}$. If $E_{k} \sim k^{-n}$, then in geostrophic turbulence theory, $\mathcal{P}_{k} \sim k^{(n-5) / 2}$ for $n<3$ (Callies and Ferrari, 2013). QG theory for the ocean's interior predicts a kinetic energy spectrum following a power law with $n=3$, thus a tracer spectrum $\mathcal{P}_{k} \sim k^{-1}\left(\mathcal{G}_{k} \sim k^{+1}\right)$. Similarly, if $n>3$, i.e. the kinetic energy spectrum falls off more rapidly, large-scale motions dominate, and the non-local stirring leads to a Batchelor spectrum, $\mathcal{P}_{k} \sim k^{-1}$ (Batchelor, 1959), equivalent to the interior QG tracer spectrum.

An extension of interior QG dynamics to the surface, called SQG theory, predicts steeper tracer spectra of $k^{-5 / 3}\left(k^{+1 / 3}\right)$ near the surface. But, the interior-QG spectrum still holds at 
greater depths, as small-scale motions are attenuated with depth (Lapeyre and Klein, 2006). Non-QG theories predict more energy at smaller scales, leading to more tracer stirring at smaller scales. Stratified turbulence theory predicts that turbulent patches with longand-thin aspect ratios (for example, "blinis" or "pancakes" formed from the geostrophic adjustment of water patches mixed by breaking near-inertial waves (Sundermeyer et al., 2005)) can steepen the tracer variance spectrum in the ocean's interior to follow an ObhukovCorrsin spectrum with $k^{-5 / 3}\left(k^{+1 / 3}\right)$ (Brethouwer and Lindborg, 2008). Frontogenesis and ageostrophic dynamics sharpen gradients into fronts more quickly; a series of sharp fronts

yields a spectrum with a $k^{-2}$ decay of tracer variance, and a flat $\left(k^{0}\right)$ gradient spectrum (Klein et al., 1998).

Even though precise predictions of spectral slopes emerge from the different dynamical theories, their robustness and relevance to actual ocean states is not certain. Theoretical tracer variance distributions are based on assumptions of an inertial range and statistical homogeneity of the flow field, yet it is not clear whether such idealised cascades exist, or whether they are disturbed by flow inhomogeneities such as coherent structures or persistent anisotropic currents, and energy inputs by instabilities across the submesoscale, topographic influence, bottom friction, or atmospheric forcing. Nonetheless, existing observational spectral studies (reviewed in the discussion) make frequent comparisons with the idealised theories, and observational spectra offer insights independent of testing predictions.

\subsection{Observations in the Bay of Bengal}

Our study uses hydrographic measurements to calculate statistics of tracer variance, analyzing the distribution of water mass anomalies. As part of two research programs supported by the Office of Naval Research, the Air Sea Interaction Research Initiative (ASIRI) and the ongoing Monsoon Intra-Seasonal Oscillations in the Bay of Bengal (MISO-BOB), the $R / V$ Roger Revelle and the $R / V$ Thomas $G$. Thompson completed multiple hydrographic surveys of the Bay of Bengal between 2013 and 2018. Vertical profiles of $T$ and $S$ were measured to depths of at least $200 \mathrm{~m}$ during the ship surveys. Two of the surveys made transects $(>100 \mathrm{~km})$ with closely spaced $(\leq 300 \mathrm{~m})$ vertical profiles, while one survey completed longer transects $(>500 \mathrm{~km})$ that were sampled more coarsely $(\leq 5 \mathrm{~km}$ spacing between vertical profiles), yielding a total of over 9000 profiles of $T$ and $S$. 


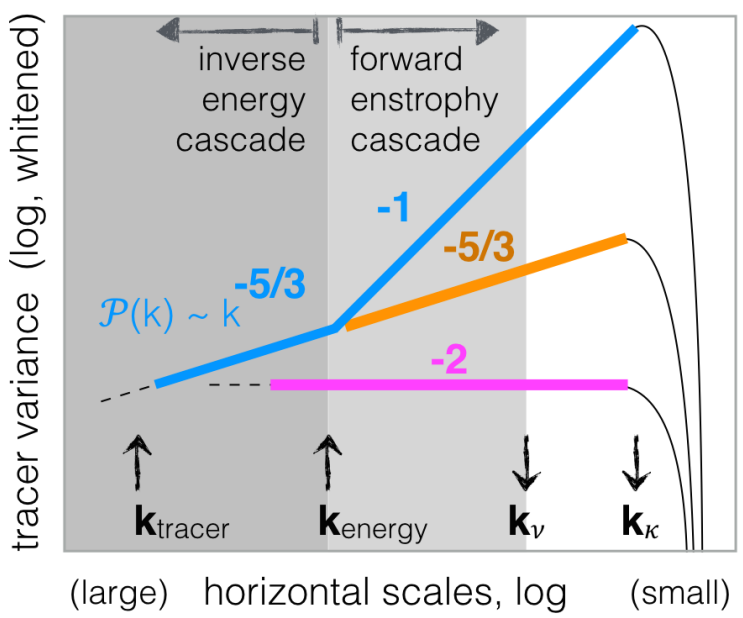

Figure 2-1: Schematic of passive tracer spectral distributions $\mathcal{P}(k)$, whitened (multiplied by $4 \pi^{2} k^{2}$ ) for clarity of presentation, and shown on log-log axes. Predictions of spectral shapes of a tracer stirred by 2-dimensional homogeneous turbulence, according to several theories: interior QG (blue), surface QG (orange), stratified "pancake" turbulence (orange), and frontogenesis (magenta). These predictions assume tracer variance in the ocean is introduced at the large scale (wavenumber $k_{\text {tracer }}$ ), cascades to smaller scales by the 2dimensional macro-turbulence, and is destroyed by 3-dimensional isotropic turbulent mixing. Energy is assumed to be injected at an intermediate scale $\left(k_{\text {energy }}\right)$ and cascades to larger scales in QG theory, producing a break in the predicted slope between the inverse energy cascade regime (where the kinetic energy spectrum $\mathcal{E}(k) \sim k^{-5 / 3}$, not shown), and the forward enstrophy cascade regime (where $\mathcal{E}(k) \sim k^{-3}$ ). While energy falls off rapidly in the viscous inertial regime (wavenumber larger than $k_{\nu}$ ), passive tracer variance does not fall off until the diffusive regime (wavenumber larger than $k_{\kappa}$ ).

\subsubsection{Environment}

The Bay of Bengal's unique hydrographic conditions are linked to the monsoonal circulation over the northern Indian Ocean, characterized by seasonally reversing winds and intense rainfall during the summer season. Both rainfall and river runoff contribute freshwater in excess of annual evaporation from the bay, lowering the surface salinity year-round (Fig.22A), with the lowest values observed a couple of months after the wet season, when river runoff reaches a maximum (Sengupta et al., 2006). The increased buoyancy of the surface mixed layer inhibits convective mixing (Jaeger and Mahadevan, 2018) during the colder winter months, and stratification limits the depth of wind-forced mixing throughout the year, isolating the sub-surface layers of the Bay of Bengal from direct ventilation (Jinadasa et al., 2016).

Evidence of this persistent insulation is seen in the pronounced oxygen minimum zone 

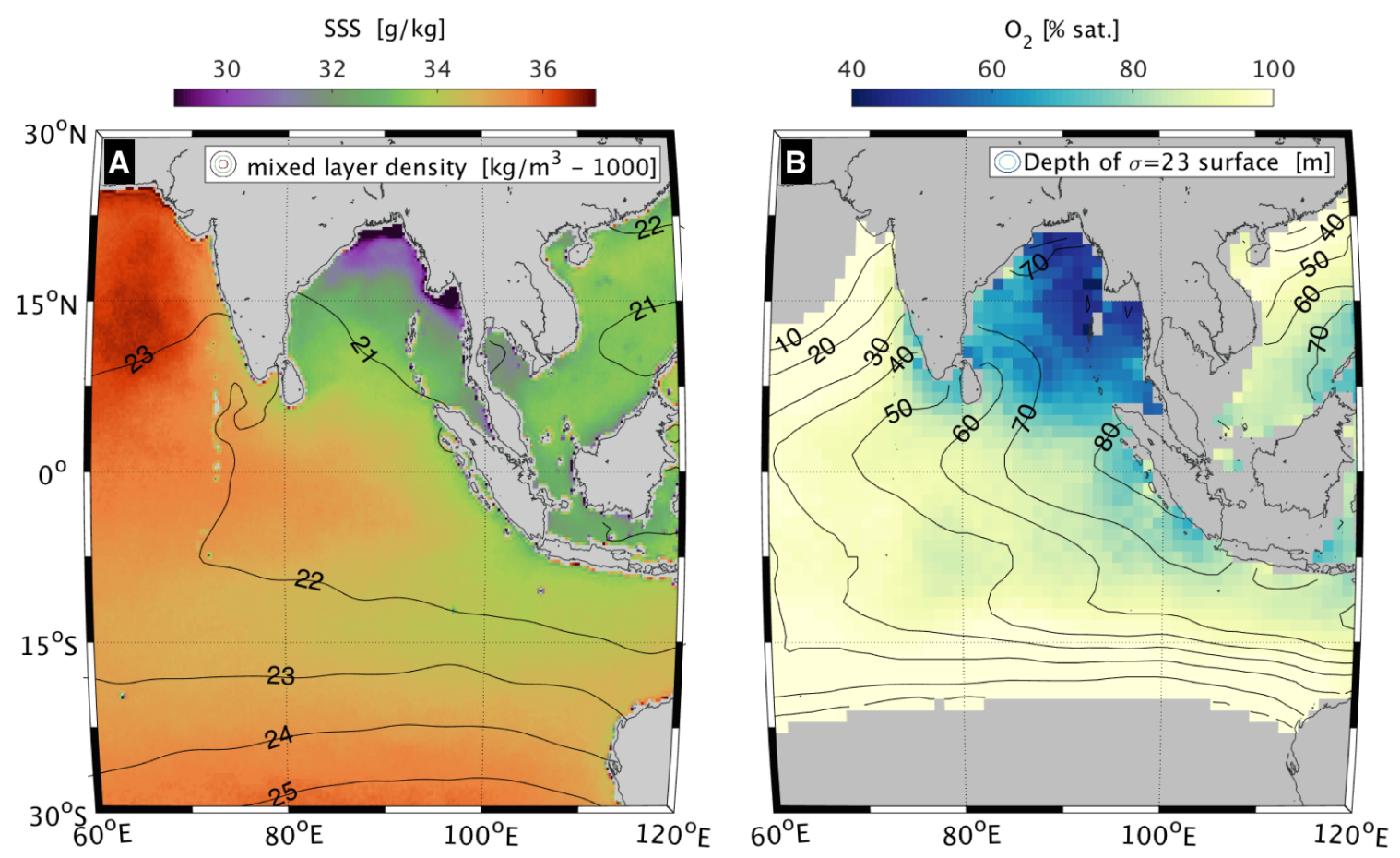

Figure 2-2: Freshwater inputs lower the Bay of Bengal's surface salinity and strongly stratify the upper ocean, insulating a pronounced oxygen minimum zone (OMZ) below about $75 \mathrm{~m}$ depth. (A) Average surface salinity in 2017, measured by SMAP (Meissner et al., 2018). Contoured is climatological annual mixed layer density, from MIMOC (Schmidtko et al., 2013). (B) Climatological oxygen saturation at $1023 \mathrm{~kg} \mathrm{~m}^{-3}$ density $(\sigma=23)$ from WOCE. Contoured is the depth of the $1023 \mathrm{~kg} \mathrm{~m}^{-3}$ surface. At a mean depth of about $75 \mathrm{~m}$ in the Bay of Bengal, this density surface does not regularly outcrop in the bay. Low biological productivity compared to other OMZ regions suggest that the subsurface waters in the Bay of Bengal have long residence-times with little ventilation.

below the surface (Fig.2-2B), which exists even though the Bay of Bengal's surface primary production is not elevated compared to other tropical oceans (supplementary Fig.2-13). Shown in the same figure, oxygen saturation on a particular density surface $\left(1023 \mathrm{~kg} \mathrm{~m}^{-3}\right)$ shows a marked along-isopycnal contrast between the Bay of Bengal and the equatorial Indian Ocean to the south, indicating a relative lack of exchange between the basins and a long residence time of water in the interior of the BoB.

In addition to enhanced vertical salinity gradients above the thermocline, freshwater forcing also creates horizontal gradients in salinity, filling the BoB with both dynamic fronts in density, and passive (along isopycnal) contrasts between different water masses in the pycnocline (Gordon et al., 2016). Climatological sub-surface isopycnal salinity fields are shown on an isopycnal surface for 3 different annual periods during which in-situ observations were 
taken (Fig.2-3). The three expeditions took place in three different seasons: southwesterly winds and rain during the early summer and late summer monsoon marked the cruises from June 6 to 18, 2018, and from August 24 to September 19, 2015, while dry northeasterly winds of the winter monsoon (and one cyclone) prevailed during the first cruise from November 29 to December 112013.
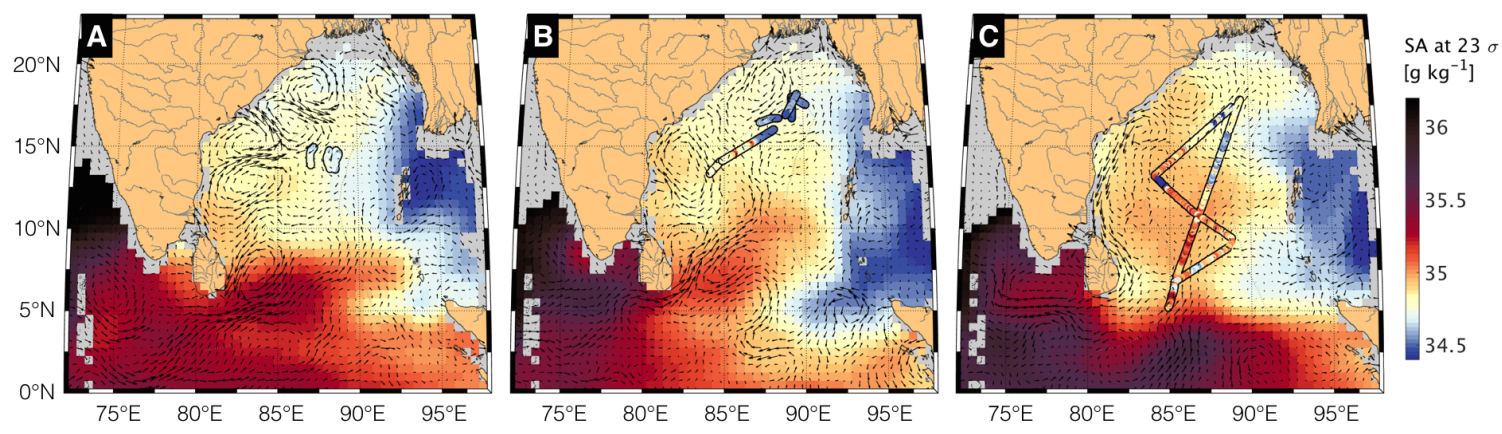

Figure 2-3: The three research expeditions' tracks and the mesoscale currents at the time, along with climatological spice patterns. (A) 6-18th June 2018, (B) 24th August - 20th September 2015, (C) 29th November - 11th December 2013. Background colors show the climatological salinity on the $\sigma=23$ surface for each respective month(s) from MIMOC. The colored dots show the ship-observed salinity at the same density, for all sections longer than $100 \mathrm{~km}$. The black arrows show surface AVISO currents for a particular time period during each cruise. The basin-scale spice gradient is between salty-warm water from the Arabian Sea and relatively fresh-cold water, presumably created by strong internal-tide and bathymetry-driven mixing of fresh surface water in the Andaman Sea. The western side of the Bay of Bengal hosts energetic mesoscale eddies of $O(100) \mathrm{km}$ scale, as well as seasonally reversing boundary currents.

The sub-surface salinity fields from MIMOC, the Monthly Isopycnal \& Mixed-layer Ocean Climatology (Schmidtko et al., 2013), which is based primarily on Argo profiles, reveal the presence of both a salty (and relatively warm) water mass infiltrating from the Arabian Sea, and a fresh (and relatively cold) water mass leaking in from the Andaman Sea through channels in between the chain of Andaman and Nicobar Islands. The monthly climatological fields on the $1023 \mathrm{~kg} \mathrm{~m}^{-3}(\sigma=23)$ density surface exhibit persistent gradients between these end-member water masses. Also shown are mesoscale surface currents during the in-situ observational period (based on AVISO sea surface topography), dominated by eddies that are $O(100) \mathrm{km}$ in horizontal extent.

Along-isopycnal sub-surface salinity variations (compensated by temperature) display both an east-west gradient within the bay, and a north-south gradient in the southern bay (Fig.2-3). The mean monthly patterns of salinity offer insights into time-mean sub-surface 
flows, mirroring the spatial distribution of oxygen along this same density surface. Along the $\sigma=23$ surface at around $70 \mathrm{~m}$ depth, the higher salinity of the equatorial Indian Ocean infiltrates into the Bay of Bengal along its eastern margin, especially later in the summer. This increases the mean salinity and its gradients in the eastern BoB by winter, at which point the mean flow at the southern edge is southbound again. The meeting of distinct water masses in the upper $200 \mathrm{~m}$ of the Bay of Bengal make it an interesting site to study the processes affecting lateral tracer transport both within and below highly stratified ocean layers. The expeditions sampled substantially fresher and saltier water, and sharper gradients, than the climatological values in the interior of the bay.

\subsubsection{Measurement of $T$ and $S$}

The 2013 survey used an Oceanscience UCTD Underway Profiling System (or Underway CTD system, or UCTD), measuring seawater conductivity, temperature, and pressure (Ramachandran et al., 2018). The profiler contains a battery-powered, internally recording CTD, attached with a long line to a tail spool. During the long sections while the ship was underway at $\sim 8 \mathrm{kn}$, the UCTD system was deployed in "free cast" mode. The probe is dropped over the stern and falls nearly vertically while unwinding line from its tail spool. On the deck, a winch pays out line to compensate for the ship's forward travel. Designed to fall through the water at approximately $4 \mathrm{~m} \mathrm{~s}^{-1}$, the probe's $\sim 60 \mathrm{~s}$ fall time was set to reach a target depth of at least $200 \mathrm{~m}$ for all casts. Round-trip time is considerably longer ( $\sim 10 \mathrm{~min})$ due to winch recovery and line re-winding time.

The 2015 and 2018 surveys used the FCTD system ("Fast" Conductivity Temperature Depth), which was developed at Scripps Institution of Oceanography to collect rapid, realtime CTD profiles in the upper $2 \mathrm{~km}$ of the ocean (Pinkel et al., 2012). The subsurface package is comprised of a streamlined profiler with a Seabird (SBE) 49 CTD and associated telemetry and control electronics, affixed to a load-bearing power and signal cable. The profiler is overboarded with a $\sim 10 \mathrm{~m}$ boom to minimize contamination from the ship's wake, and retrieved utilizing a custom designed high speed winch. Winch controls are mainly automated, although watch-standers on deck and in the laboratory monitor the system at all times. Real-time CTD and profiler flight dynamics are collected and analyzed continuously. In the BoB, the FCTD was configured to collect profiles to $200 \mathrm{~m}$ depth while the ship was steaming at 4 knots (2015) and 2 knots (2018). Profiler surface-to-surface round-trip 
time averaged roughly 3 minutes, providing $300 \mathrm{~m}$ (or less) horizontal separation between profiles.

The UCTD and FCTD profiler's raw measurement of temperature and conductivity at 16 $\mathrm{Hz}$ and a fall-rate of approximately 4 and $5 \mathrm{~m} \mathrm{~s}^{-1}$, yields between 3 to 4 samples per meter in the vertical. Before gridding in depth, salinity was calculated by combining the temperature and conductivity profiles with a small temporal offset based on a lagged-correlation for each profile to eliminate salinity spiking artifacts (Thomson and Emery, 2014). A vertically gridded, 1-decibar (approximately $1 \mathrm{~m}$ ) data product was formed by bin averaging, removing outliers with a median filter, and quality controlling each section, with special attention paid to the small fluctuations in salinity and temperature at depth. In several of the sections, the salinity-spiking correction removes abrupt, step-like horizontal changes in salinity between adjacent sections of profiles.

\subsubsection{Hydrography of the Upper $200 \mathrm{~m}$}

Characteristics of the vertical hydrographic profiles measured in the upper $200 \mathrm{~m}$ of the BoB are shown in Fig.2-4. Density stratification, defined as the vertical gradient of buoyancy $\left(N^{2} \equiv-\frac{g}{\rho_{o}} \frac{\partial \rho}{\partial z}\right)$ is strong, sharp, and shallow, with mean peak values of $N^{2} \sim 3 \times 10^{-3} \mathrm{~s}^{-2}$ (buoyancy frequency $N=0.04 \mathrm{~s}^{-1}$ ) at $25 \mathrm{~m}$ depth during Aug.-Sep. 2015, and slightly deeper at $50 \mathrm{~m}$ during both the Nov.-Dec. 2013 and June 2018 surveys. Stratification drops from a peak value of $N^{2} \sim 3 \times 10^{-3} \mathrm{~s}^{-2}$ in the sharpest pycnocline layers to $10^{-4} \mathrm{~s}^{-2}$ at $200 \mathrm{~m}$ depth. Buoyancy frequency $N$ is typically 100 to 1000 times larger than the local Coriolis frequency $f \sim 3 \times 10^{-5} s^{-1}$.

Average $T S$ profiles follow a curve with a halocline transitioning into a deeper thermocline (Fig.2-4B). While at $200 \mathrm{~m}$ depth there is very little $T S$ spread across the three different seasons and years, the surface values show consistent differences between the different seasons. The warmest surface waters were seen in the early summer, the freshest in the later summer monsoon season, and the coldest in the winter season. Most surface mixed layer densities were lighter than $1021 \mathrm{~kg} \mathrm{~m}^{-3}$, and no surface waters denser than $1022 \mathrm{~kg} \mathrm{~m}^{-3}$ were encountered. In between the 1022 and $1024 \mathrm{~kg} \mathrm{~m}^{-3}$ densities, at intermediate depths between approximately $50-100 \mathrm{~m}$, there is considerable along-isopycnal $T-S$ spread. The 2013 survey, which had the largest geographic range, measured both the saltiest (warmest) and freshest (coldest) waters of the entire dataset. The smallest $T S$ variability 

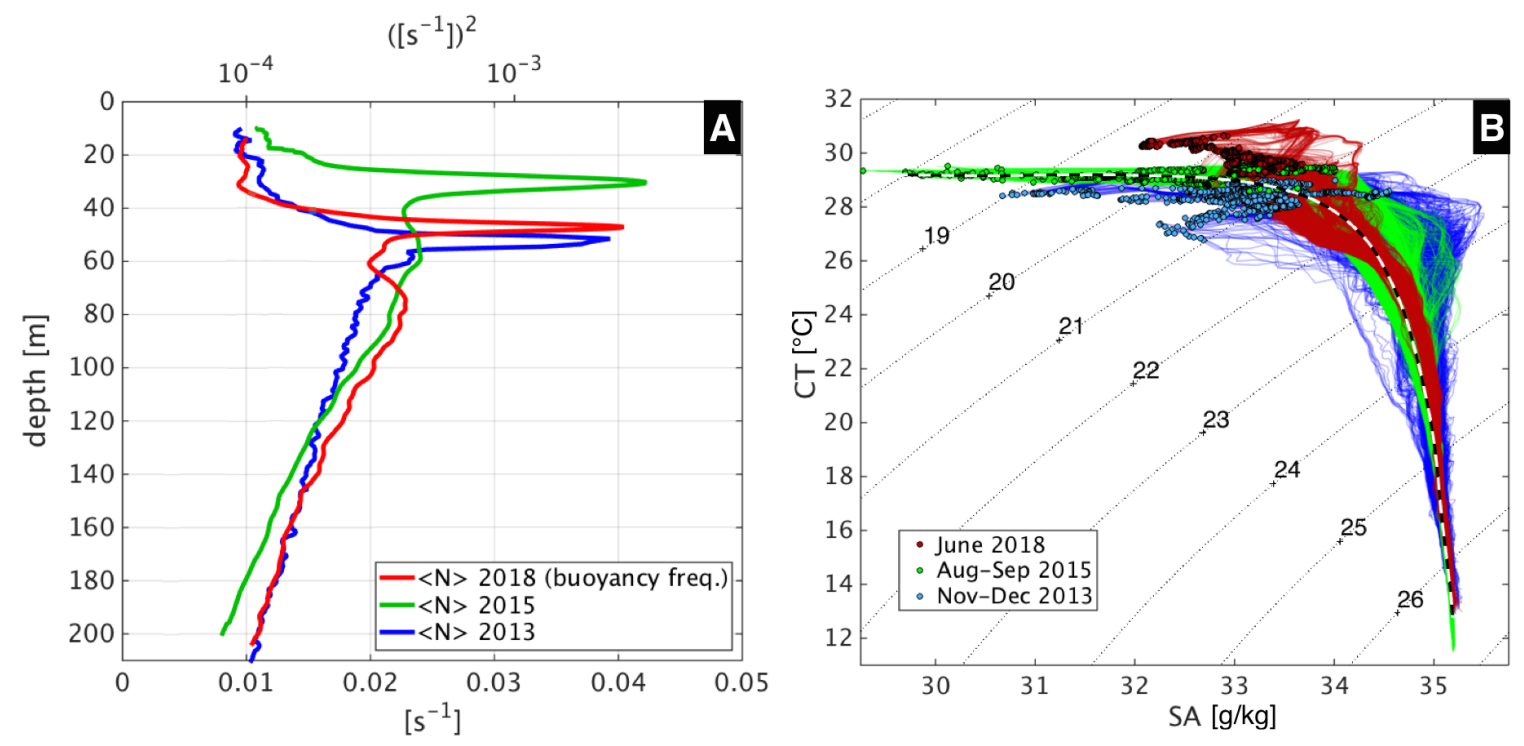

Figure 2-4: The Bay of Bengal's upper ocean is characterized by a shallow sharp halocline above a deeper thermocline creating strong density stratification, and a subsurface layer of enhanced water mass variability. (A) Mean buoyancy frequency $\left(N=\sqrt{\frac{-g}{\rho_{o}} \frac{\partial \rho}{\partial z}}\right)$ profiles from the 3 cruises: June 2018 (red), August-September 2015 (green), Nov-Dec 2013 (blue). Before averaging, the individual buoyancy frequency (i.e. density stratification) profiles are vertically shifted such that their maximum values align at the average depth of peak stratification, to preserve the sharpness of the typical stratification profile. The vertical resolution of $T S$ profiles is at least $1 \mathrm{~m}$. (B) Temperature-Salinity plot of profiles measured on 3 cruises. Circles mark surface mixed layer values, thin dotted contours show potential density referenced to the surface, and the dashed black-white line is a smoothed median TS profile used as the reference for calculating along-isopycnal spice.

was measured during the 2018 cruise, in which shorter ship tracks were sampled.

Water mass anomalies are defined as anomalies of absolute salinity $S$ (or conservative temperature $T$ ) from the average $S$ (or $T$ ) value along isopycnals calculated from all cruises. The salinity anomalies $\Delta S$, scaled by the haline contraction coefficient $\beta$, are equivalent to the temperature anomalies $\Delta T$, scaled by the thermal expansion coefficient $\alpha$, since by definition the anomalies must compensate each other's effect on density. The two components summed together, or equivalently, individually multiplied by two, define water mass or "spice" anomalies. As long as there is a spread in $T S$ values at the same density, water mass variability can be used as a passive tracer of seawater, since buoyancy gradients and dynamics are not affected by the particular values of $T$ and $S$, but only their combined effect on density. Though modified at the surface by input (and loss) of freshwater and heat, once subducted beneath the ML, a water parcel's TS properties are conserved as it 
is stirred on isopycnals, and changed only by interior mixing. Water mass variance forced at large scales cascades to smaller scales and is ultimately dissipated at the 3-D turbulence scale. Furthermore, since we measure the lateral water mass gradients along isopycnals, the signal is unaffected by the heave and vertical strain caused by internal waves.

\subsection{Spectral Analysis Methods}

The vertical profiles of temperature and salinity were processed as follows to compute horizontal wavenumber spectra of water mass anomalies along density surfaces. Profiles of $T$ and $S$ were vertically interpolated onto an evenly spaced density profile with $0.1 \mathrm{~kg} \mathrm{~m}^{-3}$ increments, effectively producing measurements of spice along isopycnal surfaces for each section. The longest section from each cruise is plotted in Fig.2-5, showing isopycnal spice anomalies. Since $S, T$ and spice anomalies along isopycnals are proportional to one another, we need use only one to calculate statistics. We use $S$ to be consistent with some of the previous literature. For each section that was approximately straight and longer than $100 \mathrm{~km}$ (all spice sections shown in Fig.2-14), salinity anomalies along each isopycnal were horizontally linearly interpolated onto a regularly spaced distance grid with spacing smaller than the average profile spacing.

Spectra are calculated along each isopycnal using a Fast Fourier Transform (FFT) of detrended and Hanning-windowed segments. The Fourier coefficients are averaged over neighbouring isopycnals less than $\pm 0.5 \mathrm{~kg} \mathrm{~m}^{-3}$ removed from the target isopycnals of $\sigma=$ $21,22, \ldots, 26$, and further averaged in 10 logarithmically spaced wavenumber bins per decade ( $k$ in cycles per kilometer). For clarity of presentation, and to facilitate the visual differentiation between negative slopes, the plotted variance spectra $\mathcal{P}(k)$ are whitened by multiplying by $4 \pi^{2} k^{2}$, producing gradient spectra $\mathcal{G}(k)$. These steps are shown in Fig.2-6 for the $\sigma=22$ and 25 isopycnals along the longest section from 2015 shown earlier.

Following the procedure described in Appendix A of Klymak et al. (2015), we have also applied a correction for variance lost at high wavenumbers due to finite sampling and horizontal gridding. The correction was determined empirically by making synthetic spatial series of a $k^{-2}\left(k^{0}\right)$ red-noise process, applying exactly the same non-uniform sampling as the profile spacing from the sections, interpolating onto a regular grid, and calculating spectral estimates as described above. These estimates were then compared to spectra 


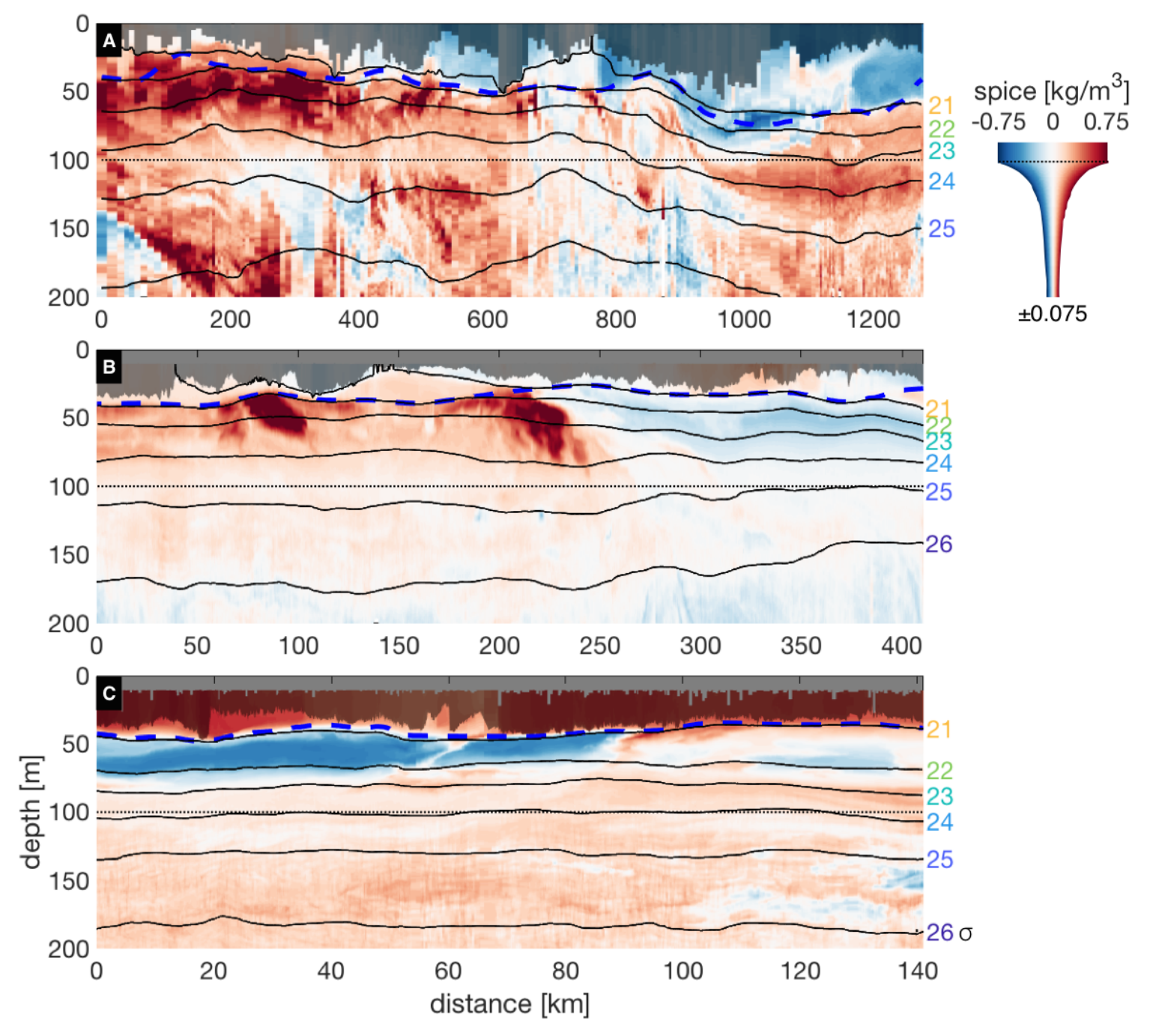

Figure 2-5: Depth vs. distance sections of spice from cruises in the Bay of Bengal in (A) 2013, (B) 2015, and (C) 2018. Note the different horizontal scales. Spice is defined as $\alpha \Delta T+\beta \Delta S$, the along-isopycnal compensated anomaly from the reference $T S$ profile shown in Fig.2-4B. Below $100 \mathrm{~m}$ depth (dotted line), the color values have been enhanced by a factor of up to 10, as indicated in the colorbar, to show the spice anomalies at depth that are much smaller in magnitude than the shallower anomalies. Black lines mark isopycnals 1021 to $1026 \mathrm{~kg} \mathrm{~m}^{-3}$ ( $\sigma=21$ to 26 ) along which we compute the wavenumber spectra of salinity anomalies. Blue dashed line marks the depth of maximum stratification $N^{2}$, gray shaded bars cover the surface mixed layer (ML, defined as the depth with $0.1 \mathrm{~kg} \mathrm{~m}^{-3}$ increase in density from the surface). Below the ML, vertical profiles of spice have been interpolated onto a density profile and then plotted vs. average depth of isopycnals, using a moving-window average along the section, to remove high-frequency isopycnal heave, but retain the large-scale isopycnal slopes and ML structure.

calculated from the full-resolution synthetic spatial series. The roll-off is consistent with the empirical transfer function $\operatorname{sinc}^{2}(\Delta x)$ with $\Delta x$ equal to the mean separation between casts 

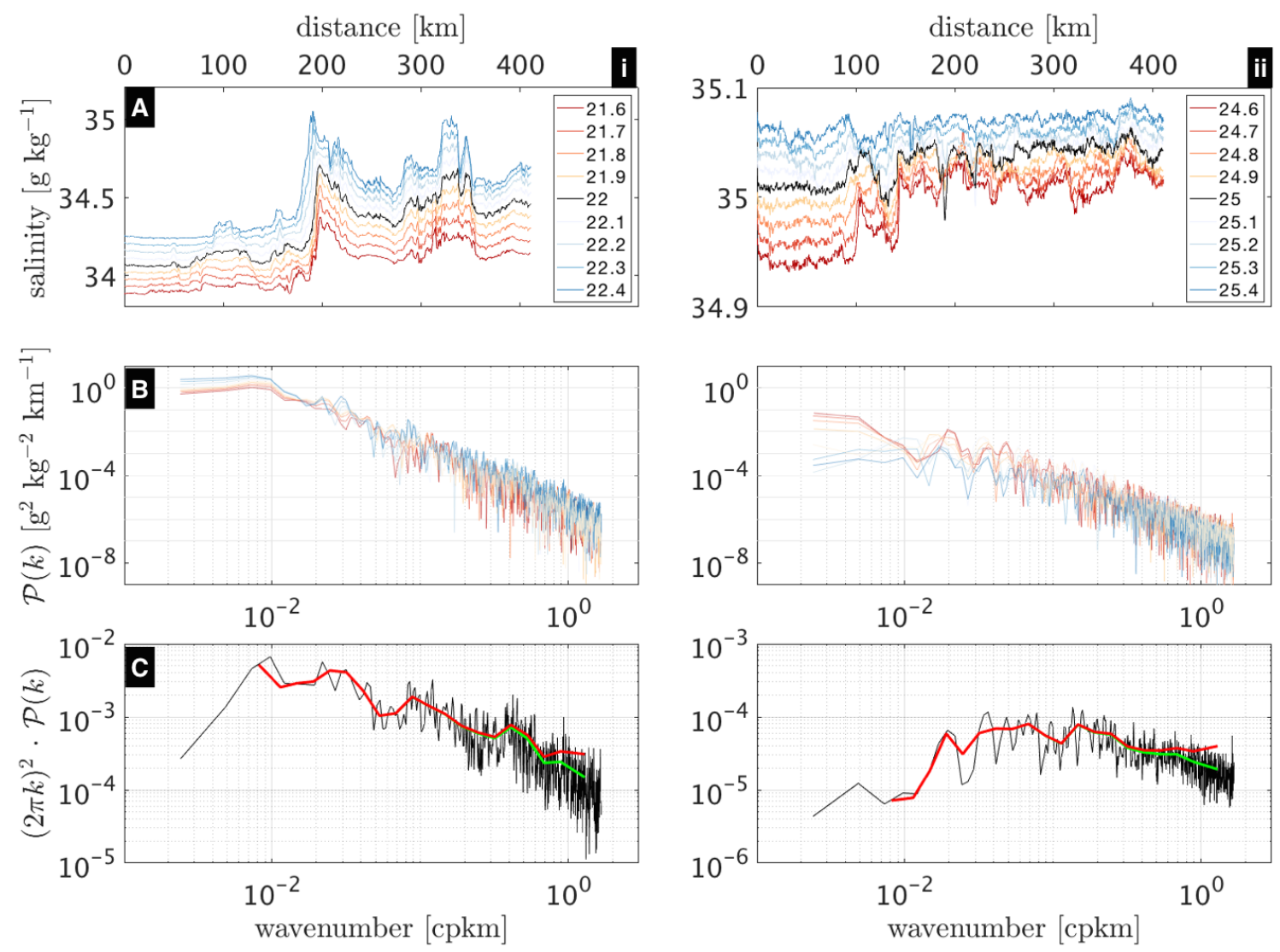

Figure 2-6: Spectral analysis calculations along different isopycnals, (i) $\sigma=22$, (ii) $\sigma=$ 25, for section $\mathrm{F}$ from the 2015 cruise shown in Fig.2-5B. (A) Isopycnal salinity vs. distance, at a range of densities (colored lines) within $\pm 0.4 \mathrm{~kg} \mathrm{~m}^{-3}$ of the target isopycnal (black line). (B) Power spectral density $\mathcal{P}(k)$ for each isopycnal salinity record. (C) Mean $\mathcal{P}(k)$ (black line), multiplied by $4 \pi^{2} k^{2}$, whitened spectrum. This mean spectrum is then bin-averaged in 10 logarithmically spaced bins per frequency decade (green line). To account for highwavenumber roll-off due to limited horizontal sampling resolution $\Delta x$, a correction given by $\operatorname{sinc}^{2}(\Delta x)$ is applied (red line), and the spectral estimate is limited to wavenumbers between $\sim 0.01$ and $\sim 1$ cpkm.

for each section. The correction is perhaps an overestimate for the shallower isopycnals, since it assumes an underlying red-noise $k^{-2}\left(k^{0}\right)$ signal, whereas the observations at the fully resolved wavenumbers reveal an even steeper spectral fall-off of $k^{-3}\left(k^{-1}\right)$.

For the 2013 large-scale survey cruise, with one section over $1000 \mathrm{~km}$ and three sections between $600 \mathrm{~km}$ and $700 \mathrm{~km}$ long, and an average UCTD profile spacing of $3 \mathrm{~km}$, the spectral estimate is confined between wavelengths of $\sim 600 \mathrm{~km}$ and $\sim 10 \mathrm{~km}$. For the 2015 cruise with the longest section over $400 \mathrm{~km}$ long, but the other sections between $100 \mathrm{~km}$ and $200 \mathrm{~km}$ long, and an average FCTD profile spacing of $0.3 \mathrm{~km}$, the spectral estimate is confined between wavelengths of $\sim 100 \mathrm{~km}$ and $\sim 1 \mathrm{~km}$. In contrast, during the 2018 cruise, 
the sections were sampled much more slowly, with $\sim 1 \mathrm{~m} \mathrm{~s}^{-1}(2 \mathrm{kn})$ ship speeds, still at least double the speed of currents below the ML, though of the same order of magnitude. Our sampling speed is $\sim 3$ times faster than the "slow" sampling tested in Rudnick and Cole (2011), which confirmed that spectral estimates from data collected along isopycnals with a slowly-moving platform, such as a glider, are reliable with regards to internal wave aliasing problems, but might be affected by a "Doppler smearing" of features in wavenumber space. Since thermohaline features in the ocean are not frozen, but move during the time it takes a ship to sample them, there is an uncertainty in the true distance between sampled horizontal features. Given the mean current and shear magnitudes relative to the ship speed, measured lengths of features of $O(1-10) \mathrm{km}$ scales are estimated to differ from actual lengths at most by a factor of 2 . This is treated as an uncertainty in wavenumber, but is relatively small given the logarithmic range of scales. Given the slower sampling, the spectral estimate from the 2018 data is confined between wavelengths of $\sim 30 \mathrm{~km}$ and $\sim 1 \mathrm{~km}$. The spectral slopes from the 2018 data are consistent with the spectral slopes in the same wavenumber range from the 2015 data, which were sampled at faster ship speeds.

Within each of the 3 cruise datasets, spectra along the same isopycnals, but in different sections, have broadly similar shapes (Fig.2-7). We thus average them to form a single spectral estimate per target isopycnal for each of the three cruises. The individual and the average isopycnal spectra for each cruise are shown in Fig.2-8B. Furthermore, even though the three hydrographic surveys sampled different seasons, years, and scales, the spectra from the three separate cruises are consistent with each other. They cover a $1 \sim 100 \mathrm{~km}$ range of horizontal wavenumbers, with the 2013 data spanning long wavelengths, the 2018 data spanning short wavelengths, and the 2015 data overlapping in between. There are some changes in variance levels between the years, but the three datasets show the same general depth-dependent trends of decreasing variance levels and shallower spectral slopes with increasing depth.

Given this spectral similarity between the datasets, we combine the individual spectra to form one average spectral estimate for the entire BoB dataset along six density surfaces (Fig.2-9). To combine the individual spectra, they were first normalized by their variance levels at the wavenumber where they overlap $(0.1 \mathrm{cpkm})$, then wavenumber-bin averaged, and then multiplied by the average of their variance levels at $0.1 \mathrm{cpkm}$. These are robust spectral estimates, based on measurements made along 14 sections that cumulatively span 

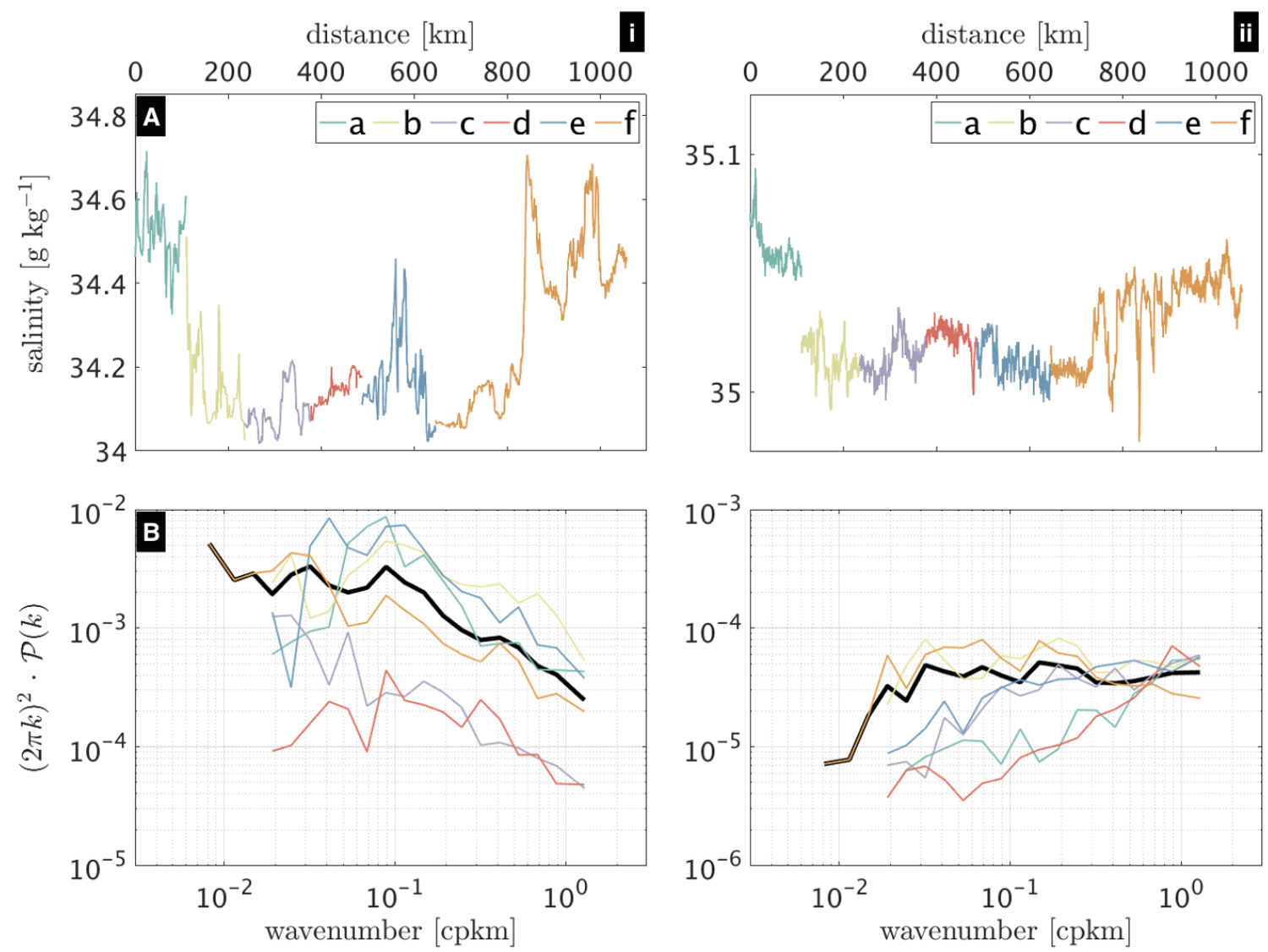

Figure 2-7: Combining spectral estimates from different sections along the same isopycnals: (i) $\sigma=22$, and (ii) $\sigma=25$, during the 2015 cruise. (A) Isopycnal salinity vs. cumulative distance, displaying all sections longer than $100 \mathrm{~km}$ (labeled A to F, shown in Supp. Fig.214). (B) Individual spectral estimates, frequency-bin averaged (colored lines) and the mean (thick black line). The spectra of the individual sections are similar to each other, and the mean is broadly representative of the individual spectral shapes. In calculating the mean, we weighted the individual spectra by the relative length of the sections, for example, the frequency-binned spectra from section $\mathrm{F}$ (orange, $400 \mathrm{~km}$ long) was assigned 4 times the weight the spectra from section A (teal, $100 \mathrm{~km} \mathrm{long).}$

$4864 \mathrm{~km}$ of survey track, equivalent to 50 independent segments of $100 \mathrm{~km}$ length each. Following Klymak et al. (2015), estimates of 90\% confidence bounds are calculated with a chi-squared distribution, with degrees of freedom equal to the number of independent spectral estimates within each wavenumber bin. This is estimated as twice the number of squared Fourier coefficients that are averaged (by averaging over different segments and over adjacent wavenumber bands) to form the estimate within each wavenumber band, reduced by an ad-hoc factor (3) to be conservative. The sum accounts for coefficients from FFT's for all (9) isopycnal layers near each target isopycnal, but reduces this by an 

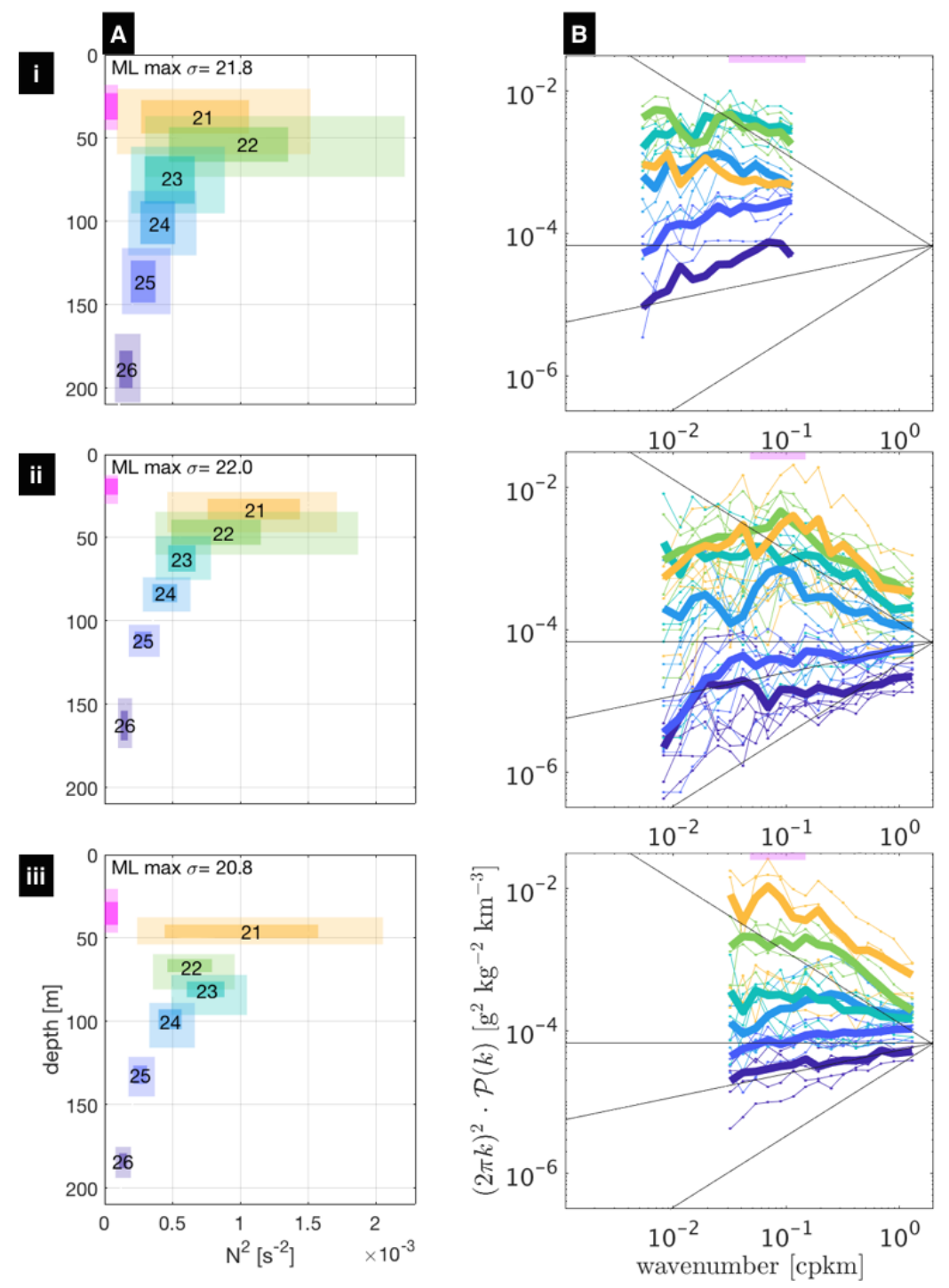

Figure 2-8: Measurements of salinity along isopycnals, and derived statistics of the horizontal scale of tracer gradients, based on hydrographic sections each at least $100 \mathrm{~km}$ long, located as shown in Fig.2-3. (i) Nov-Dec 2013 data, 4 sections, in total 3268 km. (ii) Aug-Sep 2015 data, 6 sections, in total $1057 \mathrm{~km}$. (iii) June 2018 data, 4 sections, in total $539 \mathrm{~km}$. (A) Distribution of depth and stratification $\left(N^{2}\right)$ of isopycnals 21 to $26 \sigma$ (yellow to blue) during the 3 hydrographic surveys. The darker box covers the interquartile range of values, the lighter box covers the 10th to 90 th percentile of values. Similarly shown is the range of depth of the mixed layer (magenta). The maximum ML density encountered in each cruise is noted. (B) Gradient spectra (spectra $\mathcal{P}(k)$ of salinity variance along isopycnals, multiplied by $4 \pi^{2} k^{2}$ ) computed along the respective color-coded isopycnals, averaged for all sections. The average spectral shapes are representative of the individual ones, shown as thin lines. Gray lines mark $\mathcal{P}(k) \sim k^{m}$ power law slopes with $m=-1,-5 / 3,-2$, and -3 (whitened before plotted, like the spectra). Magenta shaded bar at the top marks the scale of predicted surface mixed layer Rossby deformation radii $=N H_{M L} / f$. 
ad-hoc factor (linearly increasing from 2 at the highest wavenumbers to 4 at the lowest wavenumbers) to account for the vertical correlation of anomalies between neighbouring isopycnal layers. The wavenumber-dependence of the factor is chosen in approximation of the results of Klymak et al. (2015), who found a scale-dependent vertical correlation. A visual inspection of salinity anomalies along neighbouring isopycnals (Fig.2-6 A) supports this approach, as large-scale anomalies seem more correlated than small-scale anomalies between nearby isopycnals. Furthermore, the signals of interest in the spectra, such as clear changes of slope, are much larger than the uncertainty estimate. Power law slopes are estimated by a linear fit in log-log space, with the uncertainty in slope dominated by the spectral level uncertainty at small wavenumbers.

\subsection{Results: Tracer Spectra}

The tracer spectra from the three expeditions offer a robust estimate of the variance distribution ranging from $O(100)-O(1) \mathrm{km}$ in the upper pycnocline of the BoB. Both the variance levels and the spectral slopes of variance distributions are depth-dependent, with generally deeper isopycnals containing less variance and less red spectral slopes compared to shallower isopycnals. The salinity variance along isopycnals shallower than $100 \mathrm{~m}$ is most strongly elevated at scales of $10-100 \mathrm{~km}$, where the variance is 2 orders of magnitude greater than the variance along the deepest isopycnal at about $180 \mathrm{~m}$.

However, the elevated variance on the shallower isopycnals $(<100 \mathrm{~m})$ falls off steeply below $10 \mathrm{~km}$ scales, exhibiting power laws $\mathcal{P}(k) \sim k^{-2.9 \pm 0.2}\left(\mathcal{G}(k) \sim k^{-0.9 \pm 0.2}\right)$ at these length scales (Fig.2-9). At $O(1) \mathrm{km}$ scales, the variance is only about 1 order of magnitude greater than the variance at the same scale along the deepest isopycnals. The scale at which this change in spectral slope occurs is $O(10) \mathrm{km}$, and coincides with the local Rossby radius of deformation in the surface mixed layer $N H_{M L} / f$ (Callies et al., 2016), where we estimate $N \sim 0.01 \mathrm{~s}^{-1}$ and $H_{M L} \sim 30-50 \mathrm{~m}$ from the mean profiles in Fig.2-4B. $f$ ranges from $1 \times 10^{-5}$ to $5 \times 10^{-5} \mathrm{~s}^{-1}$ depending on the latitude of the cruise track.

The steepening of the spectrum is seen both along the $\sigma=21$ and 22 isopycnals, which occasionally outcrop into the ML or into the weakly stratified layers in between the peak stratification depth and the ML base, as well as along the $\sigma=23$ isopycnal, which never outcropped into the ML, and was always below the peak stratification depth in all the cruise 


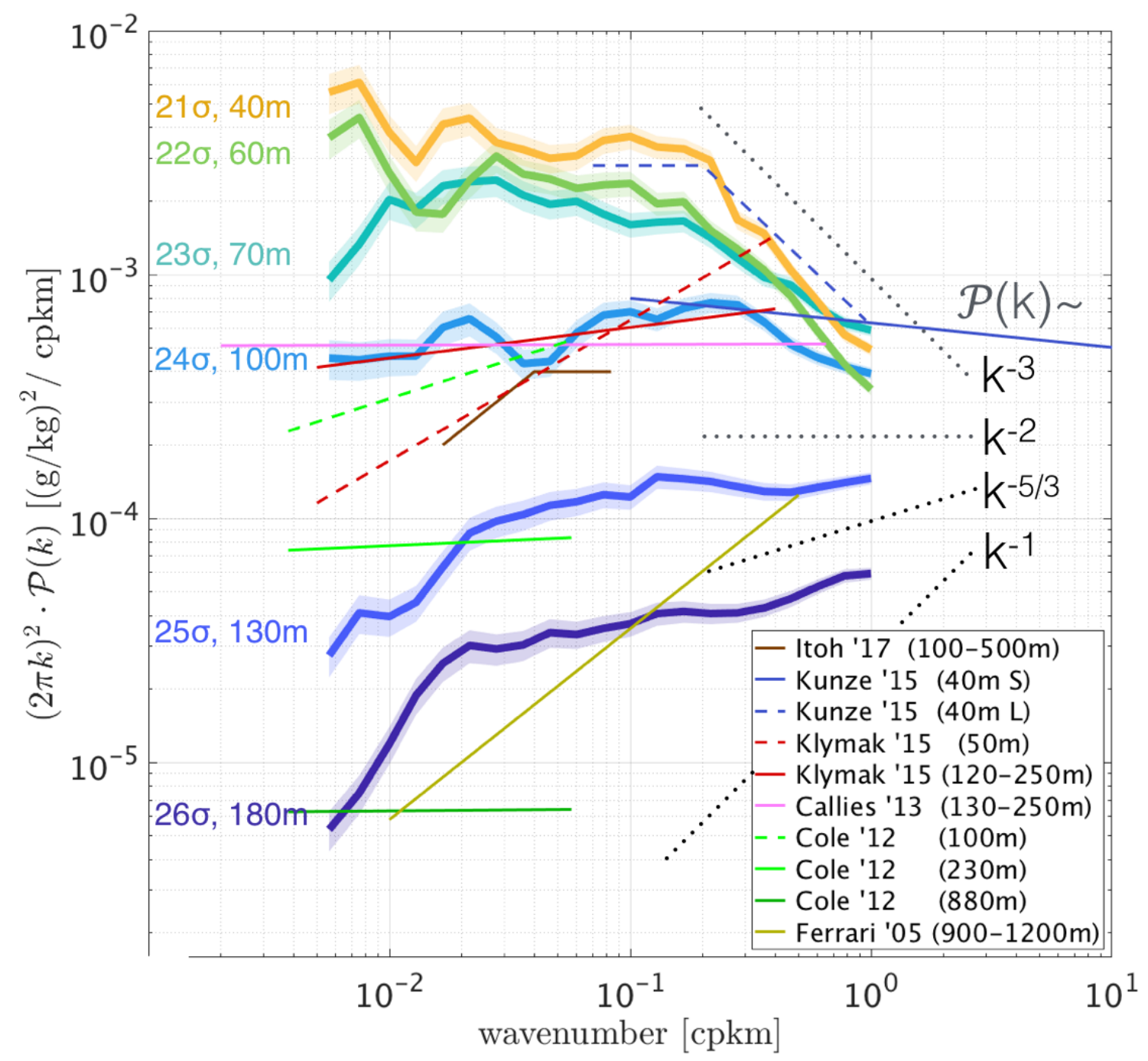

Figure 2-9: Comparison of horizontal wavenumber variance spectra for salinity anomalies along isopycnals at different mean depths, from the Bay of Bengal (shaded areas represent confidence bound estimates as described in text). Thin straight lines depict spectra from other published studies: the CUGN project in the California Current System (Itoh and Rudnick, 2017), the LatMix project in the Atlantic's Sargasso Sea (Kunze et al., 2015) and the NATRE project in the subtropical Atlantic (Ferrari and Polzin, 2005b), the Line P and IWAP cruises (Klymak et al., 2015), the Spice dataset (Callies and Ferrari, 2013), and data from gliders (Cole and Rudnick, 2012) in the subtropical and northern Pacific. Lines are graphical best-fits of the slopes, converted to salinity anomaly spectra in wavenumber $k$ (cycles per kilometer) multiplied by $4 \pi^{2} k^{2}$. Dotted gray lines mark the whitened slopes of variance spectra $\mathcal{P}(k) \sim k^{m}$ with power laws of $m=-1$ (QG stirring), $-5 / 3$ (SQG / Obukhov-Corrsin ), -2 (frontogenesis), and the observed decay of -3 of the shallow BoB spectra below $O(10) \mathrm{km}$.

observations (Fig.2-8A). The $\sigma=21$ and 22 isopycnals are on average in the layer of maximum stratification forming the lower boundary of the ML (mean depths are approximately 
$40 \mathrm{~m}$ and $60 \mathrm{~m}$, mean $N^{2} \sim 1 \times 10^{-3} \mathrm{~s}^{-2}$ ). The $\sigma=23,24,25$ and 26 isopycnals are always in the interior pycnocline layers (mean depths are 70,100,130, $180 \mathrm{~m}$, respectively, mean $N^{2} \sim 0.1$ to $\left.0.5 \times 10^{-3} \mathrm{~s}^{-2}\right)$.

Salinity variance is in general highest along the shallowest isopycnal that is located within or below the peak stratification. Salinity variance is reduced along the shallowest isopycnal $(\sigma=21)$ when it is located in weakly stratified layers above the peak stratification, such as during some of the 2013 and 2015 section. This indicates that these weakly stratified layers are remnant mixed layers containing signatures of surface boundary forcing and variance dissipation.

A qualitative assessment by eye of the spice anomaly or salinity variance on isopycnals (Fig.2-5, Fig.2-6A, Fig.2-7A) is consistent with the spectra that are calculated. For example, the salinity along the shallower isopycnals in the $100 \mathrm{~km}$ long transects of the 2018 data has smaller-scale fluctuations of lesser magnitude than the large-scale variability seen in the longer sections from 2013 (i.e. a red gradient spectrum). Lastly, to complement the spectral analysis, the isopycnal salinity measurements were also analyzed using a wavelet decomposition. The results again show the same scale-dependence of variance, with a lack of variance at $1-10 \mathrm{~km}$ scales relative to $10-100 \mathrm{~km}$ scales along the shallower isopycnals (supplementary Fig.2-15).

\subsection{Discussion}

This study presents a robust set of passive tracer spectra from the relatively sparsely sampled Indian Ocean (Fig.2-10), finding depth-dependent and scale-dependent spectral slopes that are in part inconsistent with current theories of tracer stirring by ocean currents. We compare our results with the few other published studies of tracer spectra in the world oceans and discuss differences in environments and the potential role of submesoscale ocean dynamics that could explain the differences in the tracer spectra.

\subsubsection{Contextualizing the Bay of Bengal Study}

There have been several studies in the Pacific and two in the Atlantic Ocean of the statistics of spatial distributions of spice, or compensated salinity or temperature anomalies along an isopycnal. We briefly summarize them here, and compare linear fits of their spectral slopes 


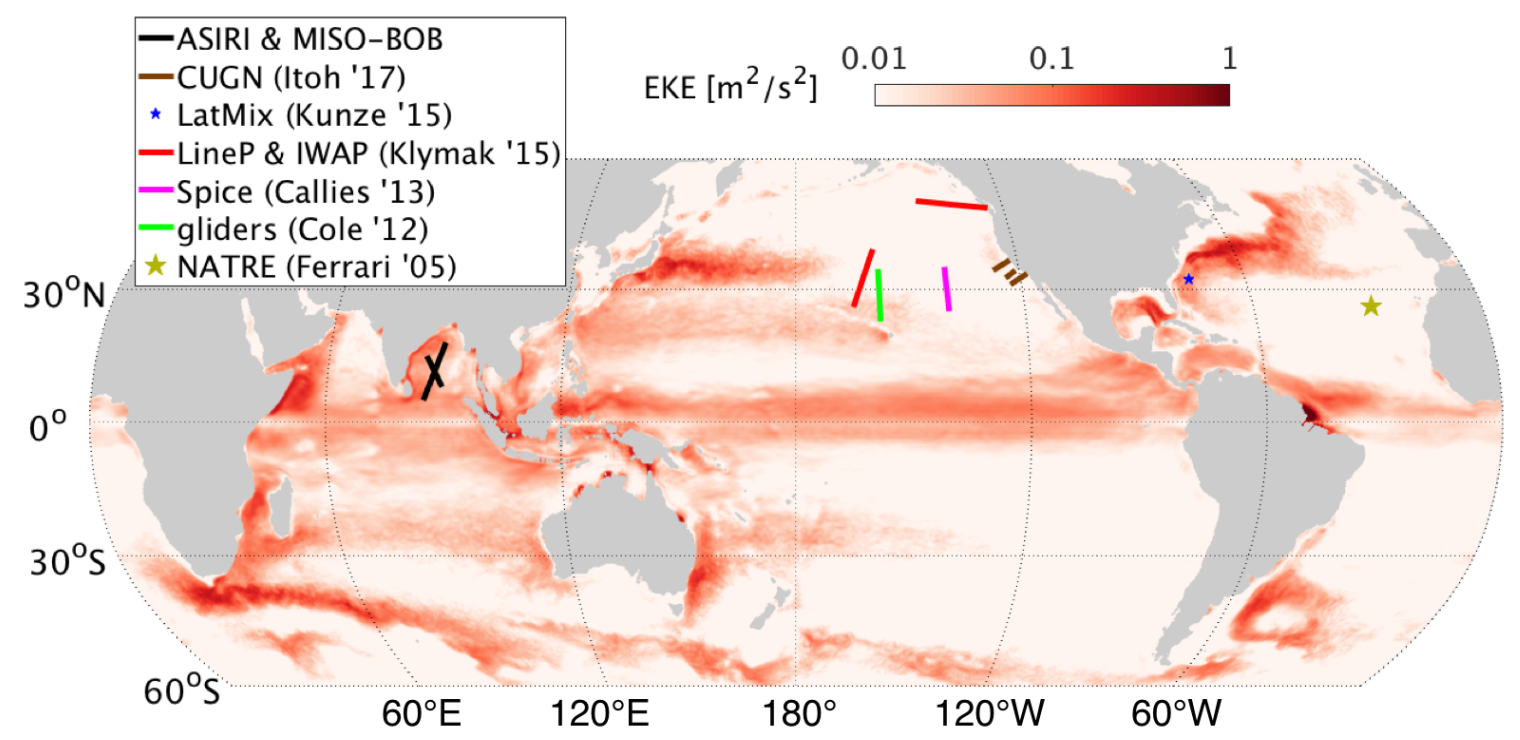

Figure 2-10: Global map of eddy kinetic energy $=\frac{1}{2}\left(u^{\prime 2}+v^{\prime 2}\right)$ based on daily $0.25^{\circ}$ fields from the Global Ocean Gridded SSALTO/DUACS Sea Surface Height L4 product averaged from 2013 to 2017. Colored lines and stars show the location of studies reporting alongisopycnal salinity or temperature spectra, shown in Fig.2-9. Note that only the LatMix study in the Western Atlantic (blue star) was located in a similarly energetic region as the Bay of Bengal (black line).

with our observations in Fig.2-9.

Analysis of observations from the towed instruments in the 'Spice' expedition (Callies and Ferrari, 2013) and from repeated glider sections (Cole and Rudnick, 2012) in the subtropical North Pacific at depths between 100 and 800 m, consistently found isopycnal tracer spectra with $k^{-2}\left(k^{0}\right)$ behavior at $\sim 10-100 \mathrm{~km}$ scale wavelengths (Fig. 2-9). This depthindependent power law is inconsistent with both interior QG theory that predicts spectra to follow $k^{-1}\left(k^{+1}\right)$, as well as surface-QG modes that would predict steeper spectra below the surface.

Another recent analysis (Klymak et al., 2015) of profiler observations in the Gulf of Alaska ('Line P') and undulating towed measurements in the subtropical Northern Pacific ('IWAP') also found tracer spectra between 10-100 km scales to be inconsistent with QG/SQG theory. They instead found power laws usually between $k^{-1.5}\left(k^{0.5}\right)$ and $k^{-1.7}$ $\left(k^{0.3}\right)$, and reddening towards $k^{-2}\left(k^{0}\right)$ along the deepest isopycnals at around $150 \mathrm{~m}$ along Line P. While finding less red spectra than the previous studies, the observed reddening with depth trend is exactly opposite to the expected decay of SGQ modes and increasing dominance of interior-QG modes with increasing depth. 
A wavelet analysis of glider data ('CUGN') from the upper $500 \mathrm{~m}$ of the California Current System, resolving spectra over the narrow wavelength range of $12-60 \mathrm{~km}$, found a change in slope with scale in their isopycnal salinity gradient spectra (Itoh and Rudnick, 2017). On average, across the seasons and inshore/offshore regions, they estimate a $k^{-1}$ $\left(k^{+1}\right)$ slope in their "mesoscale" lower half range, and a $k^{-2}\left(k^{0}\right)$ slope in their "submesoscale" upper half range. They interpret the flat part of the spectrum, at scales smaller than the baroclinic Rossby radius, as the signature of sharp fronts.

A recent study (McCaffrey et al., 2015) applied a novel technique to the global Argo profile dataset, calculating salinity structure functions from scattered profiles instead of spectra along transects. They found equivalent isopycnal tracer spectra power laws of $k^{-1.7}$ $\left(k^{0.3}\right)$ between 10-100 km scales, but with little dependence on depth in the upper $2000 \mathrm{~m}$. Their results are broadly consistent with the other mesoscale studies.

In the subtropical Atlantic Ocean, the 'NATRE' study (Ferrari and Polzin, 2005a) calculated structure functions from TS observations between 900 and $1200 \mathrm{~m}$ depth, finding equivalent isopycnal tracer spectra power laws of $k^{-1.2}\left(k^{0.8}\right)$ between $2-100 \mathrm{~km}$ scales. Extending to smaller scales, the 'LatMix' study (Kunze et al., 2015) in the North Atlantic's Sargasso Sea found tracer spectral slopes again approximately equal to $k^{-2}\left(k^{0}\right)$ over wavelengths ranging from $10 \mathrm{~km}$ to $0.03 \mathrm{~km}$ in the seasonal pycnocline between $20-60 \mathrm{~m}$ depth. However, measurements along a larger survey track (referred to as the MVP and Triaxus surveys) that resolved wavelengths between 10 and $1 \mathrm{~km}$, found very red spectra estimated to lie between $k^{-3}\left(k^{-1}\right)$ and $k^{-2.5}\left(k^{-0.5}\right)$ in the 5 to $1.4 \mathrm{~km}$ wavelength band. Spanning less than a decade of wavenumbers, this estimate was deemed less robust than the main results of the study.

In summary, five of the six published studies found tracer gradient spectra with power laws close to $k^{-2}\left(k^{0}\right)$, inconsistent with QG predictions, but consistent with ageostrophic frontogenesis theory. The power law slopes were generally also independent of depth, or in some cases became less red towards shallower depths, in contrast to the SQG or surfacefrontogenesis predictions of redder slopes near the surface. An exception is reported in the LatMix dataset, which found a red gradient spectral slope between 5 and $1.4 \mathrm{~km}$ wavelengths, with the same $k^{-3}\left(k^{-1}\right)$ power law that we report along shallow isopycnals in the BoB. Perhaps it is notable that both the BoB and the LatMix studies are in regions with elevated frontogenetic potential at the surface due to an energetic mesoscale, compared to the studies 
from the subtropical and northern Pacific. The BoB has a seasonal western boundary current that stands out in the climatological eddy kinetic energy (EKE) map (Fig.2-10), with elevated EKE in the central BoB as well. The present study is also at lower latitudes (5$18^{\circ} \mathrm{N}$ ) than all the above cited studies of along-isopycnal tracer spectra (all located between $\left.23-50^{\circ} \mathrm{N}\right)$. The present region's smaller Coriolis parameter leads to longer inertial periods and larger deformation scales.

\subsubsection{Interpreting Bay of Bengal Spectra}

The classical interpretation of wavenumber spectra of a passive tracer in the ocean interior assumes an injection (source) of tracer variance at large scales (small wavenumbers), a downscale cascade, and a sink of variance at small scales (large wavenumbers). If the statistics averaged over a large enough region are stationary, the variance level at any particular scale in between the forcing and the dissipative scales, depends on how efficiently gradients are strained into smaller features by the 2-dimensional turbulence, and the dissipation rate of tracer variance through 3-dimensional turbulent mixing. Tracer gradients in the interior can also retain signals from external forcing such as air-sea fluxes acting on the surface ML that are then re-stratified and entrained into the interior. Destruction of variance only occurs at the scale of 3-dimensional isotropic turbulent mixing, which can act only on gradients at that scale. Within this framework, the slopes of isopycnal salinity spectra in the upper $200 \mathrm{~m}$ of the BoB at scales between 1-100 km tell a depth-dependent story with three parts.

First, at depths of 150-200 m $(\sigma=26)$, the tracer variance level decays with increasing wavenumber following an approximately $k^{-1.7 \pm 0.1}\left(k^{0.3 \pm 0.1}\right)$ power law. This is in agreement with theories of stratified turbulence, which predict tracer variance distributions follow the Obukhov-Corrsin spectrum $\mathcal{P}(k)=C \cdot \chi \cdot \varepsilon^{-1 / 3} \cdot k^{-5 / 3}$, where $\chi$ is the turbulent temperature flux rate or dissipation rate, $\varepsilon$ the turbulent energy dissipation rate, and $C \approx 0.5$ is an empirical constant (Brethouwer and Lindborg, 2008). Fitting the theoretical spectrum to observed variance levels, and assuming $\varepsilon$ is between $10^{-10}$ and $10^{-8} \mathrm{~W} \mathrm{~kg}^{-1}$, the temperature variance flux rate $\chi$ is predicted to be between $10^{-10}$ and $10^{-9} \mathrm{~W} \mathrm{~kg}^{-1}$. The observed spectral slope is also in agreement with predictions for tracer spectra stirred by two-dimensional geostrophic turbulence in an inverse energy cascade range above the stirring scale (Vallis, 2017). However, it is unlikely that the dominant stirring at these depths is by motions or eddies of $O(1) \mathrm{km}$ or smaller; instead the deeper currents are due to the mesoscale eddy 
field. The slope is also in agreement with SQG predictions, which would necessitate surface modes to penetrate $150-200 \mathrm{~m}$ to the $\sigma=26$ isopycnal layer, even though this depth is clearly below the peak pycnocline and insulated from surface boundary forcing. Estimating the penetration depth $H$ by $\frac{f}{N} \cdot L \sim \frac{3 \cdot 10^{-3}}{0.02} \cdot L, 10 \mathrm{~km}$ wide eddies would penetrate only $20 \mathrm{~m}$, while $100 \mathrm{~km}$ eddies do reach $200 \mathrm{~m}$.

Next, at shallower depths of about 100-130 m ( $\sigma$ 24-25), tracer variance spectra steepen to approximately $k^{-1.9 \pm 0.1}\left(k^{0.1 \pm 0.1}\right)$ power laws, inconsistent with interior QG, somewhat less inconsistent with SQG or stratified turbulence predictions under idealised conditions, but consistent with other observational studies. It potentially indicates that mesoscale frontogenesis and ageostrophic dynamics cascade variance more rapidly than predicted by QG dynamics in the pycnocline (Klein et al., 1998). The redder slope at this depth relative to the deeper $(\sigma=26)$ spectrum is consistent with the notion that more small-scale stirring and straining increases the rate of downscale variance flux more at smaller scales along the tracer cascade, steepening the spectrum, and that ageostrophic small-scale motions attenuate over a shallower depth than geostrophic mesoscale currents.

Finally, at shallow depths of around 40 to $70 \mathrm{~m}(\sigma 21-23)$, the spectra also show an approximately $k^{-2.0 \pm 0.2}\left(k^{0.0 \pm 0.2}\right)$ power law above $O(10) \mathrm{km}$ scales, but an unexpected finding is that they steepen to $k^{-2.9 \pm 0.2}\left(k^{-0.9 \pm 0.2}\right)$ below $O(10) \mathrm{km}$ scales. On isopycnal surfaces that are both above and within the depth of strongest stratification ( $\sigma 21-22)$, and just beneath $(\sigma 23)$, the change in slope at $O(10) \mathrm{km}$ scales suggests a change in dominant dynamics around the ML Rossby deformation radius $\left(\sim N H_{M L} / f\right)$. Spectral analysis does not identify what process is responsible for the steeper fall-off in tracer variance at scales below $O(10) \mathrm{km}$ and at depths $<100 \mathrm{~m}$, or 3 to 4 times the ML depth.

While the literature regularly compares spectral observations with theory, the agreement or disagreement of tracer spectral slopes with theoretical predictions should be interpreted carefully. Predictions are made using idealized assumptions, such as homogeneity and scaleseparation, which allows an inertial range to form. These idealized conditions are unlikely to adequately represent the real conditions in the $\mathrm{BoB}$, where for example kinetic energy might be input at various scales by ML instabilities and atmospheric forcing. More significant than observations of particular slopes are changes in spectral slopes, at particular scales, indicating a change in the dynamics of the variance cascade. The observed steepening of the tracer variance spectra around scales of $10 \mathrm{~km}$ indicates a change in the rate-limiting 
process that transfers variance downscale.

\subsubsection{Potential Explanations}

Might the unusual spectral slope of tracer variance observed along the shallower isopycnals simply be the signature of water mass anomalies subducted or injected into the interior from the surface ML? This explanation by itself seems unlikely, since we observe not an excess, but a lack, of variance at smaller scales. Such reduced levels of variance at relatively smaller $O(1) \mathrm{km}$ scales should be temporary, given the relative excess of variance present at larger $O(10-100) \mathrm{km}$ scales that could be cascaded down-scale. We expect a downscale tracer variance cascade, whether described by QG or ageostrophic theory, to transfer variance across scales (from large to smaller spatial scales), within the timescale of the background mesoscale stirring and straining, which is of $O(10)$ days, given the mean EKE in the BoB of $O(0.1) \mathrm{m}^{2} \mathrm{~s}^{-2}$ and mesoscale eddies of $\mathrm{O}(100) \mathrm{km}$ scale. Yet low oxygen levels (Fig.2-2B) at the $\sigma=23$ isopycnal, which never outcropped in the BoB observations, suggest that water mass anomalies within this layer have residence timescales below the surface ML considerably longer than the timescale of stirring. Thus, if the deficit of 1-km-scale tracer variance along the $\sigma=23$ isopycnal is a remnant signature of mixing in the surface ML prior to watermass subduction, the small-scale variance should be replenished by a downscale variance cascade in the time since the water layer was last ventilated. The fact that small-scale variance is 'missing', in layers that have been insulated from surface-forced mixing for a relatively long time, calls for a different explanation.

We are left with two categories of potential explanations, schematically represented in Fig.2-11, and further discussed below. The observed steepening of tracer spectra below $10 \mathrm{~km}$ is either caused by (mixing) processes actively decreasing variance at scales below $10 \mathrm{~km}$, or by a suppression in the downscale spectral flux of variance below $10 \mathrm{~km}$ scales.

\section{Enhanced Horizontal Mixing at Scales below $10 \mathrm{~km}$}

We propose two candidates for adding effective mixing, which are not mutually exclusive, and could enhance tracer variance diffusion within the pycnocline and explain the steep decay of variance below $O(10) \mathrm{km}$. One is submesoscale frontal instabilities and associated submesoscale circulations that penetrate below the ML, while another is shear dispersion powered by near-inertial generated waves (NIW) with very small vertical wavelengths in the 


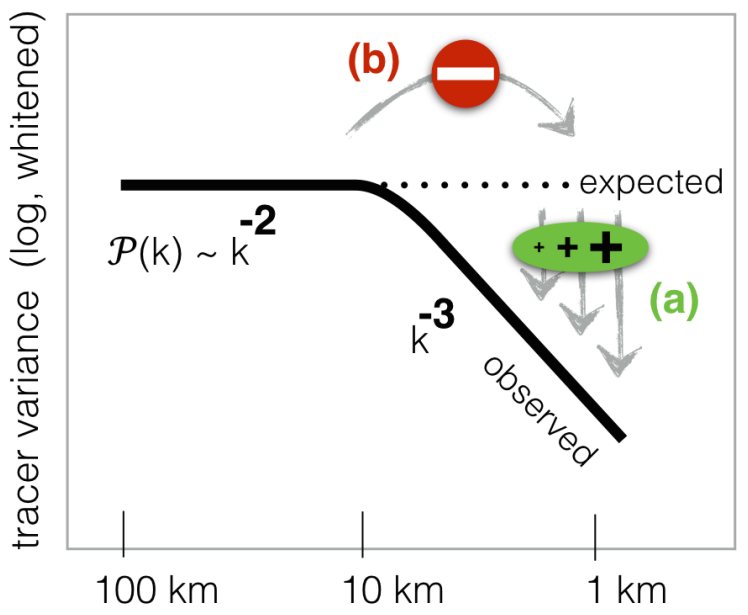

Figure 2-11: Representation of the observed change in spectral slope of spice variance $\mathcal{P}(k)$ (whitened) along the shallower isopycnals in the BoB, idealized from Fig.2-9. Given the variance present at scales $O(10) \mathrm{km}$, we expect at least the same (whitened) variance level to be seen at smaller scales (dotted line), given any of the theories of 2-dimensional stirring in a statistical steady-state. Two potential explanations are suggested for the increasing deficit of tracer variance below $10 \mathrm{~km}$ scales. Either (a) the $O(1) \mathrm{km}$ horizontal variance is destroyed by vertical mixing that is effectively felt at this horizontal scale, which is much larger than the 3-dimensional isotropic mixing scale. Potential candidates are shear dispersion or submesoscale instabilities. Or (b) the down-scale cascade of variance from larger scales is inhibited beyond $\sim 10 \mathrm{~km}$ scales in the Bay of Bengal. A potential reason could be coherence between spice anomalies and dynamic anomalies, such that spice spectral distributions do not reflect stirring by homogeneous macro-turbulence of an independent, stochastically distributed tracer, but rather the distribution of a dynamically relevant property.

strongly stratified pycnocline. Both explanations would need to produce spice patterns with large aspect ratios (long and thin), such that turbulent vertical mixing effectively removes variance at submesoscales (Smith and Ferrari, 2009).

Submesoscale instabilities convert the potential energy of lateral buoyancy gradients in the ML into kinetic energy, and through interactions with the mesoscale strain field, can transfer energy from the mesoscale to submeso- and dissipative scales (McWilliams, 2016). The surface ML of the BoB is characterized by strong density fronts with buoyancy gradients at least one order of magnitude stronger at scales of $O(1) \mathrm{km}$ compared to the mesoscale buoyancy gradients at scales $O(10-100) \mathrm{km}(\mathbf{F i g . 2 - 1 2})$. This ratio is calculated by comparing the largest magnitudes ( 1 and 0.1 percentile) of density gradients in spatial transects smoothed by low-pass filters (3rd order Butterworth) with cutoff wavenumbers ranging from equivalent wavelengths of 0.5 to $50 \mathrm{~km}$. Signatures of submesoscale frontal processes at $O(1-10) \mathrm{km}$ scales have been observed in the BoB (Ramachandran et al., 2018). 
ML eddies generated from baroclinic instabilities can also penetrate below the ML into the pycnocline, stirring water masses along isopycnals (Badin et al., 2011) and enhancing the effective lateral diffusivity at submesoscales.

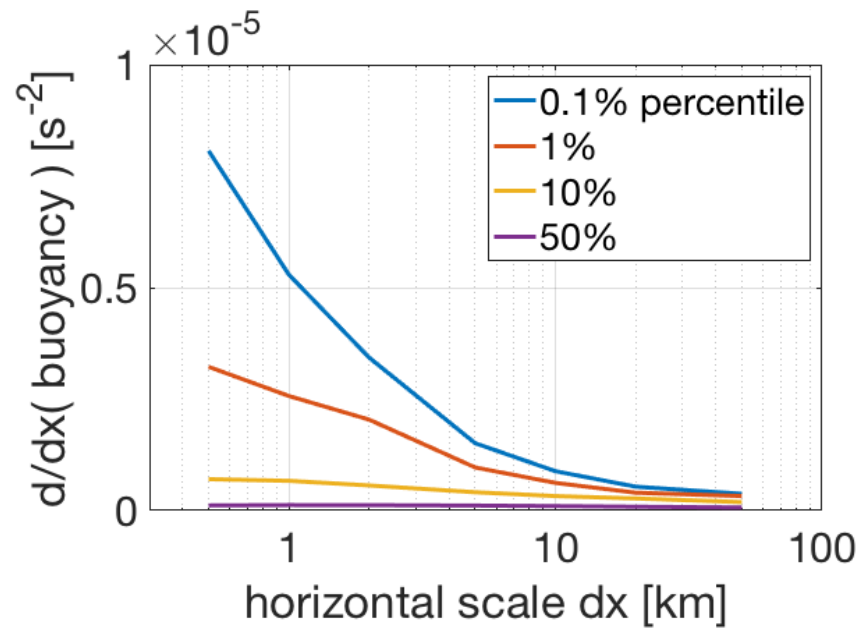

Figure 2-12: The Bay of Bengal's surface mixed layer contains strong submesoscale density fronts. Magnitudes of buoyancy gradients $\left|\frac{g}{\rho_{o}} \frac{d}{d x} \rho\right|$ are calculated along the horizontal sections longer than $100 \mathrm{~km}$ from the 2015 survey, and low-pass filtered (3rd order Butterworth) with increasingly larger cut-off wavelengths. Colored lines show the top percentiles $(0.1,1,10$, and $50 \%$ ) of the buoyancy gradients, showing that the strongest fronts have widths below $O(1) \mathrm{km}$, and are at least one order of magnitude stronger than mesoscale gradients.

The observed tracer variance fall-off below $10 \mathrm{~km}$ scales along isopycnals that lie just beneath the ML, could be caused by enhanced stirring by submesoscale instabilities that are active within the mixed layer, powered by strong freshwater-controlled potential energy gradients or other sources of flow instabilities. The extra submesoscale stirring needs to act only at scales smaller than $O(10) \mathrm{km}$, reducing variance in the $O(1) \mathrm{km}$ band. The energy input by such submesoscale instabilities would invalidate the assumption of an inertial range by the theoretical slope predictions, and complicate interpretations of spectral slopes as related to rates of variance transfer.

The spectral fall-off could potentially also be due to shear dispersion enhancing effective along-isopycnal diffusivity. Internal-wave shear dispersion allows weak turbulent mixing at small scales to effectively diffuse lateral gradients at larger scales much more quickly than expected (Young et al., 1982). A sheared flow, whether or not reversible, tilts tracer isopleths to enhance the cross-diapycnal area of a water mass anomaly compared to its lateral (alongisopycnal) dimension, and amplify the weak but widespread vertical diffusion $K_{v}$ into a much greater lateral diffusion $K_{h} \sim\left(V_{z} / f\right)^{2} K_{v}$, where $V_{z}$ is the average vertical shear magnitude 
(Shcherbina et al., 2015).

Observations in the BoB reveal strongly sheared upper ocean currents dominated by shear layers of $O(10) \mathrm{m}$ thickness, with an average vertical shear of magnitude $0.01 \mathrm{~s}^{-1}$ (Lucas et al., 2016). Given the small average Coriolis parameter in the BoB of $f=3 \times 10^{-5}$ $\mathrm{s}^{-1}$, the effective isopycnal diffusivity is enhanced by a factor of $10^{5}$ over the diapycnal diffusivity. However, observations in the BoB show weak turbulence below the ML, with median values of the diapycnal diffusivity $K_{v}=10^{-6} \mathrm{~m}^{2} \mathrm{~s}^{-1}$ in the pycnocline, interspersed with sporadic elevated values of up to $10^{-3} \mathrm{~m}^{2} \mathrm{~s}^{-1}$ in the upper $50 \mathrm{~m}$ (Shroyer et al., 2016b; Lucas et al., 2016). Thus the average effective lateral diffusivity is estimated to be $O(0.1) \mathrm{m}^{2} \mathrm{~s}^{-1}$ below $50 \mathrm{~m}$ depth, similar to other estimates, yet an order of magnitude smaller than what was estimated from dye-release experiments in the coastal ocean south of Martha's Vineyard (Sundermeyer and Ledwell, 2001) and in the Sargasso Sea (Shcherbina et al., 2015). However, within the top of the shallow pycnocline, right below the ML, vertical diffusivities of $K_{v}=10^{-5} \mathrm{~m}^{2} \mathrm{~s}^{-1}$ do not seem unreasonable, and thus lateral diffusivities $K_{h}$ could be enhanced to values of $O(1) \mathrm{m}^{2} \mathrm{~s}^{-1}$. In addition, if enhanced vertical shear is correlated with intermittent enhanced turbulent mixing (Kunze and Sundermeyer, 2015), effective lateral mixing could intermittently be an order of magnitude larger.

If shear dispersion in the $\mathrm{BoB}$ is driven by near inertial waves, which generate oscillatory shear, the spatial scales over which the effective diffusivity acts is equal to the spatial scales

of the oscillation's lateral excursions. This scale is estimated as $\Delta z \cdot V_{z} \cdot \frac{1}{f}$, where $\Delta z$ is the vertical scale of the oscillation. Given a shear of $0.01 \mathrm{~s}^{-1}$ across $10 \mathrm{~m}$ thick layers, the horizontal scale is $O(1) \mathrm{km}$. It is thus plausible that on the shallowest isopycnal surfaces in the top $100 \mathrm{~m}$ of the $\mathrm{BoB}$, shear dispersion, acting on lateral gradients of $O(1) \mathrm{km}$ scales, enhances isopycnal diffusivity to $O(1-10) \mathrm{m}^{2} \mathrm{~s}^{-1}$. This would effectively reduce tracer variance at submesoscales, and potentially lead to the steep spectral fall-off, perhaps aided by the low-latitude and high-stratification of the BoB that favor strong NIW shear layers with very thin vertical extent.

\section{Inhibited cascade of coherent water mass anomalies}

A reduction in the downscale cascade of tracer variance from larger scales could also explain the steepened spectral slope of spice variance below $10 \mathrm{~km}$ scales. One way for this to occur is if the tracer distribution is correlated to the flow field in a manner that the eddy stirring 
and straining is ineffective at breaking apart the tracer's $10-100 \mathrm{~km}$ scale patterns. For example, if the tracer distribution is modulated by coherent structures in the flow, or correlated with potential vorticity (PV) anomalies formed by subduction or entrainment of water masses from the surface or frictional bottom (lateral) boundary layers, then the assumption that macro-turbulence is stochastic and homogeneous is invalid. Both in 2013 and 2015, we encountered mesoscale eddies with an anomalous fresh-cold water mass visible in the core Fig.2-14, and reported in (Gordon et al., 2017), while on a smaller scale, submesoscale subduction was seen to create anomalous stratification and water mass anomalies at fronts (Ramachandran et al., 2018). If the observed steep spectral slope is indeed due to an inhibited spice variance cascade, then water mass distributions in the BoB are not a measure of a random passive tracer being isopycnally stirred, but rather a tracer that, though strictly passive, is correlated with dynamical properties and coherent flow structures. However, even if currents are partially correlated with water mass anomalies, the propagating mesoscale eddy flow field would still be expected to encounter uncorrelated tracer gradients of $O(100) \mathrm{km}$ scales, and strain these to $O(1-10) \mathrm{km}$ scales, thereby replenishing the "missing" variance levels along isopycnals in the interior.

\subsection{Concluding Remarks}

We present a synthesis of along-isopycnal spice variance measured from 3 cruises in the Bay of Bengal and find that along isopycnals in the strongly stratified upper $75 \mathrm{~m}$, the tracer variance spectra are much more steeply sloping $\left(\mathcal{P}(k) \sim k^{-3}\right)$ in the submesoscale regime than can be explained by existing theories of 2-dimensional macro-turbulence. The lack of spice variance at 1-10 $\mathrm{km}$ scales relative to the variance levels at 10-100 km scales means that the horizontal gradients between warm-salty and cold-fresh features are too smooth between 1-10 km scales to be consistent with QG or frontogenetic dynamics. This indicates that in this regime, either horizontal mixing is more effective than expected, or water mass anomalies are less effectively stirred and strained into $1 \mathrm{~km}$ scale features. The first, more likely, explanation invokes the importance of submesoscale processes for horizontal mixing, whether they be vortical or wavelike in nature. Enhanced shear dispersion by NIWs with very small vertical wavelengths are a promising candidate. The latter explanation would mean that spice spectral distributions do not reflect stirring by homogeneous macro- 
turbulence of an independent, stochastically distributed tracer, but rather the distribution of a dynamically relevant property.

At depths between 100 and $200 \mathrm{~m}$, the flattening of spectra suggests that tracer stirring dominated by frontogenesis $\left(\mathcal{P}(k) \sim k^{-2}\right)$ transitions towards stirring driven by stratified turbulence or SQG dynamics $\left(\mathcal{P}(k) \sim k^{-5 / 3}\right)$ with depth. These findings differ from similar observational studies in the Atlantic and Pacific Oceans, which did not observe the steep tracer spectra in the submesoscale range, nor the significant flattening of spectra with increasing isopycnal depth. The results attest to the strong stratification and separation between processes stirring the upper $40-70 \mathrm{~m}$ of the pycnocline, the intermediate layers around $100 \mathrm{~m}$, and the more quiescent interior around $200 \mathrm{~m}$.

The results from this study demonstrate that 2-dimensional macro-turbulence in the ocean is not yet completely understood. The lack of $1-\mathrm{km}$ scale variance calls attention to the potential role of submesoscale dynamics and near-inertial waves, or their interactions, in controlling horizontal mixing in the upper ocean, and to potential misinterpretations of spice anomalies. A proper accounting of submesoscale and wave-like oscillatory processes is important for the development of model sub-grid parameterizations. 


\subsection{Supplementary Figures}

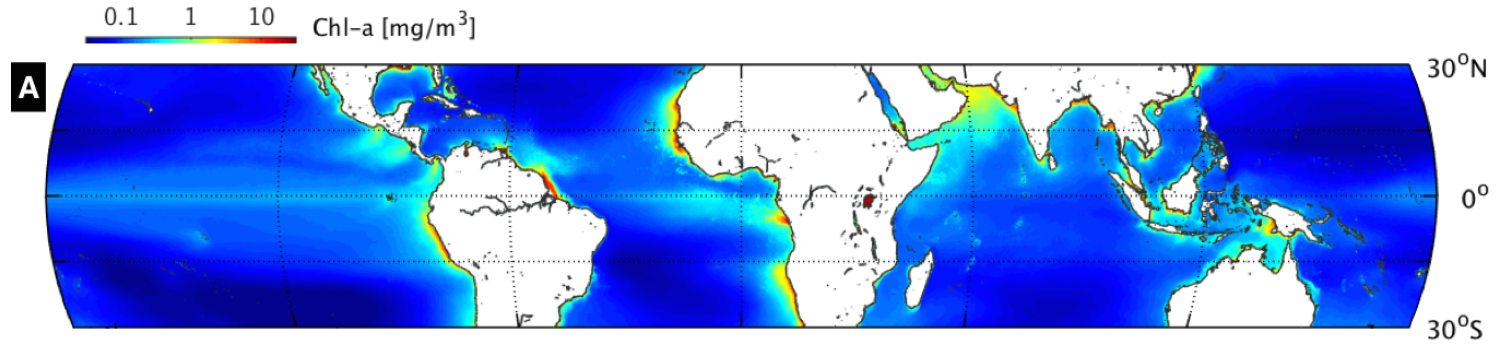

$0 \quad 50 \quad 100$ minimum $\% \mathrm{O}_{2}$ saturation, $0-100 \mathrm{~m}$
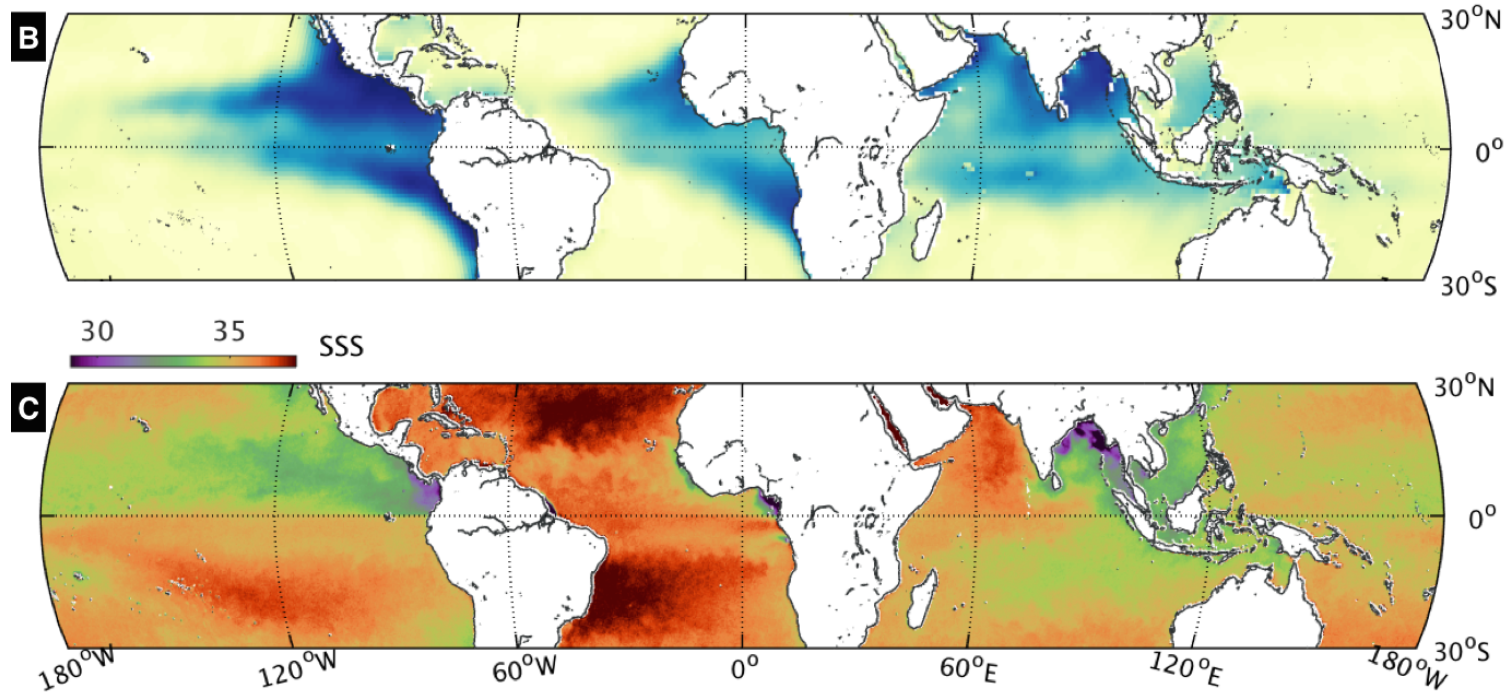

Figure 2-13: Even though the Bay of Bengal's surface biological productivity is not very high, it still has a pronounced oxygen minimum zone (OMZ) below about $75 \mathrm{~m}$ depth. (A) Climatological surface chlorophyl-a concentration, a proxy for biological primary productivity. Compared to other OMZ regions underneath highly productive surface waters, such as the Arabian Sea or the Pacific and Atlantic coastal upwelling regions, the BoB is not very productive. (B) Climatological oxygen saturation at $100 \mathrm{~m}$ depth from WOCE. (C) Surface salinity in winter 2017, measured by SMAP. The low oxygen and low productivity in the $\mathrm{BoB}$ indicate a long residence time and lack of ventilation of subsurface waters insulated by strong freshwater stratification. 


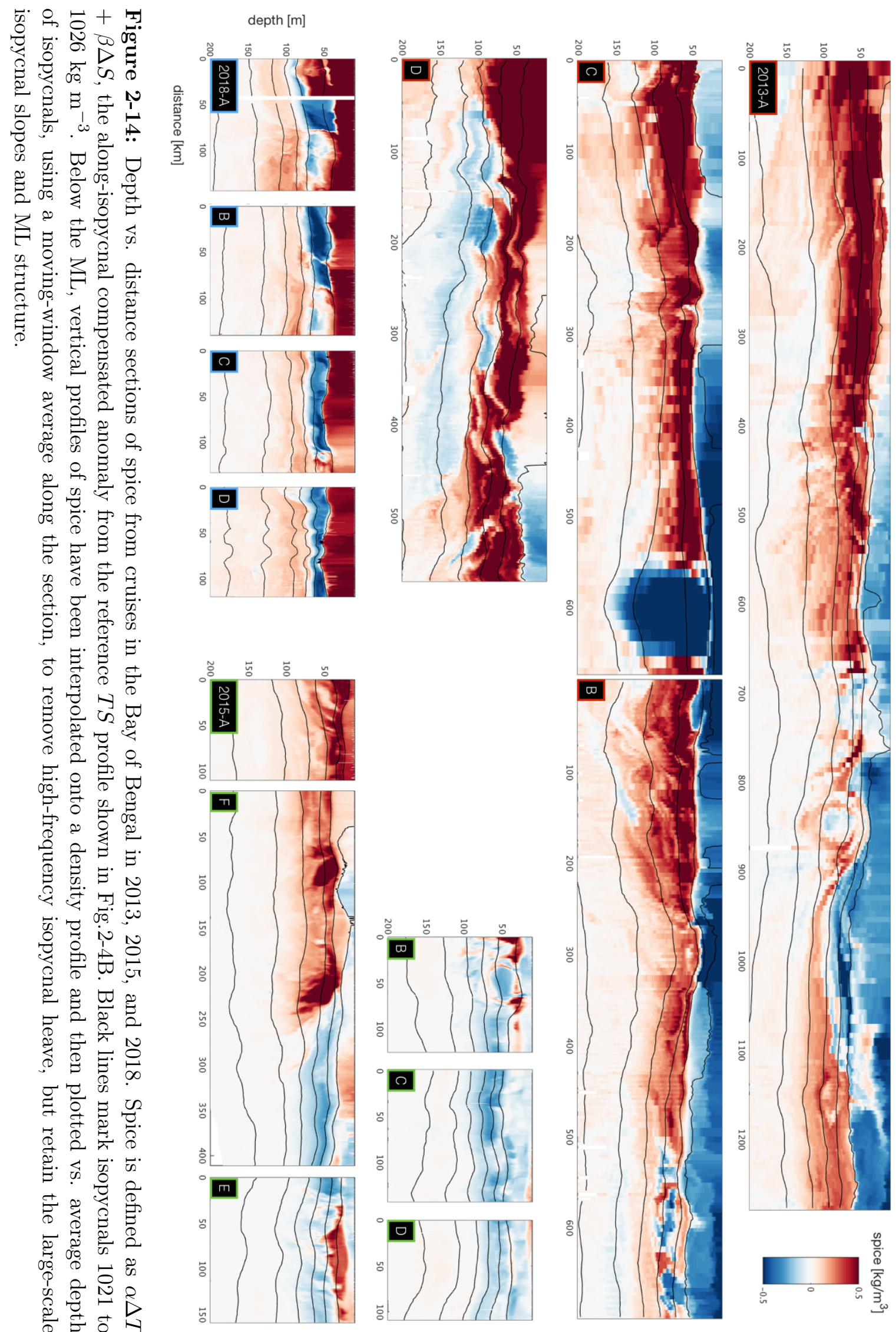



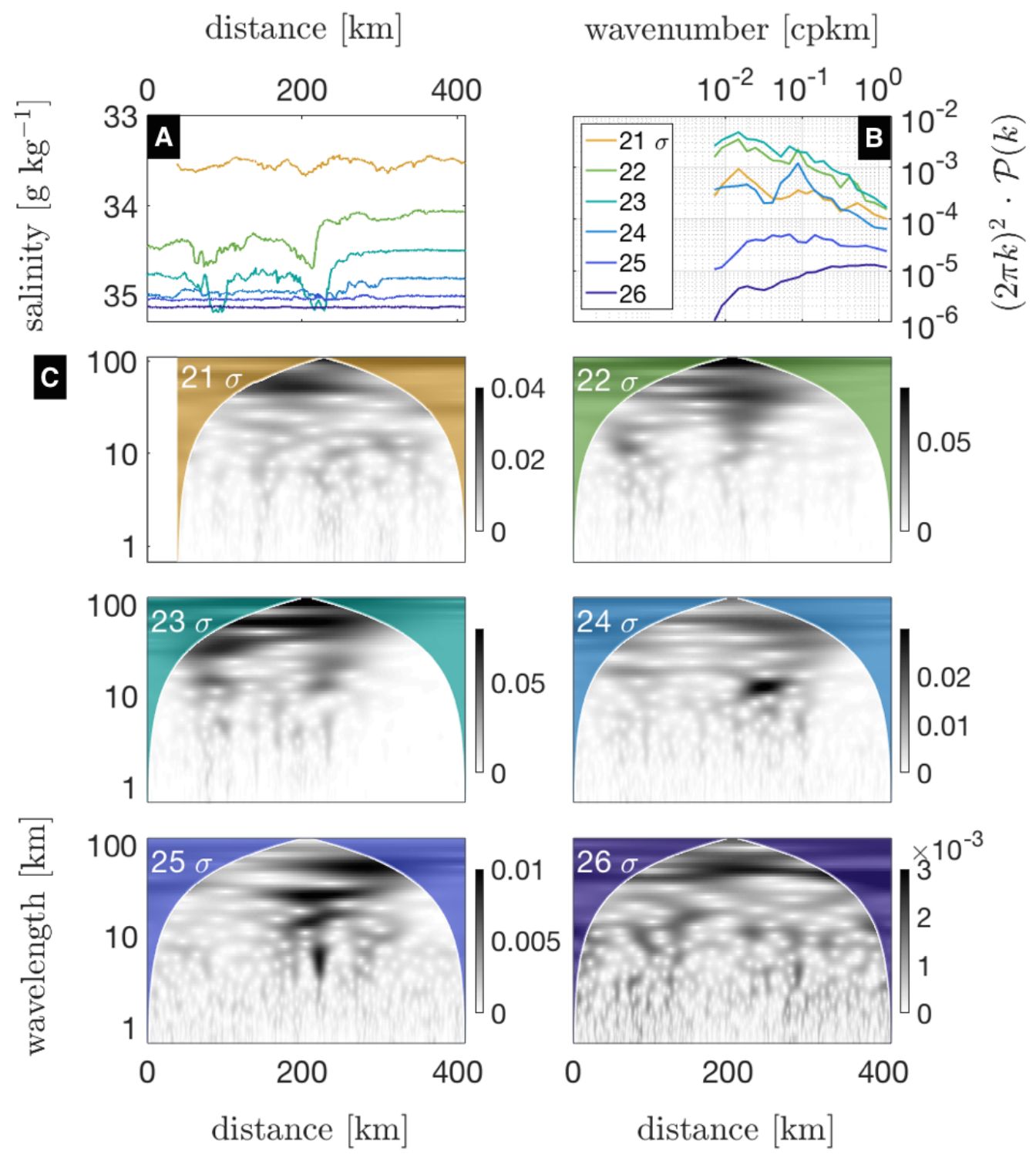

Figure 2-15: Comparison of a wavelet decomposition of isopycnal salinity with the spectral analysis. (A) Salinity in section F from 2015, along $\sigma=21$ to 26 (yellow to blue) isopycnals. (B) Spectral variance decomposition. (C) Wavelet scalogram amplitudes for the different isopycnal salinity records as labeled. Note the change in amplitude ranges is equivalent to the change in spectral levels. Similarly, the increasing relative magnitude of variance at small scales with increasing density is equivalent to the changes in spectral slopes. 
THIS PAGE INTENTIONALLY LEFT BLANK 


\section{Chapter 3}

\section{Formation of Interleaving Layers in the Bay of Bengal}

\subsection{Abstract}

Observations of the upper $200 \mathrm{~m}$ of the strongly stratified Bay of Bengal reveal a host of interleaving layers of water mass properties. The features appear as isopycnal spice anomalies and form multiple, parallel, $\sim 10 \mathrm{~m}$ thin layers, that are coherent over horizontal scales of $30-80 \mathrm{~km}$. They are coincident with anomalies in density stratification and in some cases, with the vertical shear of horizontal velocity, but are not aligned with isopycnals. The cross-isopycnal slopes of these prominent features are $O\left(10^{-4}\right)$ and are, on average, 5 to 10 times smaller than the expected slopes of passive tracer anomalies due to mesoscale stirring. We present two alternate mechanisms by which such interleaving layers can be created from existing lateral spice gradients: (i) stirring by vertically sheared submesoscale eddies, and (ii) near-inertial wave shear. A numerical simulation of a density (and spice) front, based on observations of a narrow and fast jet, develops a submesoscale vortex that produces spice layering similar to our observations. The layering suggests that even a weak vertical diffusivity would greatly enhance the horizontal mixing of spice at submesoscales, the scales of the proposed processes, through shear dispersion. The analysis of the observations draws attention to the prevalence in the Bay of Bengal of submesoscale dynamics and NIWs with unusually high vertical wavenumbers. 


\subsection{Introduction}

Observations of thermohaline patterns such as intrusions, interleaving, or layered water masses, have a useful history of aiding our understanding of ocean dynamics (Schmitt et al., 1987; Richards and Edwards, 2003; d'Orgeville et al., 2004). Ocean stirring, besides shaping patterns of thermohaline anomalies, also affects the distribution of potential enstrophy (potential vorticity variance), as well as chemical and biological particles in the water (Garrett, 1979; Toole and McDougall, 2001). Understanding watermass stirring is thus of both fundamental interest to the study of ocean dynamics, and of practical interest for the prediction of dispersion within the ocean.

The relevance of thermohaline patterns to biological oceanography is further underscored by frequent observations of similar phytoplankton patterns of layering or intrusions (Durham and Stocker, 2012). Thin layers of anomalous fluorescence have been interpreted in different ways, with either biological or dynamical source mechanisms. In such studies, correct identification of source mechanisms is important to correctly understand plankton behaviour and ecology (Lévy et al., 2018). For example, thin phytoplankton layers can indicate either enhanced or suppressed biological activity, controlled by vertical transport of nutrients, cell motility, ageostrophic circulations at fronts, or without any vertical movement by simple horizontal straining and shearing of existing horizontal anomalies (Genin et al., 2005; Durham et al., 2009; Birch et al., 2008; de Verneil et al., 2019).

An advantage of studying thermohaline patterns alongside (or instead of) phytoplankton layering lies in the conserved nature of thermohaline anomalies (Flament, 2002). Since these values are set only by boundary layer temperature and salinity fluxes, and subsequently conserved in the interior until molecularly mixed, their anomalies act as simple markers of the water parcels' origins and movements through the ocean interior. And unlike buoyancy or density, spice, or density-compensated variations in temperature $(T)$ and salinity $(S)$, usually does not directly affect the dynamics (except in the case of double diffusion), and instead act as a passive tracer affected only by the flow dynamics.

This study presents observations of thermohaline features in the Bay of Bengal that are distinguished by multiple, thin horizontal layers of different water masses stacked one above another, in contrast to individual thermohaline intrusions (Shcherbina et al., 2009; Cole and Rudnick, 2012). Understanding the processes that create this layering is important, because 
it reveals what type of flow dynamics dominate horizontal stirring and water mass exchange across the basin, and thus potentially have an effect on vertical density stratification. The observed thinness of the layering has different implications, depending on the timescale of the production mechanism, for mixing and stirring in the BoB.

Some of the observed thermohaline features in the BoB are unlikely to have been created by the traditionally inferred dynamics of either mesoscale currents straining and shearing (Smith and Ferrari, 2009), or double diffusive fluxes driving frontal instabilities or thermohaline staircases (Schmitt, 1994). Instead, submesoscale frontal instabilities, and near inertial internal waves with high-vertical-wavenumber, are identified as the likely dynamical source of these observed layering patterns. We present two simple mechanism by which these processes can create thermohaline patterns with parallel, thin, and long layers.

\subsection{Observations}

\subsubsection{Data Collection}

Hydrographic profiling measurements of the upper ocean were made in the Bay of Bengal (Fig.3-1) between August 24th and September 20th, 2015, aboard the $R / V$ Revelle, as part of the Office of Naval Research Air Sea Interaction Research Initiative (ASIRI). The hydrographic survey was conducted with a Seabird conductivity, temperature, and pressure probe (SBE 49 CTD) mounted in a 'Fast'-CTD system (FCTD) (Pinkel et al., 2012), producing vertical temperature, salinity, and pressure profiles of the upper $200 \mathrm{~m}$ every $~ 3$ minutes while the ship was underway at about $4 \mathrm{kn}$, producing transects with individual profiles spaced about $300 \mathrm{~m}$ in the horizontal. The profiler sampled at $16 \mathrm{~Hz}$, which at free-fall at speeds of about $5 \mathrm{~m} \mathrm{~s}^{-1}$ recorded between 3 to 4 samples per meter in the vertical, resolving vertical gradients at scales of $1 \mathrm{~m}$. The raw conductivity and temperature profiles were quality-controlled, and combined with a small temporal offset to maximize high-frequency lag-correlation (the variable lag, on average about one data point in length, reduced salinity spiking and associated stratification variance). Temperature and salinity were then smoothed with a 5-point median filter, and vertically binned on a regular depth grid. Further details on the FCTD instrumentation and data processing methods are described Chapter 2. The anomalies discussed in this paper are well resolved by the FCTD profiling, greater in magnitude $\left(\gtrsim 0.1 \mathrm{~kg} \mathrm{~m}^{-3}\right)$ over a larger vertical extent $(\gtrsim 10 \mathrm{~m})$ than 
the noise associated with salinity spiking.

Current velocity profiles of the upper $200 \mathrm{~m}$ were made by several hull-, well-, and side-mounted sonar transducers. This study presents currents and shears measured by a $140 \mathrm{kHz}$ transducer system permanently installed on the $R / V$ Revelle, the Hydrographic Doppler Sonar System (HDSS) (Pinkel et al., 2003), configured to sample with $3 \mathrm{~m}$ vertical bins from $20 \mathrm{~m}$ to $\sim 200 \mathrm{~m}$ depth. Additionally, a $300 \mathrm{kHz}$ RD Instruments acoustic Doppler current profiler, temporarily installed at the bottom of a vertical pole attached to the side of the ship's hull, sampled from 10 to $50 \mathrm{~m}$ depth with $1 \mathrm{~m}$ vertical bins. The raw current profile measurements, recording velocities and shears perpendicular and parallel to the ship's direction, were quality controlled, processed into 1 min averages and rotated to eastward and northward velocity components, as well as across-track $U$ and along-track $V$ components. The independent measurements from the different sonar systems matched well in the overlapping depth ranges.

\subsubsection{Data Processing}

For the following analysis, temperature $(T)$, salinity $(S)$, and pressure $(P)$ profiles were vertically interpolated onto a density grid, both to calculate isopycnal averages and anomalies, as well as to graphically present distance-depth sections without the visual contamination of highly variable vertical displacements of neighbouring profiles, caused by internal waves. The density-interpolated profiles were then vertically mapped to a coordinate equal to the section-mean density depth profile, thus preserving the average vertical isopycnal spacing while still presenting isopycnals as flat horizontal lines and removing isopycnal heave (Fig.32).

Since vertical profiles of horizontal current velocity were measured from ship-board transducers, and not in-situ by a lowered instrument with coincident $T S$ measurements, horizontal velocity cannot be directly interpolated onto the same isopycnal grid as $T$ and $S$. Instead, density profiles measured by the FCTD profiler, horizontally more widely spaced but vertically more finely resolved than the HDSS profiles, were horizontally and vertically interpolated onto the velocity profiles. Even though the interpolation imperfectly matches density to velocity bins, and thus unresolved vertical heave still affects the current profiles (Fig.3-2B), the isopycnally adjusted current and shear sections can be well compared with the isopycnal $T S$ sections and derived quantities. 


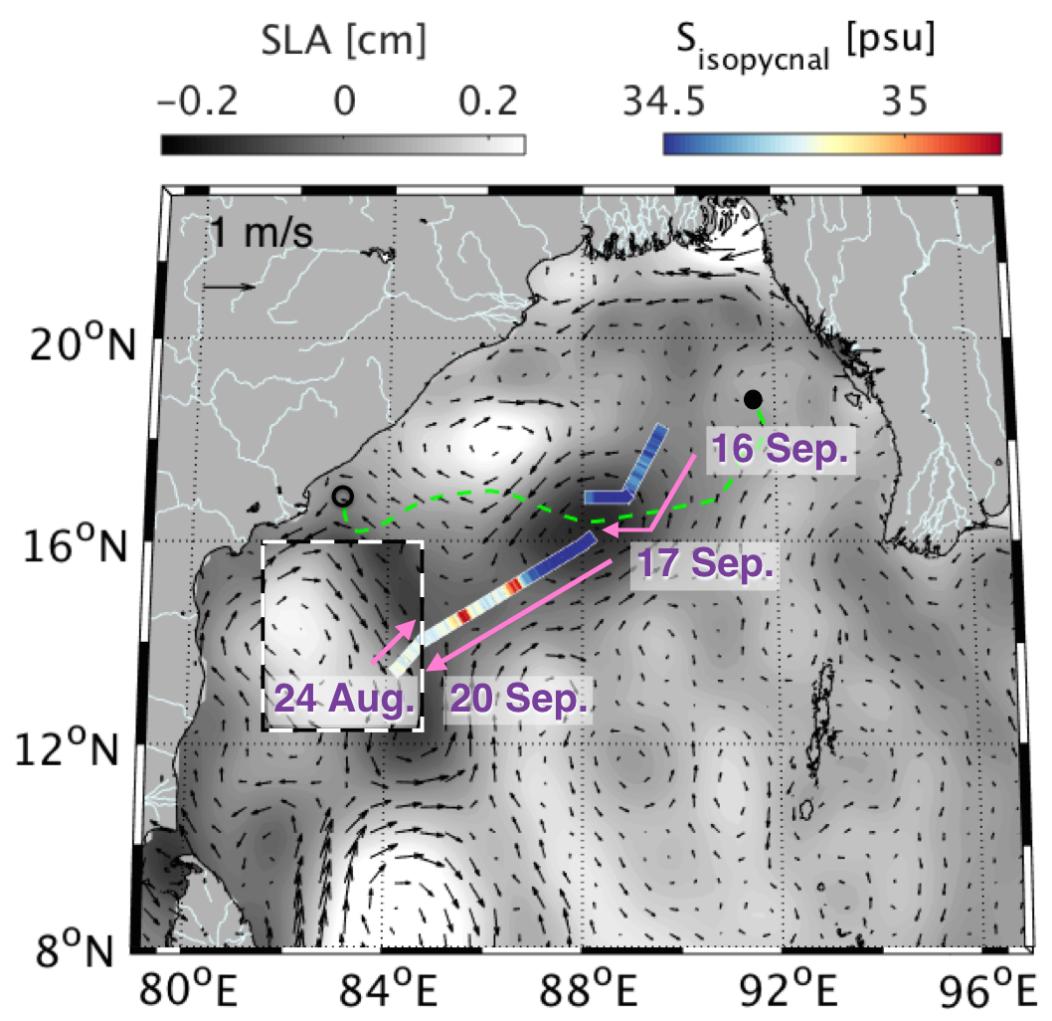

Figure 3-1: Map of the sea level anomaly (gray shades) and associated geostrophic surface currents (arrows) in the Bay of Bengal on September 17, 2015 (inset: August 24), approximately coincident with the ship transects, along which we show salinity at the $23 \sigma$ isopycnal surface (blue to red color, pink arrows indicate transect direction). The ship sections through the large cyclonic eddy (negative SLA) revealed anomalously fresh-cold water (marked blue) in the upper $100 \mathrm{~m}$ of its core, compared to water outside the eddy. The dashed green line shows the track of the center of this eddy over 10 months. It formed around April 1, 2015 in the east (filled black circle), traveled westward, and merged into the East India Coastal Current around February 1, 2016 (open black circle).

Thermohaline water mass contrasts are revealed by calculating isopycnal spice (Fig.32C). Spice is defined along an isopycnal as $\alpha d T+\beta d S$, where $d T$ and $d S$ are equal to the difference in $T$ and $S$ from a reference watermass $T S$ value, and $\alpha$ and $\beta$ are the thermal expansion and haline contraction coefficients, respectively (Flament, 2002). The reference $T S$ profile is created from the density-gridded sections of $T$ and $S$, defined as the smoothed profile of median $T$ and $S$ vs. density. Spice reveals anomalous thermohaline below the surface mixed layer (Fig.3-2C), such as the fresh and cold (minty) watermass lens in the core of the cyclonic eddy, in between 1021 and $1024 \mathrm{~kg} / \mathrm{m}^{3}$ isopycnals, otherwise obscured by the large vertical gradient in $S$ and $T$. South of the eddy, two salty and warm (spicy) patches stand out. Closer inspection of the spice patterns reveals smaller-scale horizontal 
variance too, such as north of the cyclonic minty eddy, or towards the southwestern end of the survey, where spice is vertically layered.

Such vertical changes in water mass are identified by diapycnal spiciness curvature (DSC)

(Fig.3-2D), defined by Shcherbina et al. (2009) as $\sigma_{z}^{-1} \frac{d}{d z}\left(\frac{\alpha T_{z}+\beta S_{z}}{-\alpha T_{z}+\beta S_{z}}\right)$. Under typical ocean conditions where $\alpha_{z} T_{z}<<\alpha T_{z z}$ and $\beta_{z} S_{z}<<\beta S_{z z}$, DSC can be well approximated as $\approx 2 \alpha \rho T_{z z} \approx 2 \beta \rho S_{z z}$. DSC anomalies mark peaks in curvature in TS space, effectively highlighting the local maxima and minima in isopycnal spice along a vertical profile. It is equivalent to, and can be conveniently calculated as, the vertical gradient in density space of the tangent of the Turner angle, the latter being a vertical gradient quantity ( $\equiv$ $\left.\tan ^{-1}\left(\alpha T_{z}+\beta S_{z}\right) /\left(\alpha T_{z}-\beta S_{z}\right)\right)$, readily computed by numerical seawater processing and analysis tool-kits. Besides being easily computed, DSC has several other benefits. Unlike spice, DSC is independent of any definition of a reference $T S$ profile. It is also independent of vertical strain or density stratification, only sensitive to thermohaline water mass layering. It is a useful metric to identify vertical changes in watermass characteristics.

\subsubsection{Hydrography and Mesoscale Setting}

The upper $200 \mathrm{~m}$ of the ocean were characterised by a relatively fresh $\left(31-33 \mathrm{~g} \mathrm{~kg}^{-1}\right)$ and warm $\left(\sim 30^{\circ} \mathrm{C}\right)$ surface mixed layer $(\mathrm{ML})$ between $10 \mathrm{~m}$ and $30 \mathrm{~m}$ deep, beneath which a sharp halocline (salinity increasing to $35 \mathrm{~g} \mathrm{~kg}^{-1}$ ) and more gradual thermocline form a strongly stratified upper ocean. Density ranged from $1019 \mathrm{~kg} \mathrm{~m}^{-3}(\sigma \equiv 19)$ in the freshest surface layers, to $1026.5 \mathrm{~kg} \mathrm{~m}^{3}(\sigma \equiv 26.5)$ around $200 \mathrm{~m}$ depth. Beneath the ML, density stratification $N^{2}\left(\equiv-\frac{g}{\rho_{o}} \frac{\partial \rho}{\partial z}\right.$ ) was strong in the upper $50 \mathrm{~m}$ (peak $N^{2} \sim 1.5 \times 10^{-3} \mathrm{~s}^{-2}$ ), gradually weakening with increasing depth $\left(N^{2} \sim 1 \times 10^{-4} \mathrm{~s}^{-2}\right.$ at $\left.200 \mathrm{~m}\right)$. Equivalently, buoyancy frequencies $N\left(\equiv \sqrt{N^{2}}\right)$ ranged from about $0.04 \mathrm{~s}^{-1}$ to $0.01 \mathrm{~s}^{-1}$, or oscillation periods $(\equiv 2 \pi / N)$ of about 2 to 10 minutes. The average vertical gradients of both temperature and salinity were individually stable, with only occasional small-scale inversions, such as beneath two $\sim 50 \mathrm{~km}$ long patches of salinity maxima at $50 \mathrm{~m}$ depth that were crossed in the longest section. Vertical shear was dominated by high-wavenumber shear layers, with an average root mean square magnitude of $0.01 \mathrm{~s}^{-1}$ in the upper $200 \mathrm{~m}$ (vector direction of vertical shear shown in Fig.3-2B).

The cruise encountered two major mesoscale features visible in sea level anomaly (SLA) maps in Fig3-1. At the beginning of the cruise, the ship crossed a strong frontal jet directed 

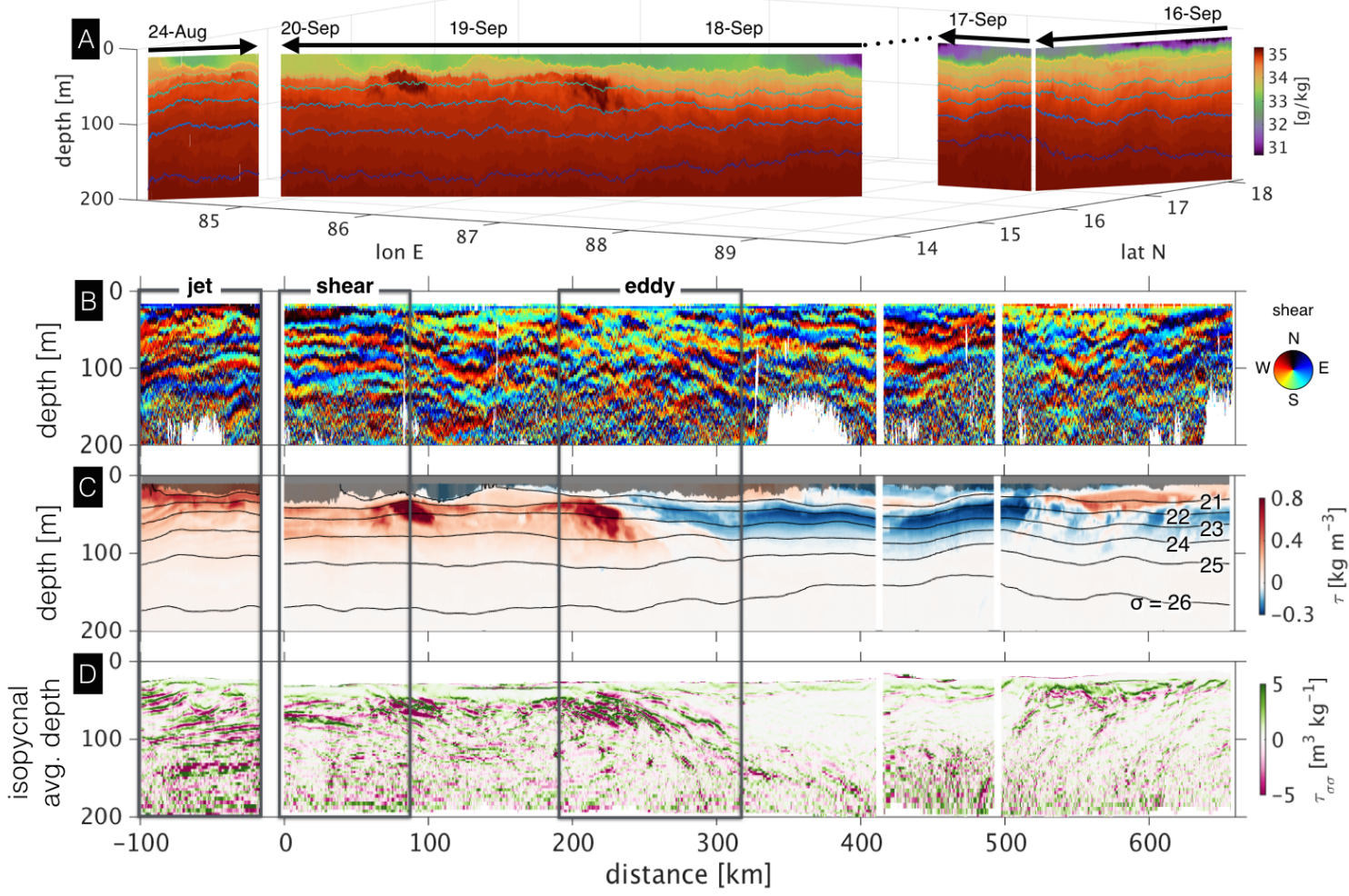

Figure 3-2: Lateral view of the sections mapped in Fig.3-1. (A) Salinity in the upper $200 \mathrm{~m}$, plotted vs. latitude and longitude. The arrows and dates at the top of the sections mark the ship's passage. Thin lines mark isopycnals 21 to $26 \sigma$. Black arrows mark the location of the $T S$ profiles shown in Fig.3-5. (B) Cardinal direction of the vertical shear, measured by the $R / V$ Revelle's $140 \mathrm{kHz}$ HDSS with $3 \mathrm{~m}$ vertical bins in the upper $200 \mathrm{~m}$. The 4 sections, horizontally mapped to along-section distance, are shown next to each other, note though that the relative position has been adjusted for clarity of presentation. Boxes mark the regions shown in detail in Fig.3-3. (C) Spice $(\tau)$, defined as the along-isopycnal watermass anomaly from a reference (defined as a smoothed average) TS profile. The individual spice profiles along the section have been vertically mapped to isopycnal depth, locally averaged over $\sim 10 \mathrm{~km}$, to remove the high-frequency vertical heave and strain, but retain the average sloping features of the isopycnals. Black lines mark isopycnals, gray shading marks the surface mixed layer depth, defined by a $0.1 \sigma$ increase in density from the surface. (D) Diapycnal Spiciness Curvature (DSC or $\tau_{\sigma \sigma}$ ), vertically mapped to isopycnal depth averaged within each section, thus isopycnals are flat.

southeastward and offshore between a positive and negative SLA (map inset), the jet was crossed three times with circa $100 \mathrm{~km}$ long repeat sections. Towards the end of the cruise, the ship transected a $\sim 300 \mathrm{~km}$ wide eddy cyclonically rotating around a $\sim 17 \mathrm{~cm}$ strong SLA depression, 3 successive sections cumulatively span about $650 \mathrm{~km}$.

The mesoscale eddy, dominating surface currents and SLA, additionally has an imprint in the hydrography below the surface mixed layer. The sections through the eddy reveal 
an anomalously cold and fresh ('minty') watermass in the upper $100 \mathrm{~m}$ inside of the eddy (Fig.3-2C). Furthermore, the minty interior has reduced DSC magnitudes and variance in contrast to the rest of the sections (Fig.3-2D). This indicates that the anomalous and uniform water mass in the eddy's core has coherently propagated with the eddy currents into the interior of the BoB. Satellite SLA maps before and after the cruise period reveal the cyclonic eddy had formed as a distinct isolated anomaly around 1st April 2015, near the northeastern corner of the BoB, traveled south-westward, and persisted as a distinct anomaly until merging into a coastal SLA feature around 1st February 2016, completing a 10 months long journey (path marked in Fig.3-1). When the ship crossed the cyclonic eddy, in September 2015 near $88^{\circ} \mathrm{E}$, the eddy had already traveled roughly 5 months and $500 \mathrm{~km}$ from the northeastern edge of the BoB.

In contrast to the lack of watermass layering in the top $100 \mathrm{~m}$ inside the eddy, DSC variance is elevated in several areas at its edges, and outside of it. Three prominent layering features, identified in Fig.3-2, are associated with three prominent features in surface and sub-surface currents. (i) 'Eddy', the edge of the aforementioned mesoscale eddy, in between its cold-fresh interior water mass and a warm-salty watermass patch to the southwest of it. (ii) 'Jet', the strong narrow current at the beginning of the cruise. (iii) 'Shear', the final (southwestern) segment of the long section, which, instead of prominent currents, is marked by prominent shear layers. These segments are shown in detail in Fig.3-3.

\subsection{Analysis of Layering Patterns}

\subsubsection{Slopes}

The three $\sim 100 \mathrm{~km}$ long section details contain several different types of spice patterns. The 'eddy' segment, dominated by an along-isopycnal watermass gradient, shows many fragmented DSC anomalies, sloping across isopycnals, laterally coherent over at most $\sim 30 \mathrm{~km}$. In contrast, the 'jet' segment contains DSC anomalies that are coherent over its entire $80 \mathrm{~km}$ length, also crossing isopycnals but with a shallower slope. Similarly, the 'shear' segment contains nearly flat DSC layers coherent over at least $50 \mathrm{~km}$, possibly longer if extending beyond the end of the observed section. A measure of the slope distribution of water mass features is shown in Fig.3-4, measured by the joint probability distribution of vertical and horizontal gradients of DSC. The ratio of vertical to horizontal gradient magnitudes is a 


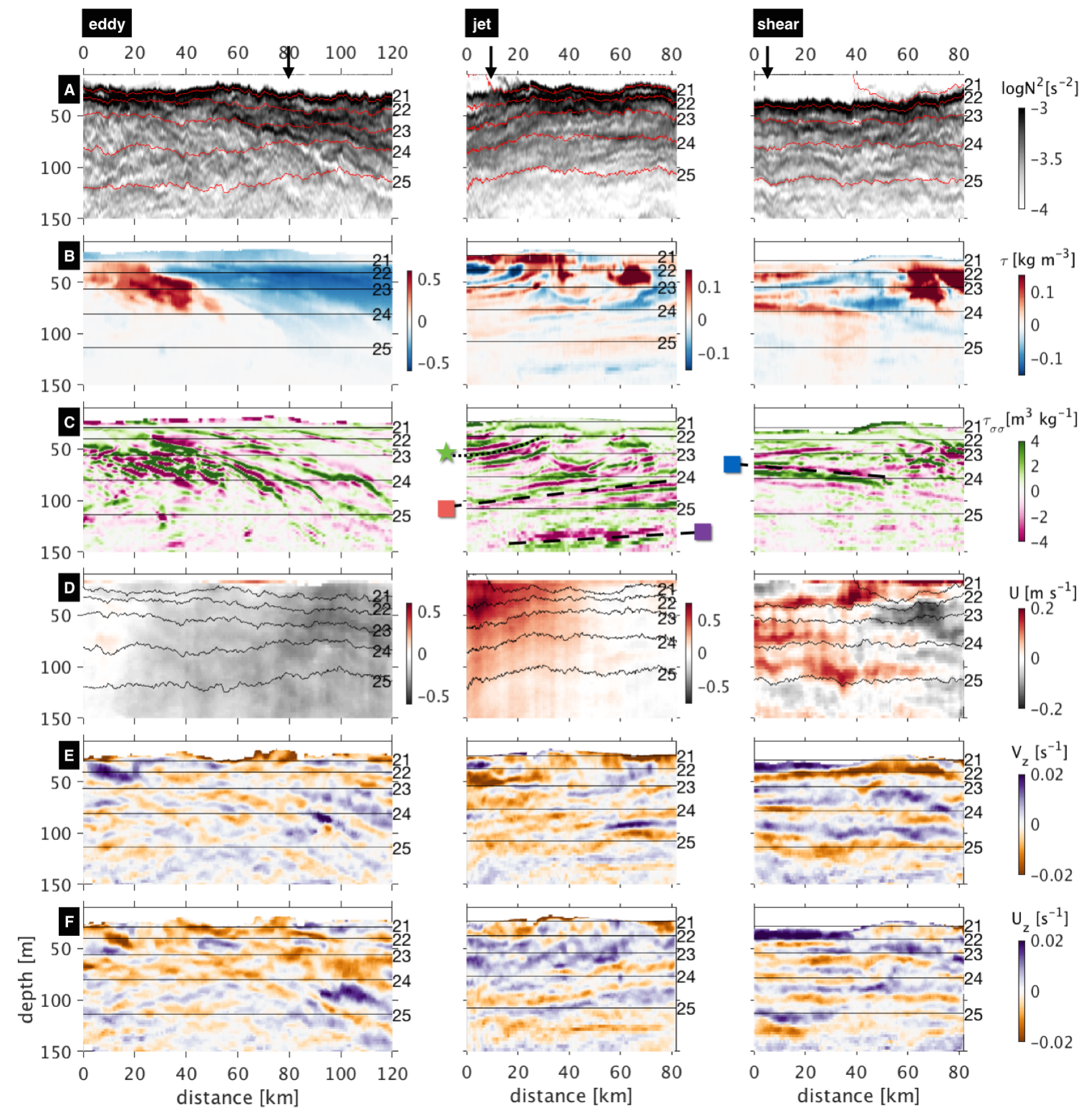

Figure 3-3: Details from the sections shown in Fig.3-2. Eddy, the southern edge of the negative SLA and the cyclonically rotating currents. Jet, crossing a strong $\left(\max \sim 1 \mathrm{~m} \mathrm{~s}^{-1}\right)$ and narrow jet of surface current. Shear, area with weak currents but prominent vertical shear layers. (A) $\log _{10}$ of density stratification $N^{2}$. Red lines mark isopycnals 21 to 25 $\sigma$. (B) Isopycnal Spice $\tau$, plotted vs. the section average isopycnal depth, hence isopycnals (black lines) are flat. (C) Diapycnal Spiciness Curvature $\tau_{\sigma \sigma}$. The slanted dashed lines mark prominent layering, characteristics of which are shown in Fig.3-6. (D) Across-track current velocity (red $=$ out of the page) plotted vs. depth. (E) Vertical shear, acrosstrack component and $(\mathbf{F})$ along-track component, interpolated onto density and plotted vs. section-average isopycnal depth.

measure of slopes, since a steeply sloping anomaly will have a larger magnitude horizontal gradient than a nearly flat anomaly, which will have a small (zero if perfectly flat) horizontal 
gradient, for a given vertical gradient magnitude (Smith and Ferrari, 2009; Cole and Rudnick, 2012). The center of the observed distribution corresponds to a slope of $\sim 5 \times 10^{-4}$, with a majority of the slopes smaller than $1 \times 10^{-3}$. The average slope of only the prominent layers, marked by dashed lines in Fig.3-3, is $\sim 3 \times 10^{-4}$, marked by a white dashed line in Fig.3-4.

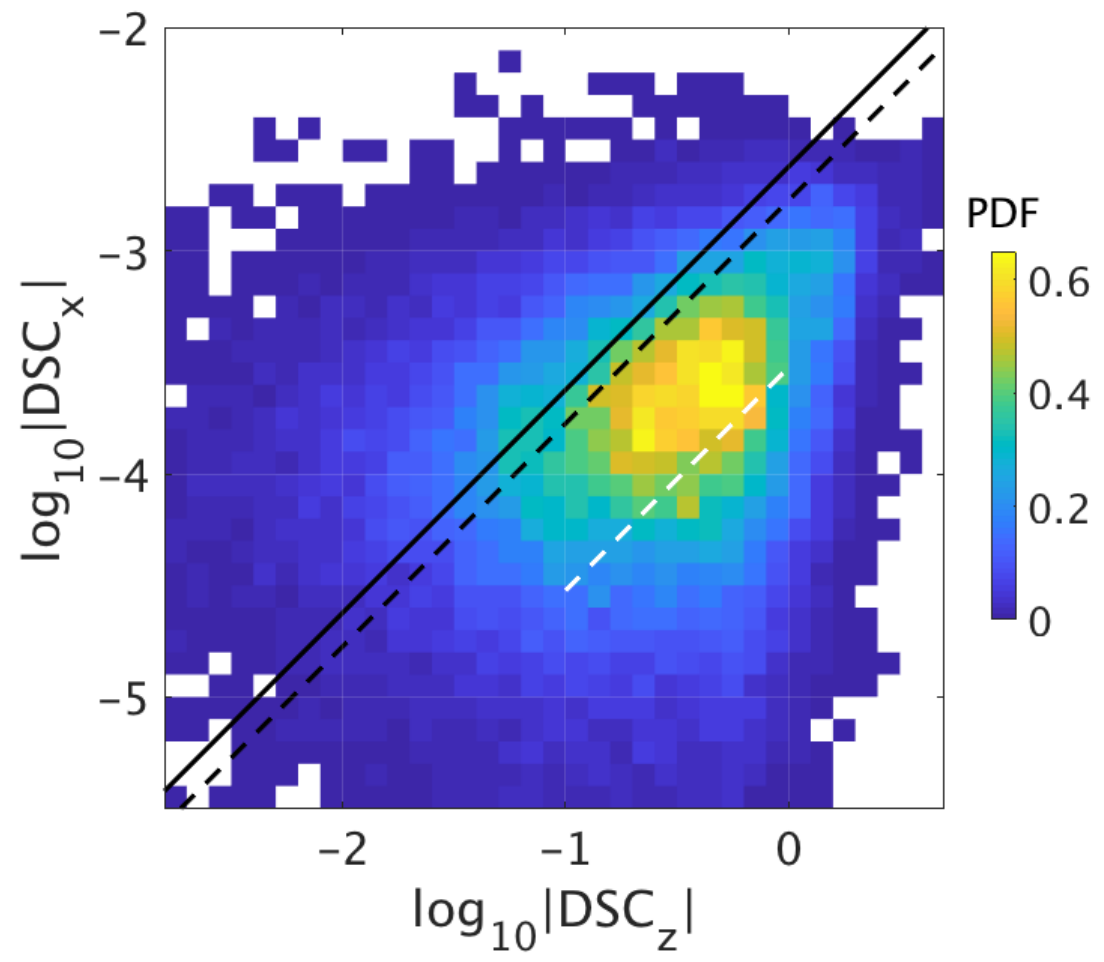

Figure 3-4: Slopes of passive watermass tracer layers in the sections shown in Fig.33, estimated by a 2D PDF of the vertical and horizontal gradients of DSC. For a given vertical gradient, a smaller horizontal gradient signifies a shallower slope. The black solid line corresponds to a slope of the average aspect ratio $f / N \sim 2 \times 10^{-3}$ (dashed line $f / \sqrt{2} N$, ) calculated between $50 \mathrm{~m}$ and $150 \mathrm{~m}$ depth, with $N^{2}=10^{-3.5} \mathrm{~s}^{-2}$, and $f\left(15^{\circ}\right) \sim 4 \times 10^{-5} \mathrm{~s}^{-1}$. The white dashed line indicates the average slope $\left(3 \times 10^{-4}\right)$ of the prominent layering marked by lines in Fig.3-3.

It is worth noting the dependence and sensitivity of this measure of layer slopes on gradients at the shortest horizontal scales. Because of the gradient operator's inherent small scale of analysis, even a spice pattern of long flat layers will produce a slope distribution containing steep features, if the observations contain some measurement noise adding profile to profile horizontal spice changes. Since, here, we are interested in measuring the slope 
distribution of the patterns of long spice layers coherent over $10 \mathrm{~s}$ of $\mathrm{km}$, we reduced the short-scale noise by applying a short-scale 2-dimensional smoothing filter to the DSC sections before computing the slopes. The slope distribution is not very sensitive to the scale or strength of the smoothing, as long as some smoothing is applied to remove variance at the shortest resolved scale. The validity of the slope distribution is confirmed by measuring the actual slopes of the prominent layers, which is close to, and still somewhat smaller than the center of the distribution.

\subsubsection{Coincident Stratification Layering}

In the three featured section details, spice layering is accompanied by parallel sloping layers of anomalous density stratification. The coincident anomalies can be seen in Fig.3-5, plotting several adjacent vertical $T S$ profiles in the upper $100 \mathrm{~m}$, coloured by density stratification $N^{2}$, for one location within each segment marked by black arrows in Fig.3-3. The wiggles in $T S$ space correspond to isopycnal spice or DSC anomalies, which do not necessitate any changes in density gradients, since changes in $T$ can be fully density-compensated by changes in $S$. Similarly, changes in vertical density stratification can occur without any changes in spice, since a water column with a single $T S$ profile can be vertically squeezed or stretched, changing only the vertical density gradient. However, in these three sections, both spice and $N^{2}$ show the same $\sim 10 \mathrm{~m}$ spaced alternating anomalies with depth, with values of $N^{2}$ alternating between a minimum of $\sim 10^{-3} \mathrm{~s}^{-2}$ and a maximum up to $\sim 10^{-3} \mathrm{~s}^{-2}$. Yet the phase relationship between the anomalies are different for each of the three sections. For example, in the top $70 \mathrm{~m}$ of the 'jet' profiles, increased stratification anomalies coincide with the watermass switching from minty to spicy with increasing depth, whereas in the 'shear' profiles, the increased stratification anomaly coincides with minty anomalies.

\subsubsection{Multiple Parallel Layers}

Four instances of multiple parallel spice layers, coherent over $10 \mathrm{~s}$ of $\mathrm{km}$, stand out in the sections of Fig.3-3. Marked by dashed lines in the 'jet' and 'shear' segments, properties of these layers are shown in Fig.3-6. The prominent layering patterns are distinguished by the following characteristics: (i) shallow $O\left(10^{-4}\right)$ cross-isopycnal slopes, (ii) multiple, parallel, $\sim 10 \mathrm{~m}$ thin, layers in spice anomaly, (iii) laterally coherent over at least $30 \mathrm{~km}$ to $80 \mathrm{~km}$ in

length, possibly longer, and (iv) $N^{2}$ (density stratification) anomaly layers coincident with 

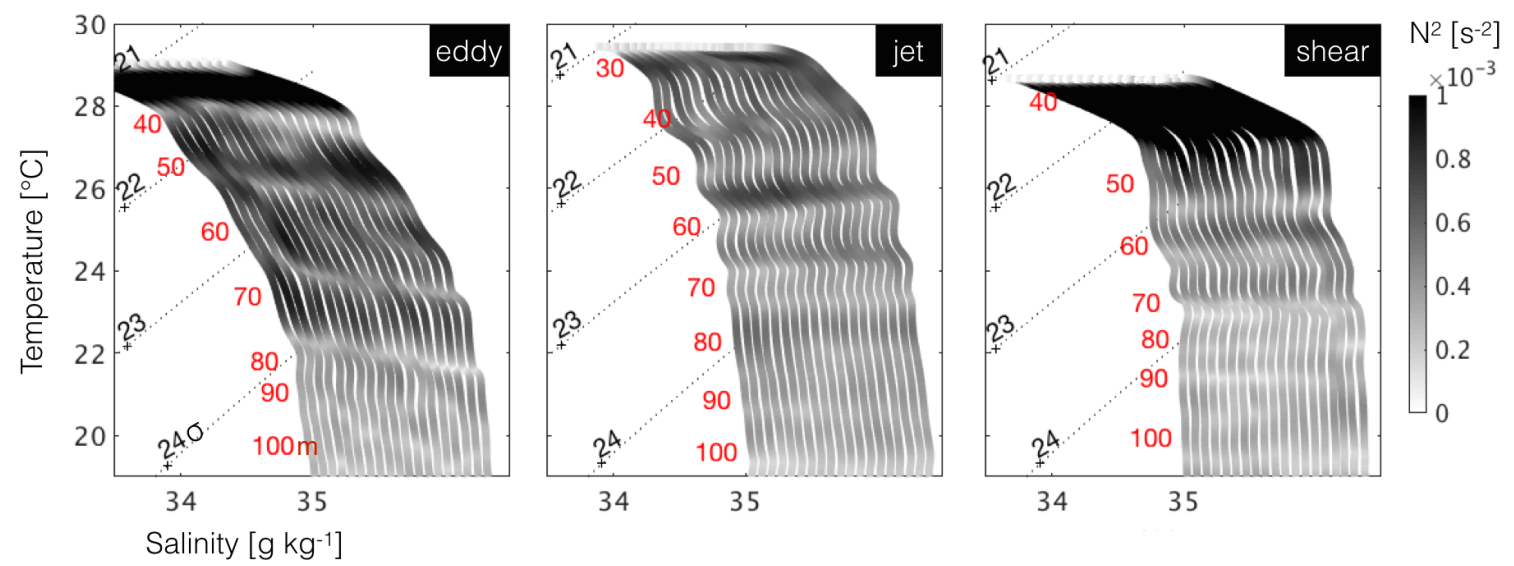

Figure 3-5: TS profiles from the sections shown in Fig.3-3,locations marked by black arrows. Each panel shows 19 consecutive profiles, spanning about 1 hour and $6 \mathrm{~km}$ along the ship track. Gray shading shows density stratification $N^{2}$, revealing that anomalies in both watermass and stratification have similar vertical and lateral coherence. Dotted contours show the density for the first profile, red labels show the depth. Each consecutive profile is shifted slightly to the right (starting from the first profile) to generate this waterfall plot.

the spice (water mass) layers.

\subsection{Mechanisms for Layer Formation}

What produces spice layers in the ocean? Layered water mass anomalies can be produced by mesoscale currents straining and shearing a background gradient in water mass characteristics or spice (MacVean and Woods, 1980). We briefly review the salient aspects of this formation mechanism of tracer layers, before proposing two additional mechanisms capable of creating thin tracer layers. We hypothesize that these mechanisms are active in the Bay of Bengal, co-existing with mesoscale filamentation of tracers, and are potential sources of the observed thin, nearly flat, parallel spice layers.

\subsubsection{Existing Theory: Mesoscale Stirring of Tracer}

Mesoscale horizontal strain and horizontal shear sharpens horizontal gradients, and vertical shear differentially advects, and, thus, tilts these horizontal gradients (and cross-isopycnal features) into long, thin, sloping layers (Haynes and Anglade, 1997). Countering this production, vertical mixing erases spice layers and their vertical water mass variance (Smith and Ferrari, 2009). 


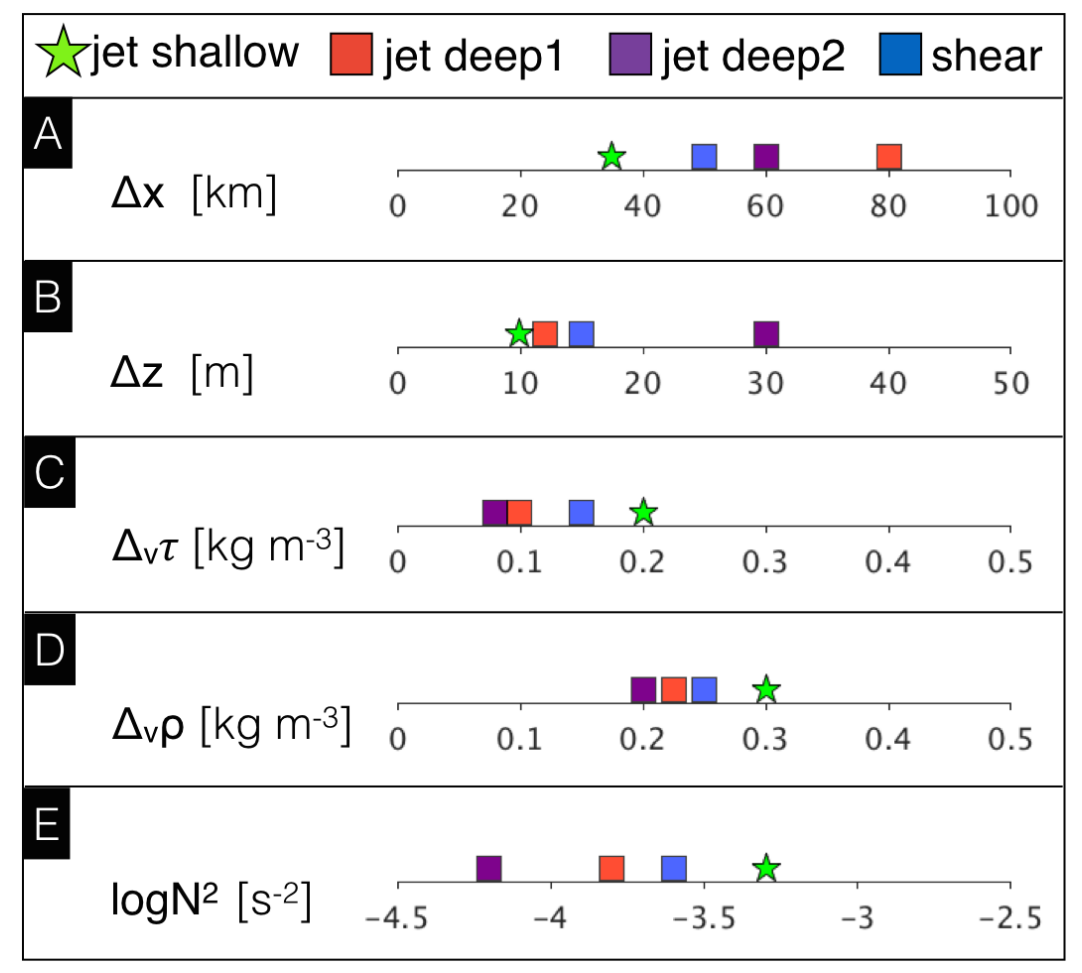

Figure 3-6: Properties of the prominent layering marked in Fig.3-3 by black dashed lines and corresponding symbols in the 'Jet' and 'Shear' sections, values are estimates with about $10 \%$ uncertainty. (A) Horizontal extent, lower limit, since the layering patterns likely extended beyond the end of the ship tracks. (B) Vertical thickness of individual layers within the patterns of multiple parallel layers. (C) Magnitude of the vertical spice anomaly. (D) Vertical change in density across thickness of individual layers. (E) Mean density stratification $N^{2}$ at the depth of the layers.

The large-scale distribution of spice layering in our observations, marked by suppressed DSC variance within the mesoscale eddy's interior, and elevated variance at the eddy's edges, fits into the framework of mesoscale stirring of spice (Meunier et al., 2015). The lack of DSC variance in the eddy's interior is consistent with trapped waters being advected coherently with the eddy's SLA and currents, with no spice layering forming in the 5 months between the eddy's origin in the BoB's northeastern coastal region and our observations. In contrast, spice gradients at the eddy's edges are layered, consistent with strain and shear acting at the eddy's edge. The salinity maximum patch at the eddy's southern edge, is likely a long salty filament advected by the mesoscale flow from the south (Fig.3-1, stretched around by the eddy's rotating currents, and seen in cross-section.

Horizontal strain cascades horizontal tracer gradients to higher wavenumbers (smaller scales), and vertical shear cascades gradients to higher vertical wavenumbers (thinner layers), 
while also elongating the layer extent. The aspect ratio of vertical thickness to horizontal extent keeps decreasing, until the vertical wavenumber cascade is arrested by isotropic turbulence mixing away vertical gradients. If the horizontal strain to vertical shear ratio is increased, for a given vertical shear (and thus tilt) tracer anomalies become more horizontally strained (and thus thinner). Thinner tracer features are mixed away more quickly, which arrests the tilting and decreasing of slopes (flattening). Thus the ratio between horizontal strain and vertical shear determines the average slope of tracer features (Smith and Ferrari, 2009). At small scales in a quasigeostrophic dynamics framework, that ratio is approximately equal to $f / N$, the ratio between the Coriolis parameter (inertial frequency) and vertical stratification (measured by the buoyancy frequency) (Charney, 1971).

The aspect ratio $f / N$ in the stratified interior of the BoB (between $\sim 50 \mathrm{~m}$ and $\sim 150 \mathrm{~m}$ depth) was on average $\sim 2 \times 10^{-3}$ (given $f\left(15^{\circ}\right) \sim 4 \times 10^{-5} \mathrm{~s}^{-1}$, and average $N=0.02$ $\mathrm{s}^{-1}$ ). Alternatively, using the average mesoscale strain, is of order $10^{-5} \mathrm{~s}^{-1}$ (calculated from AVISO currents), and the average observed shear magnitude of $10^{-2} \mathrm{~s}^{-1}$, the strain to shear ratio is also estimated to be of $O\left(10^{-3}\right)$. However, the observed mean tracer slope is four-fold smaller $\left(\sim 5 \times 10^{-4}\right)$, with few tracer slopes observed to be as steep or steeper than the predicted $f / N$ slope (black line in Fig.3-4), and the most prominent layer slopes are almost ten-fold smaller $\left(\sim 3 \times 10^{-4}\right)$. Furthermore, while mesoscale stirring creates layers of passive tracer anomaly, along-isopycnal currents are not expected to create coincident layers in density stratification anomaly, as we observed in the BoB. Lastly, as will be shown in the next section, the mesoscale currents in the 'jet' and 'shear' sections were dominated by submesoscale and internal wave characteristics, strongly altering the shear. While mesoscale stirring can create spice layering, the set of characteristics of the spice layering observed in the $\mathrm{BoB}$ raises the question of what alternative mechanism could create such features.

\subsubsection{New Hypothesized Layering Mechanisms in the Bay of Bengal}

What is the source mechanism that created these prominent, parallel, 10's of km long, very gently sloping layers of watermass and density stratification anomalies? High-wavenumber layering in instantaneous density stratification $N^{2}$, i.e. alternating anomalies of enhanced and diminished vertical gradients of density, is usually interpreted as the effect of vertical strain by internal gravity waves (IGW) (Garrett and Munk, 1979). But the vertical displacements associated with IGWs do not create spice layering. On the other hand, mesoscale 
isopycnal stirring does not create density stratification anomalies. Nonetheless, we observe coincident layering in both, as well as coincident shear layers of the same small vertical scale.

Double diffusive fluxes are thought to be able to create cross-isopycnal layering of spice as well as stratification anomalies (Ruddick and Turner, 1979). However, any double diffusive instability mechanism needs a vertical inversion of either salinity or temperature, or a compensated thermohaline front from which to extract energy (Schmitt, 1994). Yet here, across the upper $200 \mathrm{~m}$, both salinity and temperature are generally stably stratified. The layering in the 'jet' and 'shear' sections is neither within a large-scale $T$ or $S$ inversion nor a thermohaline front, and thus unlikely created by salt fingering or diffusive convection instabilities. This does not rule out that double diffusive fluxes might be active in between the anomalous spice layers, since salinity profiles occasionally contain inversions caused by the layering. Such fluxes would reduce the magnitude of the spice anomalies, while producing alternating anomalies of weakened and strengthened density stratification. However, it is unclear whether double-diffusive instabilities would grow to significance in the highly sheared environment. Instead of double diffusive instabilities, we propose two alternate mechanisms to explain the observations of multiple, thin, flat, coincident layers of watermass and stratification anomaly.

\subsubsection{Hypothesized Mechanism A: Submesoscale Sheared Eddy Twist}

The first proposed mechanism, which produces multiple parallel spice layers with lateral extent of a few 10's of km, involves stirring by a submesoscale eddy across a front of both spice and density. While stirring by any sheared flow, such as mesoscale currents with shear, can produce thin, sloping, spice layers, such currents cannot produce multiple parallel layers, unless initially there were multiple parallel vertical gradients (Franks, 1995). In contrast, a submesoscale eddy, formed at a front, can twist a single horizontal spice gradient around multiple times, creating multiple parallel vertical layers.

The region of the 'jet' section, traversed three times within about 36 hours along a line perpendicular to the mesoscale flow (Fig.3-7A), contained variablity consistent with a submesoscale instability or eddy. Surface-intensified with maximum observed speeds of $1 \mathrm{~m} \mathrm{~s}^{-1}$, the observed jet was not restricted to the ML, and extended into the stratified pycnocline of the upper $50 \mathrm{~m}$. Both the position, strength, and depth of the jet changed between each successive crossing. Estimates of vorticity, approximated by the along-track 
horizontal gradient of across-track currents, show elevated values greater than the planetary vorticity $f$ in the first and third transects (Fig.3-7B), in a narrow, $10 \mathrm{~km}$ wide section. The rapid timescale of flow evolution, and the strong vorticity, indicate that the narrow jet is submesoscale in its dynamical character (Capet et al., 2008b).
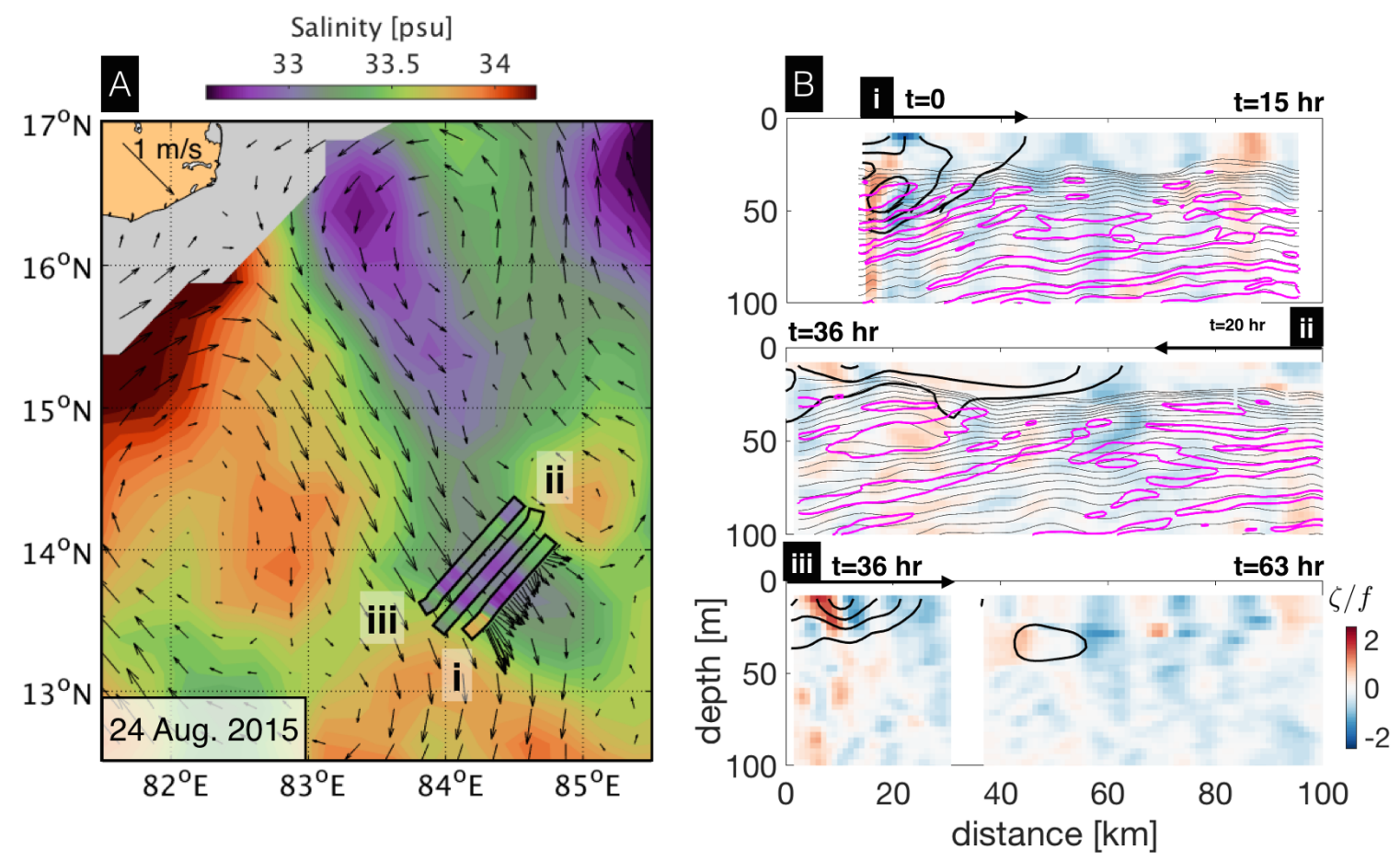

Figure 3-7: Repeat transects across the fast jet shown in the inset of Fig.3-1. (A) Surface geostrophic currents (arrows) and surface salinity (color) measured by satellite (background) and the ship (transects i, ii, and iii, the latter two have been shifted NW in the plot) between August 24 and 27, 2015. Note that the surface density front (dominated by the salinity gradient) is oriented opposite to the implied density gradient at depth that is balanced by the mesoscale flow. (B) Background color shows the vorticity component $U_{y}$ (alongtrack gradient of across-track velocity) normalized by planetary vorticity $f$. Thick black lines contour current speeds above $50 \mathrm{~cm} \mathrm{~s}^{-1}$ in $10 \mathrm{~cm} \mathrm{~s}^{-1}$ increments. Thin lines mark isopycnals in $0.2 \sigma$ increments. Magenta lines contour the layers of negative Diapycnal Spiciness Curvature $\tau_{\sigma \sigma}$. Note the significant changes in jet locations and strengths between each of the 3 transects taken within 1.5 days of each other, less than one inertial period. FCTD $T S$ profiling was conducted only during the first two transects.

A sheared, recirculating submesoscale eddy at a spice front would quickly produce layering. Illustrated by a kinematic model in Fig.3-8A, the rotating, vertically sheared currents (decaying in magnitude with increasing depth), simply tilt and twist the pre-existing spice front by multiple rotations, producing a new watermass layer with each turn. To test the viability of this mechanism, an idealised but dynamic simulation was made with a numerical 
finite-volume fluid dynamics model, Process Study Ocean Model (PSOM) (Mahadevan et al., 1996). The following configuration is heuristic, and not representative of the real ocean. A sharp density front, with a coincident spice gradient, was set up in a periodic channel, with an across-channel (across-front) width of $192 \mathrm{~km}$ between solid boundaries, and an alongchannel (along-front) length of $96 \mathrm{~km}$ (Fig.3-8B) between periodic boundaries. The model had a 64-level vertical grid, with a stretched coordinate with average vertical cell thickness increasing with depth, varying from about $3 \mathrm{~m}$ near the surface to $5 \mathrm{~m}$ at $100 \mathrm{~m}$ depth, and coarser below. The initial density front was created by horizontally interpolating between two $T S$ depth-profiles with a tanh function. The two profiles have the same $T S$ values at depths below $100 \mathrm{~m}$, but have a difference in density, and watermass, above $100 \mathrm{~m}$, that increases toward the surface. Below a $30 \mathrm{~m}$ deep mixed layer, the interpolation between the two profiles produces a pycnocline with sharply tilted isopycnals (Fig.3-8B). To encourage quick development of submesoscale instabilities, the sharpness of the front is somewhat exaggerated, with negative Ertel potential vorticity values. This density front is initially balanced by a geostrophic along-front current, narrowly focused in a jet that decays with depth. The $1 \mathrm{~km}$ horizontal resolution of the simulation allows submesoscale vortices to develop within the larger-scale flow, but does not resolve smaller submesoscale instabilities (Mahadevan, 2006).

As Fig.3-8B shows, a small eddy forms within a few inertial periods and stirs water parcels across the front. The shear below the surface-intensified current (Fig.3-8C) produces several layers of alternating spice below the front, prominently visible in DSC (Fig.3-8E). Furthermore, while the motion that produces the spice layering can be purely along-isopycnal and geostrophic, the eddy's horizontal and vertical shear (Fig.3-8F) in the simulation produces critical Richardson numbers (Fig.3-8H) and $O(1)$ Rossby numbers (Fig.3-8G), thus ageostrophic components are likely not negligible. The eddy's non-geostrophic currents can slump isopycnals. At the location where the submesoscale eddy currents push water from the lighter to the denser side of the front, isopycnals are slumped, and stratification is enhanced (Mahadevan et al., 2012). This leads to layers of anomalous density stratification coincident with spice layering (Fig.3-8B). This mechanism of submesoscale eddy twisting could be the source of the shallow layering observed in the 'jet' area, underneath the submesoscale jet at the SLA and density front, given the expectation that similar dynamics could arise there. While this simulation supports the viability of submesoscale eddies producing layering, and 


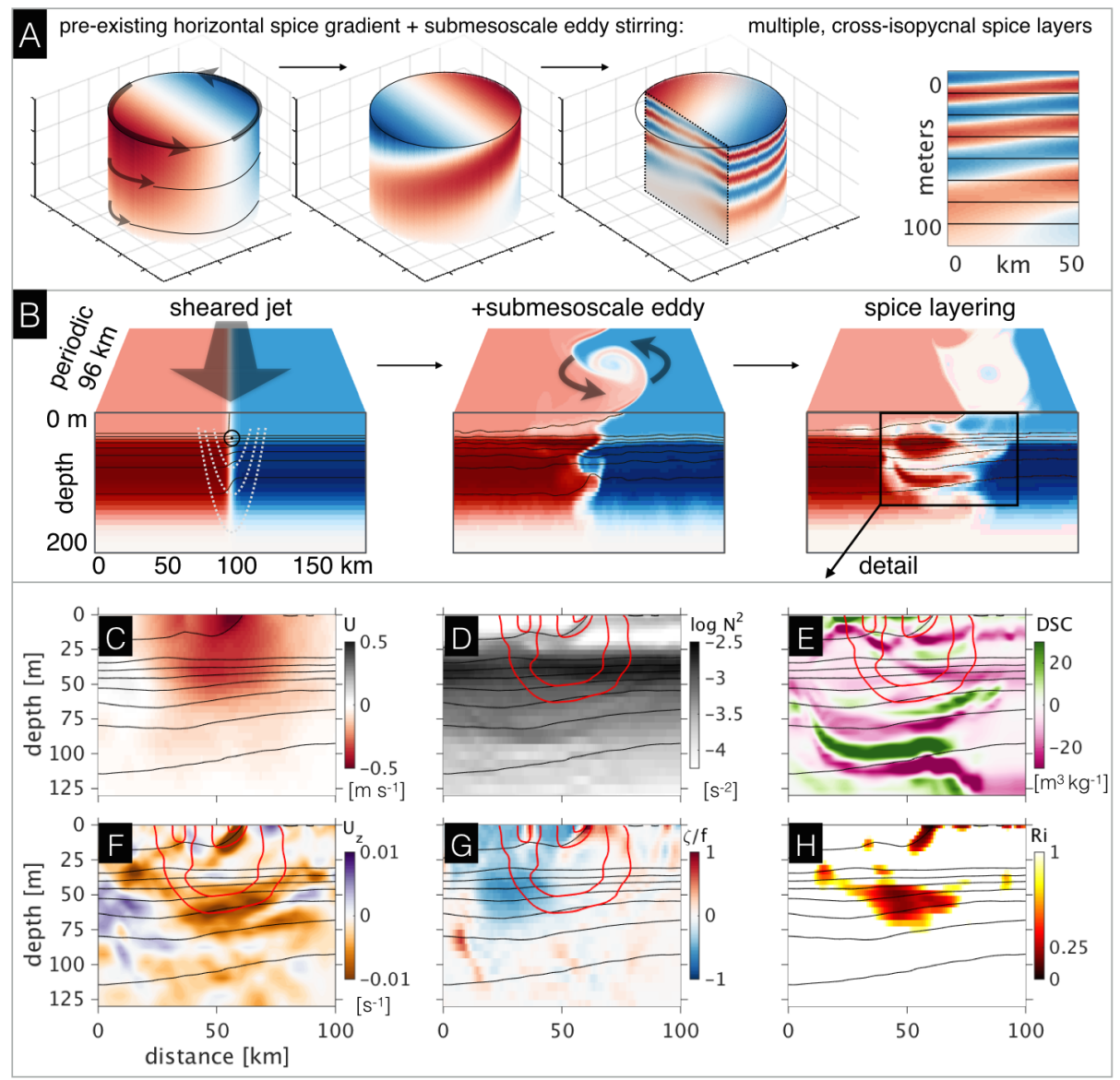

Figure 3-8: Schematic of how a submesoscale eddy along a watermass and density front can create spice interleaving. (A) Mechanistic sequence of the process: An along-isopycnal gradient of spice (red to blue) is advected by along-isopycnal currents that are vertically decaying with depth. The sheared currents tilt the spice front, and after multiple rotations, multiple coherent layers of alternating watermass are formed that cross isopycnals. Their slope decreases with time, and depends on depth and the location of the transect relative to the eddy center. (B) Idealized numerical model simulation of a submesoscale eddy at a density front, creating interleaving. The initial set-up of a density (and spice) front in a 200 $\mathrm{km}$ wide channel leads to a sheared along-front jet, which develops instabilities that grow into a submesoscale eddy. The combination of across-front circulation and vertical shear produces interleaving layers of spice. Black rectangle marks the position of the following detail sections. (C) Along-channel (across-section) velocity. Red color marks out-of-page flow, black lines mark isopycnals in $1 \mathrm{~kg} \mathrm{~m}^{-3}$ increments, also shown in the following panels. (D) $\log _{10}$ of density stratification $N^{2}$. Red contours mark across-section current magnitudes in $10 \mathrm{~cm} \mathrm{~s}^{-1}$ increments, also shown in the following panels. (E) Diapycnal Spiciness Curvature. Note the coincidence of DSC and $N^{2}$ anomaly layers. (F) Vertical shear, acrosssection component. (G) Rossby number, calculated as vorticity normalized by the Coriolis parameter. (H) Richardson number, defined as $N^{2} /$ Shear ${ }^{2}$. Smaller values around 0.25 are indicative of enhanced shear production of turbulence. 
reproduces multiple parallel $\sim 50 \mathrm{~km}$ long spice and DSC layers of similar character as the observations, it should be taken heuristically, rather than as a true representation of the flow environment in the BoB. Furthermore, the deeper layering in the 'jet' section, with at least $80 \mathrm{~km}$ extent, is unlikely to have also been created by an eddy at submesoscales. Instead, the following (second) proposed mechanism could be responsible.

\subsubsection{Hypothesized Mechanism B: Near-Inertial Wave Shear}

The 'shear' area in the transect, unlike the 'jet' section, does not contain fast currents, but is distinguished by prominent vertical shear layers that are around $10 \mathrm{~m}$ thin, nearly flat, and extending over $80 \mathrm{~km}$ length. The layers, visible as changes in shear direction in Fig.3-2B, or as alternating anomalies in $U_{z}$ and $V_{z}$ (vertical gradient of across- and along-track current) in Fig.3-3, extend from below the surface mixed layer to at least 150 m depth. In the upper $100 \mathrm{~m}$, they are coincident and parallel with the observed layers of spice. To clarify the relation between shear and spice, Fig.3-9B shows a hodograph of an average shear profile (horizontally averaged over $10 \mathrm{~km}$ within region \#4) of the two components $U_{z}$ and $V_{z}$, colored by spice. The shear is neither rotating clearly clockwise nor counter-clockwise, but dominated by oscillations along one diagonal axis. The spice anomaly oscillates from positive at one end of the diagonal axis, to negative at the other, coherent with the shear anomalies.

This leads us to the second proposed mechanism that produces multiple parallel spice layers, oscillatory horizontal stirring, specifically by near-inertial waves (NIWs) with a high vertical wavenumber acting on a pre-existing horizontal spice front. Illustrated by a kinematic model in Fig.3-9A, the vertically sheared flow, oscillating at each depth level in a clockwise progression of flow direction, simply interleaves the two watermasses at the spice front in layers of the same thickness as the vertical wavelength. Whether a section through this area reveals interleaving or parallel layering depends on the orientation of the section relative to the orientation of the spice front; a section along the front reveals multiple parallel layers in spice, a section across the front reveals interleaving of spice. The magnitude of the alternating spice anomaly in the vertical depends on the sharpness of the pre-existing horizontal spice front, and the horizontal displacement by the NIW flow. In the 'shear' section detail (Fig.3-3), the vertical spice anomalies are of the same magnitude as the horizontal change in spice seen in the same section across a $\sim 10 \mathrm{~km}$ gradient, both covering a spice 
range of about $0.2 \mathrm{~kg} \mathrm{~m}^{3}$. We estimate the relative horizontal excursion between alternating shear layers by multiplying one half the inertial period $(0.5 \cdot \sim 2$ days) by the relative speed between adjacent layers $\left(O(0.1) \mathrm{m} \mathrm{s}^{-1}\right.$, shear magnitude multiplied by the vertical wavelength), which gives about $O(10) \mathrm{km}$ horizontal displacements. The NIW interleaving can thus produce vertical spice anomalies of the observed magnitude.

And while the oscillatory flow is mostly horizontal, the wave's vertical component will nonetheless induce alternating anomalies of vertical strain (Alford et al., 2016). The vertical displacement needed to produce the observed magnitude of stratification anomalies is estimated by decomposing the vertical gradient of density (approximated to be smoothly increasing with depth) into a background and a wave strain component, such that $N^{2}=$ $\left(-g / \rho_{o}\right) \frac{\partial \rho(z)}{\partial z}\left[1+\frac{\partial}{\partial z} \xi\right]$, where $\xi$ is the vertical displacement due to the NIW, and its vertical derivative is strain. Relative stratification anomalies of $20 \%$, observed for example in the BoB sections, are produced by alternating vertical displacements of $2 \mathrm{~m}$ over $10 \mathrm{~m}$ vertical wavelengths, or strain $\xi_{z}=0.2$. Such strain values are consistent with linear internal wave scaling of strain as $\sim \frac{1}{\omega}\left(K_{x} U+K_{y} V\right)$, (Sun and Kunze, 1999) applied to near-inertial frequencies of $\omega \sim O\left(10^{-5}\right) \mathrm{s}^{-1}$, horizontal wavelengths of $O(100) \mathrm{km}$, and relative horizontal velocities of $O(0.1) \mathrm{m} \mathrm{s}^{-1}$ in the 'shear' section (Fig.3-3). Thus, a low-frequency, highvertical wavenumber wave can produce multiple parallel layers of spice and stratification anomalies. We propose this mechanism as a possible source of the observed layering in the 'shear' section, as well as of the similar layering observed below $75 \mathrm{~m}$ in the 'jet' section.

The source of the observed NIW could be either downward propagating energy from atmospheric wind forcing, or energy radiating from the frontal jet, for example by a loss of balance of the flow (Alford et al., 2013). The atmospheric forcing during the AugustSeptember 2015 cruise, in the late summer monsoon season, contained impulsive and strong wind changes that could have stimulated NIWs. The forcing was marked by both weak $(<5 \mathrm{~m}$ $\mathrm{s}^{-1}$ ) and robust $\left(>10 \mathrm{~m} \mathrm{~s}^{-1}\right)$ wind speeds, generally from a southwesterly direction, though with gales from other directions too. A rotary spectral decomposition of vertical shear profiles (Rainville and Pinkel, 2004) collected during the cruise, shows that on average, clock-wise oscillations dominate, with vertical scales of $O(10) \mathrm{m}$ (Fig.3-9, indicative of downward propagating surface-forced NIWs. Furthermore, NIWs were separately observed in the BoB by (Johnston et al., 2016) from glider observations.

To identify and distinguish these two potential layering mechanisms in action, hydro- 
graphic observations are needed that track layers over time, ideally for at least a full inertial period. Sub-surface waters exhibiting a layering pattern could be sampled with short sections of vertical profiles of $T, S$, and shear, and followed as the layers are potentially advected by mesoscale flows and tides. Such observations would reveal whether the spice layers reverse and evolve with a coincident near-inertial oscillation, whether they are independent of NIW shear, but increase in wavenumber as a surface eddy adds twists of alternating watermass from above, or whether they are independent of both NIW and eddy rotations, evolving only slowly as a passive feature sheared by mesoscale flows.

\subsection{Discussion}

The two mechanisms, NIW shear and submesoscale sheared eddy twist, produce thin long layers on a shorter timescale than mesoscale stirring. The predicted scales of horizontal extent, vertical thickness, formation time, and spice anomaly magnitude, are summarized in Fig.3-10.

The properties of layers formed by submesoscale sheared eddy twist are time dependent. Every full rotation of an eddy increases the number of layers by one, between the surface and the depth at which the eddy current is negligible. The formation timescale of $n$ layers is dependent on the eddy rotation rate, estimated by the width of the eddy $(L)$ divided by the maximum current magnitude $\left(U_{\max }\right)$. The resultant scale, $\sim n L / U_{\max }$, can also be expressed as a function of vorticity as $\sim n / \zeta$, by approximating the vorticity magnitude as $U_{\max } / L$. Eddy currents with higher vorticity create layers more rapidly.

Based on the observed characteristics of the shallow layering in the jet section in the BoB (Fig.3-7), with $\sim 5$ spice layers beneath a jet with $O(f)$ vorticity, and accounting for a factor of $2 \pi$, the timescale of formation is estimated as $5 \cdot 2 \pi / 4 \times 10^{-5} \mathrm{~s}$, or roughly 10 days. While the lateral layer scale depends on the size of the eddy $L$, the vertical thickness of these layers is proportional to the depth of the eddy $H$ (depth over which the surface intensified currents decay), divided by the number of layers $n$. In the jet observations, with the strong current restricted to the top $\sim 75 \mathrm{~m}$ (Fig.3-7), a $10 \mathrm{~m}$ vertical thickness for $\sim 5$ layers is in line with our scaling estimate of $\sim 75 / 5$, or about $15 \mathrm{~m}$.

In contrast, a mesoscale eddy with smaller vorticity would need an order of magnitude longer to form multiple vertical layers by twisting an existing horizontal spice gradient. For 


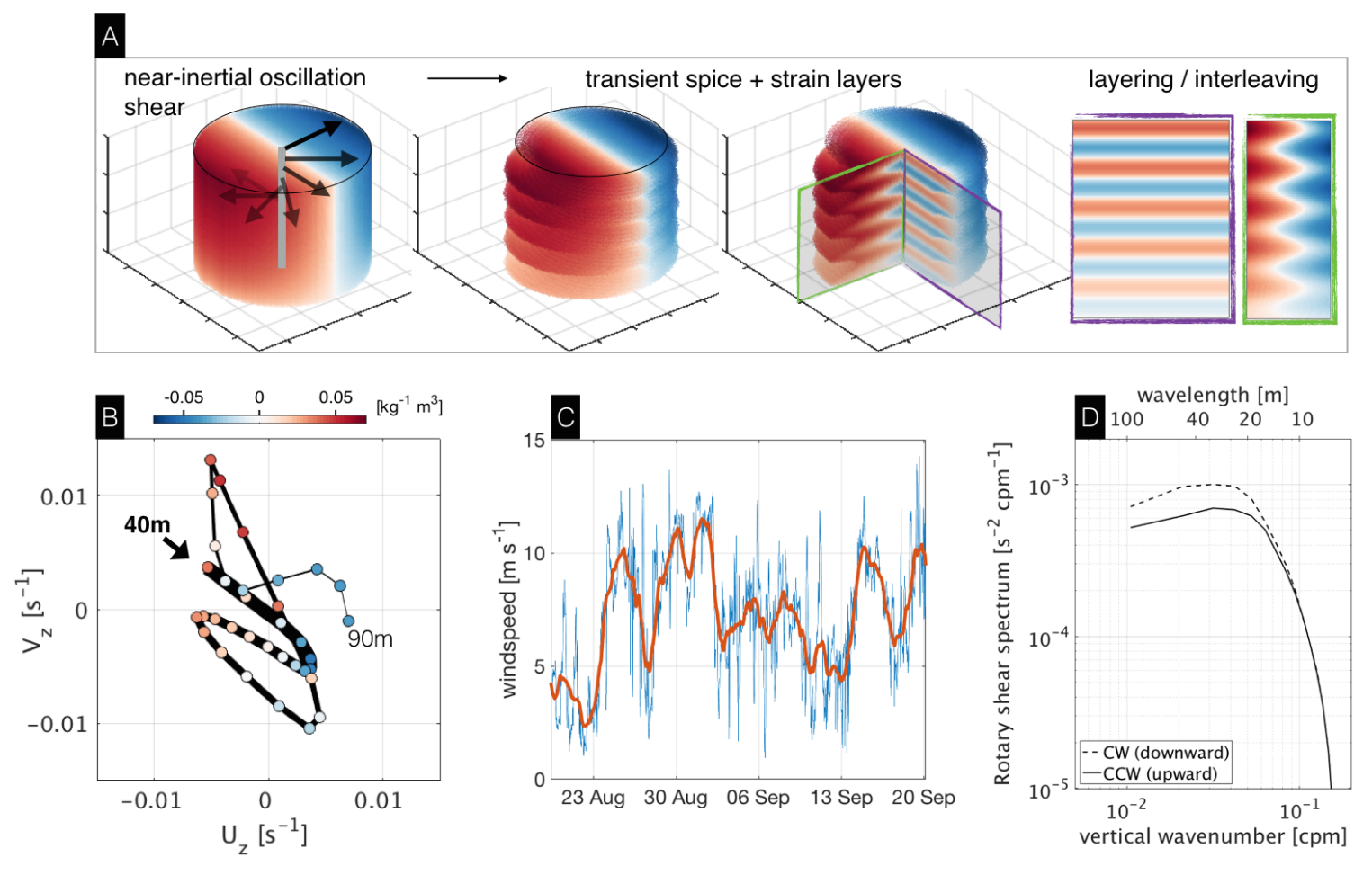

Figure 3-9: Schematic of how a vertically sheared oscillation at a watermass front can create spice interleaving. (A) Mechanistic sequence of the proposed NIW spice interleaving process: An along-isopycnal gradient of spice (red to blue) is advected by along-isopycnal motions in different directions depending on depth. Depending on the transect orientation relative to the spice front orientation, either interleaving wiggles or horizontal layers of spice are observed. The vertical wavelength of the wave sets the vertical wavelength of the spice layering. (B) Hodograph of a vertical shear profile through the "Shear Area" section (location marked by black arrow in Fig.3-3), colored by spice $(\tau)$. The thickness of the black line indicates the depth along the profile, going from $40 \mathrm{~m}$ (thick) to $90 \mathrm{~m}$ (thin). (C) Wind speed encountered during the 2015 cruise. Blue line shows the hourly mean, orange line the 24 hour mean wind speed. The prevailing direction was southwesterly, though individual squalls and rainstorms blew from other directions too. The 'Shear' section was made at the very end of the cruise. (D) A spectral decomposition of shear in vertical wavenumber space, measured by a shipboard $140 \mathrm{kHz}$ HDSS acoustic Doppler sonar system during the 2015 cruise, reveals an excess of clock-wise rotating components, indicative of downward propagating near-inertial oscillations.

example, an eddy $200 \mathrm{~km}$ across with current magnitudes of $0.5 \mathrm{~m} \mathrm{~s}^{-1}$ decaying linearly over the top $100 \mathrm{~m}$, would need $\sim 100$ days to create 4 layers of $25 \mathrm{~m}$ vertical thickness each. Submesoscale eddies with $O(1)$ or larger Rossby numbers create layering an order of magnitude faster than mesoscale eddies.

Layers created by the sheared eddy twist mechanism have a thickness $H / n$ that decreases with time, inversely proportional to the formation timescale. In the above example, the 
submesoscale jet created $\sim 10 \mathrm{~m}$ thick layers in about 10 days, and would create $\sim 5 \mathrm{~m}$ thick layers in 30 days, or $\sim 1 \mathrm{~m}$ thick in $2-3$ months. The thinness might be limited by the scale of isotropic turbulent mixing events, such as breaking internal waves, independent of a (low) time-mean value of diffusivity observed in the BoB.

Unlike the time-dependent properties of layers created by the sheared eddy twist mechanism, the properties of NIW shear induced layers are closely and simply related to the wave characteristics. Layers with vertical thickness equal to the vertical shear wavelength, and lateral extent equal to the NIW lateral extent (or, if shorter, the spice front length), form on the timescale of the local inertial period $\sim 2 \pi / f$, or about 2 days in the BoB. Of course the interleaving is theoretically reversed a half-period later, however with the superposition of other currents and shears, the watermass layering does not necessarily fully reverse.

\begin{tabular}{|c|c|c|}
\hline Layering & Near inertial wave shear & Submesoscale sheared eddy twist \\
\hline Lengthscale $\Delta \mathrm{x}$ & $\begin{array}{l}\text { horizontal wavelength, } \\
\text { or length of spice front }\end{array}$ & diameter of eddy $\sim \mathrm{L}$ \\
\hline Timescale & inertial period $\sim 1 / \mathrm{f}$ & $\begin{array}{l}1 \text { layer per rotation: } \\
\mathrm{N}_{\text {layers }} \cdot \text { Period }_{\text {rotation }} \approx\left(\mathrm{N} \cdot \mathrm{L} / \mathrm{U}_{\max }\right)\end{array}$ \\
\hline Thickness $\Delta z$ & vertical wavelength & $\begin{array}{l}\text { eddy depth } \mathrm{H} \cdot 1 / \mathrm{N}_{\text {layers }} \\
\quad \approx \mathrm{H} \cdot \mathrm{L} /\left(\mathrm{U} \cdot \mathrm{t}_{\text {elapsed }}\right)\end{array}$ \\
\hline Spice anom. $\Delta_{\mathrm{v}} \tau$ & $\sim \nabla_{\text {hor }} \tau \cdot \mid$ Shear $\mid \cdot 1 / \mathrm{f}$ & $\sim \nabla_{\text {hor }} \tau \cdot \mathrm{L}$ \\
\hline
\end{tabular}

Figure 3-10: Table summarizing the predicted properties of tracer layering produced by the proposed new mechanisms: interleaving by NIWs (Fig.3-9A), and layering by a submesoscale sheared eddy twist (Fig.3-8A).

\subsubsection{Implications for Mixing in the Bay of Bengal}

The prominent spice layering observed in the $\mathrm{BoB}$, characterized by multiple thin parallel layers in DSC, stratification, and shear, suggests that submesoscale and NIW phenomena are prevalent, and play a central role in stirring the upper ocean. The observed spice and shear layering, with only $10 \mathrm{~m}$ vertical wavelengths (but at least $80 \mathrm{~km}$ horizontal extent in one instance), draws attention to NIWs with a much higher wavenumber than the majority of NIWs studied (Alford et al., 2016). Near-inertial oscillations were proposed (Beal, 2007) as a source of interleaving in the Agulhas front, but for spice layers an order of magnitude thicker. Yet higher-wavenumber waves might be especially effective at contributing to shear 
dispersion, since the thinner layers can be vertically homogenized by turbulent mixing more rapidly than thicker layers would be. During the cruise of August-September 2015, vertical shear was observed to contain significant downward-propagating modes with $O(10) \mathrm{m}$ vertical wavelengths. Perhaps a larger than usual portion of energy input by winds into NIWs in the $\mathrm{BoB}$ is contained in high-vertical-wavenumber modes, increasing the effectiveness of shear dispersion on horizontal mixing (Young et al., 1982), and potentially its (usually small) importance relative to dispersion by submesoscale eddies.

The large aspect ratio (near-flat slopes) of the prominent layers, of $O(10) \mathrm{m}$ thickness and perhaps up to $\sim 100 \mathrm{~km}$ length, implies that diapycnal mixing, however weak, is effectively mixing at much larger horizontal scales when it vertically mixes such gently sloping features (Sundermeyer and Ledwell, 2001). If the source mechanism of these layers were mesoscale lateral strain and vertical shear (Haynes and Anglade, 1997), or small-scale strain and shear (Smith and Ferrari, 2009), the thinness of the layers would indicate very weak turbulent mixing that permits tracer features to cascade to high vertical wavenumbers before being mixed. Indeed observations of turbulence in the BoB reveal very weak average mixing levels (Shroyer et al., 2016b).

Yet if the layering, instead of being produced by background shear and strain, is produced by either the submesoscale twist or the NIW layering mechanism, the formation timescale is also much shorter, on the order of inertial periods. In this case, the thinness and extended horizontal coherence of the layering is not necessarily enabled by low vertical mixing levels, but instead presents a direct route for diapycnal mixing to effectively mix at much larger horizontal scales.

The flatter slopes of $O\left(10^{-4}\right)$ imply a tenfold increase in horizontal scale at which turbulent mixing effectively reduces horizontal gradients by shear dispersion, compared to regimes where only mesoscale stirring creates sloping tracer features with average slopes of $O\left(10^{-3}\right)$. And if these submesoscale or NIW processes also incidentally enhance vertical mixing by increasing fine-scale shear (Garrett and Munk, 1972), then the formation of these thin layers represents a potential source of horizontal stirring (and ultimately mixing) at sub-mesoscales, in addition to dispersion driven by mesoscale currents in the BoB. Submesoscale and NIW dynamics are potentially the rate-controlling processes for the formation of vertical gradients.

Of further interest in the 'jet' section is the presence, in relative proximity to the surface 
jet, of deep spice layering, perhaps created by NIW shear, given its similarity to the 'shear' section described above. This raises two additional possible interpretations of the layering in this region, and questions for further study. The implied NIW could have been directly forced by energy radiating from the front (Alford et al., 2013). We indeed observe timedependence of the narrow and fast current, potentially transferring energy from balanced motions to waves. Additionally, the shallow layering below the front could also have been caused by a NIW, but altered and steepened by interactions between the oscillations of the wave and the baroclinic submesoscale flow (Whitt and Thomas, 2013). The large vorticity values, both positive and negative, surrounding the jet could alter the propagation and characteristics of the NIW, which caused the cross-isopycnal layering.

Interesting questions remain about NIW modes with very high vertical wavenumbers, specifically regarding their generation and interactions. The role of the unusually strong and shallow density stratification in modulating surface near-inertial forcing, the potential source of NIWs by adjustments of frontal jets, and the interactions of NIWs with high-vorticity submesoscale fronts, are topics that would benefit from further modelling simulations and observational work in the BoB.

\subsection{Summary}

Observations from the upper $200 \mathrm{~m}$ of the Bay of Bengal over several 20-100 km long sections reveal thermohaline patterns of multiple parallel $\sim 10 \mathrm{~m}$ thin layers with a shallow cross-isopycnal slope of $O\left(10^{-4}\right)$. Along with the watermass layering, visible as spice or as DSC anomalies, we observe coincident and parallel density stratification anomalies and vertical shear layering. The characteristics of these patterns lead us to propose two specific mechanisms previously not considered in the formation of thermohaline layering of this type. The layering can either be the signature of a submesoscale sheared eddy, stirring an existing spice front by twisting it around, or it can be the signature of a low-frequency (near inertial), high-wavenumber internal wave, with predominantly horizontal flow, vertically interleaving an existing horizontal spice front. The thinness, the large lateral extent, and the relatively short formation timescale of these watermass layers, implies that vertical diapycnal mixing can contribute to effective horizontal tracer mixing at scales larger than it would otherwise act over in the absence of these layering processes. 
THIS PAGE INTENTIONALLY LEFT BLANK 


\section{Conclusion}

This thesis provides answers to the dual questions posed by the ASIRI - MISO-BOB - OMM research initiatives: What specific ocean dynamics control the Bay of Bengal's behaviour? And what does the behaviour of the Bay of Bengal tell us about upper ocean dynamics in general?

These questions were approached with a combination of data analysis, heuristic toy models, idealised numerical simulations, and simple theoretical scaling. The starting points for these inquiries, in each of the three chapters, were patterns of temperature and salinity that caught our attention amidst the trove of observations collected during several research cruises in the BoB. In the surface through-flow data, we noticed an unexplained correlation between temperature and salinity. Beneath the fresh surface layer, a complex field of salinity variance. Crossing isopycnals, a strikingly detailed pattern of parallel sloping watermass and stratification layers. These hydrographic observations are connected by common threads: they are only visible because of the competing (and thus compensating) influence of salinity and temperature gradients in the $\mathrm{BoB}$, and they are signatures of the turbulent flow dynamics shaping the upper ocean conditions.

Dominating surface buoyancy fields, the strong and often sharp salinity gradients are an effect of the annual input of fresh water in the BoB, supplied by monsoon rains and river runoff. The freshwater, significantly strengthening the upper ocean's stratification, is stirred around by ocean currents and waves at both meso- and submeso-scales. Figure 4-1 schematically presents some of the key connections between monsoon freshwater and the dynamics in the BoB. The work presented in this thesis revealed some of the controlling processes behind the stirring and stratification of freshwater in the $\mathrm{BoB}$, and furthers our understanding of this unique oceanic region and its links with the monsoon. 


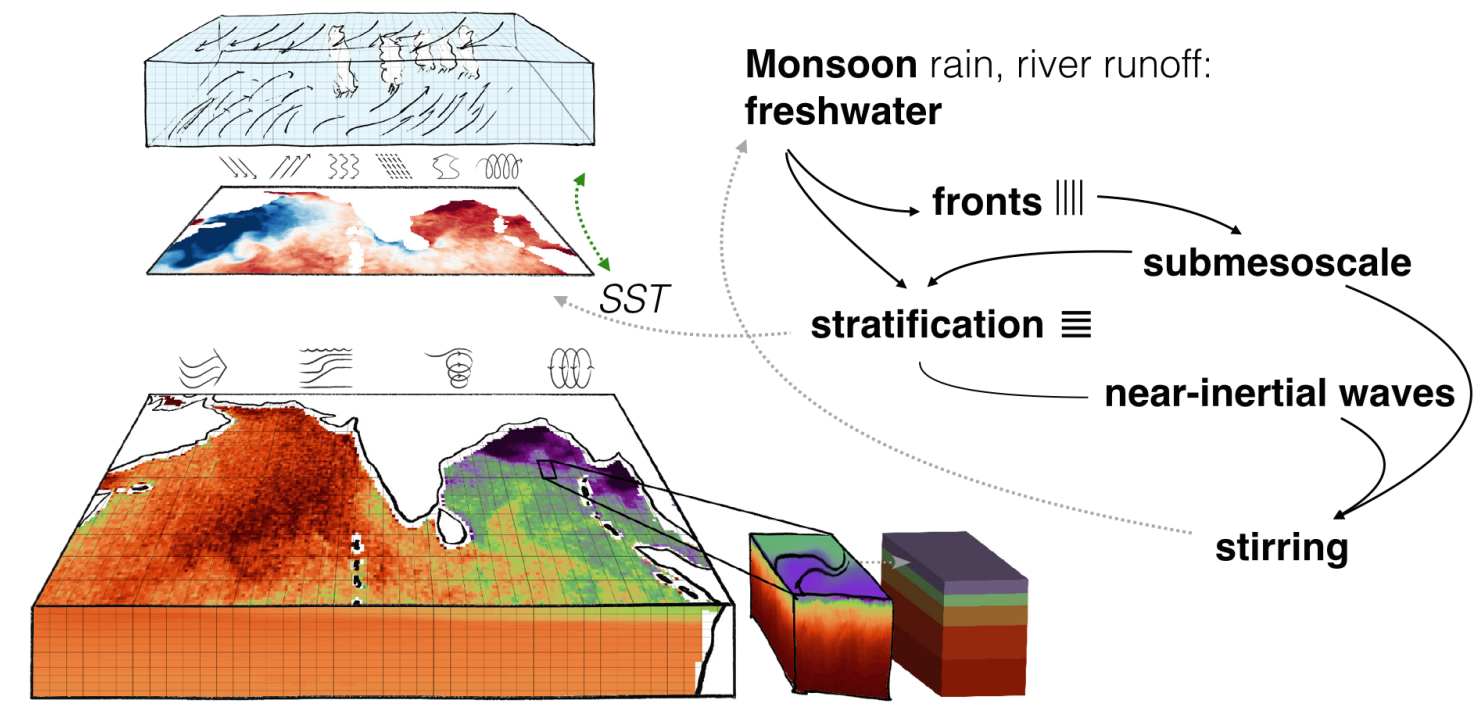

Figure 4-1: Schematic interconnecting the Monsoon freshwater forcing and the associated dynamical, hydrographic, and thermodynamic effects and processes in the Bay of Bengal.

Chapter 1 (Jaeger and Mahadevan, 2018) presents the discovery of small-scale thermohaline compensation in an otherwise large-scale salinity-gradient dominated surface layer. Data analysis of sub-surface hydrographic profiles, and air-sea flux measurements, suggests that the mechanism behind the surface compensation pattern involves a combination of surface mixed layer submesoscale dynamics and atmospheric forcing. Idealized numerical simulations support this interpretation, reproducing the submesoscale-selective compensation of fronts in a salinity-stratified ocean. The observed thermohaline pattern thus reveals the activity of submesoscale re-stratification in the Bay of Bengal, which shoals the surface mixed layer and suppresses vertical ventilation. The findings emphasize the role salinity gradients can have on density, an important fact to consider when interpreting cold SST filaments in satellite images, since these do not necessarily mark dense filaments if produced by the mechanism described here. Furthermore, by selectively cooling submesoscale salinity fronts, and so reducing small-scale density gradients, the mechanism weakens the further growth of submesoscale instabilities, and potentially the associated energy cascade. Similar compensation is estimated to be encountered in other regions of the oceans, such as the Arctic or river plumes in coastal areas that are influenced by freshwater runoff and subjected to strong cooling from the atmosphere. 
Chapter 2 further investigated thermohaline compensation signals, but below the surface mixed layer of the Bay of Bengal, analyzing along-isopycnal water mass anomalies and their scale-dependent distribution. It is the first such analysis in the Indian Ocean. What did this large hydrographic dataset, collected during 3 research cruises in 2013, 2015, and 2018, reveal about the sub-surface dynamics? Synthesizing wavenumber statistics along almost $5000 \mathrm{~km}$ of hydrographic sections, assembled from over 9000 vertical temperature and salinity profiles of the upper $200 \mathrm{~m}$, the results provide three answers. (i) There are distinct dynamical stirring regimes controlling stirring at $\sim 50 \mathrm{~m}, 100 \mathrm{~m}$, and $200 \mathrm{~m}$ depths. (ii) The tracer patterns support quasigeostrophic (QG) predictions only below $100 \mathrm{~m}$ depth and at the largest $(100 \mathrm{~km})$ scales; at shallower depths or smaller scales, surface QG or ageostrophic frontogenesis appear to dominate. (iii) Within layers shallower than $100 \mathrm{~m}$, at scales smaller than $10 \mathrm{~km}$, there is an unexpected lack of small-scale variance. The discovery of this spectral-slope change suggests that extra stirring, and effectively horizontal mixing, occurs at $1-10 \mathrm{~km}$ scales in the highly stratified layers below the surface mixed layer. The various observed spectral slopes also raise questions about the validity of some of the common assumptions made by theoretical predictions of ocean stirring. While far removed from bottom topography effects, the tracer and current field is very possibly forced by surface instabilities at $O(1-10) \mathrm{km}$ scales, the assumption of an inertial range in the tracer cascade might thus be flawed. Further work is needed to confidently interpret tracer spectral distributions from observations. A useful set of heuristic simulations could analyze variance spectra of tracer stirred by mesoscale currents, either resolving or smoothing submesoscale flows and either permitting or suppressing near-inertial waves.

Motivated by the questions raised by the horizontal scale distributions, we further analyzed the spatial distribution of spice in the observed sections, specifically extending to vertical variability and 2-dimensional patterns. In the hope of finding clues of the extra stirring and mixing necessitated by the spectral results of Chapter 2, our analysis of spice layering focused on patterns with thin $\sim 10 \mathrm{~m}$ vertical scales yet large $O(10) \mathrm{km}$ lateral extent, and proposes two potential stirring candidates. We speculate that the stirring sources are either submesoscale vortical modes, or shear dispersion by near-inertial waves, the latter potentially enhanced by high vertical wavenumber modes encountered in the Bay of Bengal's sharply stratified upper ocean. 
Chapter 3 analysed water mass patterns sloping across isopycnals, specifically in regions with prominent multiple parallel layers coherent over 10's of kilometers. The unusually shallow slopes of these layers, as well as coincident shear and density stratification anomalies, lead to two new mechanisms of tracer layering: submesoscale sheared eddy stirring, and near-inertial wave shear. The proposed mechanisms act on pre-existing lateral spice gradients, producing layers in much shorter time than mesoscale stirring, and with a much longer-and-thinner aspect ratio. Idealized numerical modeling of a submesoscale eddy reproduces layering very similar to the observations, and an analysis of shear profiles finds a signature of downward propagating oscillations with vertical wavelengths of order 10 meters. The result suggest that these mechanisms could enhance effective horizontal mixing through stirring and shear dispersion, and increase the horizontal scale at which vertical mixing is effectively felt, to $1-10 \mathrm{~km}$.

The chapters of this thesis investigated thermohaline patterns, finding signatures of submesoscale instabilities, eddies, and near-inertial waves. The work illuminates the dynamics acting below the surface of the Bay of Bengal, a soup constantly stirred even though highly stratified. The influence of submesoscale dynamics, seen in the shallow restratification and thermohaline compensation mechanism of Chapter 1, extends below the surface mixed layer. Down to a depth of almost $100 \mathrm{~m}$, below the peak density stratification, tracer variance spectra indicate the presence of enhanced stirring at submesoscales. A change in spectral slope near $10 \mathrm{~km}$ scales is indicative of a change in the rate-controlling process cascading tracer variance downscale. This change in spectral slope, sharpest in the spectra of the shallowest layer, smoother in the slightly deeper spectra, and not seen deeper down between 100 and $200 \mathrm{~m}$ depth, is suggestive of a near-surface process that penetrates into the top of the pycnocline. In addition to submesoscale eddies, we propose near-inertial waves with high vertical wavenumbers as another viable candidate contributing to tracer stirring at $1-10 \mathrm{~km}$ scales. The depth-dependence in that case could arise from the stretching of vertical wavelengths with decreasing stratification, reducing the efficiency of NIW shear dispersion mixing as interleaving layers become thicker and vertical tracer gradients weaker. Both processes, submesoscale eddies and NIWs, are likely modulated by the strong near-surface density stratification, highlighting the influence of the monsoon freshwater on ocean dynamics in the Bay of Bengal. 
Returning to the over-arching goal of ASIRI - MISO-BOB - OMM, our results have improved our understanding of the Bay of Bengal's surface conditions and salinity stratification, thereby pointing at potential challenges that need to be addressed to improve coupled oceanatmosphere representations of the Monsoon system. Evidence of submesoscale circulations re-stratifying the surface boundary layer, in Chapter 1, emphasizes the need to either explicitly resolve, both horizontally and vertically, or carefully parametrize, frontal instabilities producing very shallow surface mixed layers. Furthermore, the evidence for submesoscale turbulence horizontally stirring below $10 \mathrm{~km}$ scales, while not directly contributing to diapycnal mixing or restratification, has the potential to affect watermass dispersal and mixing dynamics via vorticity anomalies.

It is clear that two-dimensional macro-turbulence in the ocean, especially at submesoscales, is not yet completely understood. Areas of particular uncertainty and intrigue are the interactions between sub-inertial instabilities and near-inertial oscillations. Both of these phenomena were observed in the Bay of Bengal with similar horizontal spatial scales, and both are viable candidates for the stirring (and ultimately mixing) implied by the spectral tracer variance fall-off in Chapter 2, and the stirring implied by the tracer layering and interleaving in Chapter 3. Of interest to shear dispersion are the high vertical wavenumbers or thinness of the observed shears, laterally coherent over $\sim 100 \mathrm{~km}$. An open question is whether such modes are favoured or affected by the Bay of Bengal's atypical stratification profile, characterized by very strong and shallow pycnoclines, and frequently by additional re-stratification layers above the permanent pycnocline. Equally intriguing is the possibility that some of the shallow interleaving shears, described in Chapter 3, were produced by instabilities in the baroclinic mesoscale current focused in a narrow submesoscale jet. Understanding and parametrizing these phenomena is important if we want to increase the fidelity of ocean models.

Finally, the conclusions reached by this thesis underscore the importance of exploratory research and observations as a valuable addition to strictly hypothesis-driven fieldwork. Focused on a region with a multitude of interesting phenomena, from a variety of atmospheric and oceanic perspectives, and with unique conditions extending the parameter space of typical observational settings, such field-campaigns can yield unexpected insights into fundamental ocean dynamics. The Bay of Bengal is a valuable testing ground for submesoscale dynamical theories because of its unique freshwater influence on upper ocean structure. 
THIS PAGE INTENTIONALLY LEFT BLANK 


\section{Bibliography}

Alford, M. H., MacKinnon, J. A., Simmons, H. L., and Nash, J. D. (2016). Near-inertial internal gravity waves in the ocean. Annual Review of Marine Science, 8:95-123.

Alford, M. H., Shcherbina, A. Y., and Gregg, M. C. (2013). Observations of near-inertial internal gravity waves radiating from a frontal jet. Journal of Physical Oceanography, 43(6):1225-1239.

Badin, G., Tandon, A., and Mahadevan, A. (2011). Lateral mixing in the pycnocline by baroclinic mixed layer eddies. Journal of Physical Oceanography, 41(11):2080-2101.

Batchelor, G. K. (1959). Small-scale variation of convected quantities like temperature in turbulent fluid Part 1. General discussion and the case of small conductivity. Journal of Fluid Mechanics, 5(1):113-133.

Beal, L. M. (2007). Is interleaving in the agulhas current driven by near-inertial velocity perturbations? Journal of Physical Oceanography, 37(4):932-945.

Benshila, R., Durand, F., Masson, S., Bourdallé-Badie, R., de Boyer Montégut, C., Papa, F., and Madec, G. (2014). The upper Bay of Bengal salinity structure in a high-resolution model. Ocean Modelling, 74:36-52.

Birch, D. A., Young, W. R., and Franks, P. J. (2008). Thin layers of plankton: Formation by shear and death by diffusion. Deep Sea Research Part I: Oceanographic Research Papers, $55(3): 277-295$.

Brethouwer, G. and Lindborg, E. (2008). Passive scalars in stratified turbulence. Geophysical Research Letters, 35(6).

Burchard, H. and Bolding, K. (2001). Comparative analysis of four second-moment turbulence closure models for the oceanic mixed layer. Journal of Physical Oceanography, 31(8):1943-1968.

Callies, J. and Ferrari, R. (2013). Interpreting energy and tracer spectra of upper-ocean turbulence in the submesoscale range $(1-200 \mathrm{~km})$. Journal of Physical Oceanography, 43(11):2456-2474.

Callies, J., Ferrari, R., Klymak, J. M., and Gula, J. (2015). Seasonality in submesoscale turbulence. Nature Communications, 6:6862.

Callies, J., Flierl, G., Ferrari, R., and Fox-Kemper, B. (2016). The role of mixed-layer instabilities in submesoscale turbulence. Journal of Fluid Mechanics, 788:5-41. 
Capet, X., Klein, P., Hua, B. L., Lapeyre, G., and Mcwilliams, J. C. (2008a). Surface kinetic energy transfer in surface quasi-geostrophic flows. Journal of Fluid Mechanics, 604:165-174.

Capet, X., McWilliams, J. C., Molemaker, M. J., and Shchepetkin, A. (2008b). Mesoscale to submesoscale transition in the california current system. part iii: Energy balance and flux. Journal of Physical Oceanography, 38(10):2256-2269.

Charney, J. G. (1971). Geostrophic turbulence. Journal of the Atmospheric Sciences, 28(6):1087-1095.

Chowdary, J. S., Srinivas, G., Fousiya, T., Parekh, A., Gnanaseelan, C., Seo, H., and MacKinnon, J. A. (2016). Representation of Bay of Bengal upper-ocean salinity in general circulation models. Oceanography, 29(2):38-49.

Cole, S. T. and Rudnick, D. L. (2012). The spatial distribution and annual cycle of upper ocean thermohaline structure. Journal of Geophysical Research: Oceans, 117(C2).

D’Asaro, E. A., Shcherbina, A. Y., Klymak, J. M., Molemaker, J., Novelli, G., Guigand, C. M., Haza, A. C., Haus, B. K., Ryan, E. H., Jacobs, G. A., et al. (2018). Ocean convergence and the dispersion of flotsam. Proceedings of the National Academy of Sciences, page 201718453 .

de Verneil, A., Franks, P., and Ohman, M. (2019). Frontogenesis and the creation of finescale vertical phytoplankton structure. Journal of Geophysical Research: Oceans.

d'Orgeville, M., Hua, B. L., Schopp, R., and Bunge, L. (2004). Extended deep equatorial layering as a possible imprint of inertial instability. Geophysical Research Letters, 31(22).

Durham, W. M., Kessler, J. O., and Stocker, R. (2009). Disruption of vertical motility by shear triggers formation of thin phytoplankton layers. Science, 323(5917):1067-1070.

Durham, W. M. and Stocker, R. (2012). Thin phytoplankton layers: characteristics, mechanisms, and consequences. Annual Review of Marine Science, 4:177-207.

Ferrari, R. and Polzin, K. L. (2005a). Finescale structure of the t-s relation in the eastern north atlantic. Journal of Physical Oceanography, 35(8):1437-1454.

Ferrari, R. and Polzin, K. L. (2005b). Finescale Structure of the T-S Relation in the Eastern North Atlantic. Journal of Physical Oceanography, 35(8):1437-1454.

Ferrari, R. and Rudnick, D. L. (2000). Thermohaline variability in the upper ocean. Journal of Geophysical Research: Oceans, 105(C7):16857-16883.

Flament, P. (2002). A state variable for characterizing water masses and their diffusive stability: spiciness. Progress in Oceanography, 54(1):493-501.

Fox-Kemper, B., Ferrari, R., and Hallberg, R. (2008). Parameterization of mixed layer eddies. Part I: Theory and diagnosis. Journal of Physical Oceanography, 38(6):1145-1165.

Franks, P. J. (1995). Thin layers of phytoplankton: a model of formation by near-inertial wave shear. Deep Sea Research Part I: Oceanographic Research Papers, 42(1):75-91. 
Garrett, C. (1979). Mixing in the ocean interior. Dynamics of Atmospheres and Oceans, $3(2-4): 239-265$.

Garrett, C. and Munk, W. (1972). Oceanic mixing by breaking internal waves. In Deep Sea Research and Oceanographic Abstracts, number 12, pages 823-832. Elsevier.

Garrett, C. and Munk, W. (1979). Internal waves in the ocean. Annual Review of Fluid Mechanics, 11(1):339-369.

Genin, A., Jaffe, J. S., Reef, R., Richter, C., and Franks, P. J. (2005). Swimming against the flow: a mechanism of zooplankton aggregation. Science, 308(5723):860-862.

Girishkumar, M. S., Ravichandran, M., and McPhaden, M. J. (2013). Temperature inversions and their influence on the mixed layer heat budget during the winters of 20062007 and 2007-2008 in the Bay of Bengal. Journal of Geophysical Research: Oceans, $118(5): 2426-2437$.

Gordon, A. L., Shroyer, E., and Murty, V. (2017). An intrathermocline eddy and a tropical cyclone in the Bay of Bengal. Scientific Reports, 7:46218.

Gordon, A. L., Shroyer, E. L., Mahadevan, A., Sengupta, D., and Freilich, M. (2016). Bay of Bengal: 2013 northeast monsoon upper-ocean circulation. Oceanography, 29(2):82-91.

Grisouard, N. and Thomas, L. N. (2016). Energy exchanges between density fronts and near-inertial waves reflecting off the ocean surface. Journal of Physical Oceanography, 46(2):501-516.

Haynes, P. and Anglade, J. (1997). The vertical-scale cascade in atmospheric tracers due to large-scale differential advection. Journal of the Atmospheric Sciences, 54(9):1121-1136.

Hormann, V., Centurioni, L. R., Mahadevan, A., Essink, S., D’Asaro, E. A., and Kumar, B. P. (2016). Variability of near-surface circulation and sea surface salinity observed from Lagrangian drifters in the northern Bay of Bengal during the waning 2015 southwest monsoon. Oceanography, 29(2):124-133.

Hosegood, P., Gregg, M. C., and Alford, M. H. (2006). Sub-mesoscale lateral density structure in the oceanic surface mixed layer. Geophysical Research Letters, 33(22).

Itoh, S. and Rudnick, D. L. (2017). Fine-scale variability of isopycnal salinity in the california current system. Journal of Geophysical Research: Oceans, 122(9):7066-7081.

Jaeger, G. S. (2018). Can we improve monsoon forecasts? The ocean may be a key missing link. Oceanus, 53(2):40-44.

Jaeger, G. S. and Mahadevan, A. (2018). Submesoscale-selective compensation of fronts in a salinity-stratified ocean. Science Advances, 4(2):e1701504.

Jinadasa, S., Lozovatsky, I., Planella-Morató, J., Nash, J. D., MacKinnon, J. A., Lucas, A. J., Wijesekera, H. W., and Fernando, H. J. (2016). Ocean turbulence and mixing around Sri Lanka and in adjacent waters of the northern Bay of Bengal. Oceanography, 29(2):170-179. 
Johnson, G. C., Schmidtko, S., and Lyman, J. M. (2012). Relative contributions of temperature and salinity to seasonal mixed layer density changes and horizontal density gradients. Journal of Geophysical Research: Oceans, 117(C4):C04015.

Johnston, T. S., Chaudhuri, D., Mathur, M., Rudnick, D. L., Sengupta, D., Simmons, H. L., Tandon, A., and Venkatesan, R. (2016). Decay mechanisms of near-inertial mixed layer oscillations in the Bay of Bengal. Oceanography, 29(2):180-191.

Kalnay, E., Kanamitsu, M., Kistler, R., Collins, W., Deaven, D., Gandin, L., Iredell, M., Saha, S., White, G., Woollen, J., et al. (1996). The ncep/ncar 40-year reanalysis project. Bulletin of the American Meteorological Society, 77(3):437-471.

Klein, P., Treguier, A.-M., and Hua, B. L. (1998). Three-dimensional stirring of thermohaline fronts. Journal of Marine Research, 56(3):589-612.

Klymak, J. M., Crawford, W., Alford, M. H., MacKinnon, J. A., and Pinkel, R. (2015). Along-isopycnal variability of spice in the north Pacific. Journal of Geophysical Research: Oceans, 120(3):2287-2307.

Kumar, S. P., Ramaiah, N., Gauns, M., Sarma, V., Muraleedharan, P., Raghukumar, S., Kumar, M. D., and Madhupratap, M. (2001). Physical forcing of biological productivity in the northern arabian sea during the northeast monsoon. Deep Sea Research Part II: Topical Studies in Oceanography, 48(6):1115-1126.

Kunze, E., Klymak, J., Lien, R.-C., Ferrari, R., Lee, C., Sundermeyer, M., and Goodman, L. (2015). Submesoscale water-mass spectra in the Sargasso Sea. Journal of Physical Oceanography, 45(5):1325-1338.

Kunze, E. and Sundermeyer, M. A. (2015). The role of intermittency in internal-wave shear dispersion. Journal of Physical Oceanography, 45(12):2979-2990.

Lagerloef, G., Wentz, F., Yueh, S., Kao, H., Johnson, G., and Lyman, J. (2012). Aquarius satellite mission provides new, detailed view of sea surface salinity. Bull. Am. Meteorol. Soc, 93(7):S70-S71.

Lapeyre, G. and Klein, P. (2006). Dynamics of the upper oceanic layers in terms of surface quasigeostrophy theory. Journal of Physical Oceanography, 36(2):165-176.

Lévy, M., Ferrari, R., Franks, P. J., Martin, A. P., and Rivière, P. (2012). Bringing physics to life at the submesoscale. Geophysical Research Letters, 39(14).

Lévy, M., Franks, P. J., and Smith, K. S. (2018). The role of submesoscale currents in structuring marine ecosystems. Nature Communications, 9(1):4758.

Lévy, M., Klein, P., and Treguier, A.-M. (2001). Impact of sub-mesoscale physics on production and subduction of phytoplankton in an oligotrophic regime. Journal of Marine Research, 59(4):535-565.

Lucas, A., Shroyer, E., Wijesekera, H., Fernando, H., D’Asaro, E., Ravichandran, M., Jinadasa, S., MacKinnon, J., Nash, J., Sharma, R., et al. (2014). Mixing to monsoons: Airsea interactions in the Bay of Bengal. Eos, Transactions American Geophysical Union, 95(30):269-270. 
Lucas, A. J., Nash, J. D., Pinkel, R., MacKinnon, J. A., Tandon, A., Mahadevan, A., Omand, M. M., Freilich, M., Sengupta, D., Ravichandran, M., et al. (2016). Adrift upon a salinity-stratified sea: A view of upper-ocean processes in the Bay of Bengal during the southwest monsoon. Oceanography, 29(2):134-145.

MacKinnon, J., Nash, J., Alford, M., Lucas, A., Mickett, J., Shroyer, E., Waterhouse, A., Tandon, A., Sengupta, D., Mahadevan, A., Ravichandran, M., Pinkel, R., Rudnick, D., Whalen, C., Alberty, M., Lekha, J. S., Fine, E., Chaudhuri, D., and Wagner, G. (2016). A tale of two spicy seas. Oceanography, 29(2):50-61.

MacKinnon, J., St Laurent, L., and Garabato, A. C. N. (2013). Diapycnal mixing processes in the ocean interior. In Ocean Circulation and Climate, pages 159-183. Academic Press.

MacVean, M. and Woods, J. (1980). Redistribution of scalars during upper ocean frontogenesis: A numerical model. Quarterly Journal of the Royal Meteorological Society, 106(448):293-311.

Mahadevan, A. (2006). Modeling vertical motion at ocean fronts: Are nonhydrostatic effects relevant at submesoscales? Ocean Modelling, 14(3-4):222-240.

Mahadevan, A., D'Asaro, E., Lee, C., and Perry, M. J. (2012). Eddy-driven stratification initiates north Atlantic spring phytoplankton blooms. Science, 337(6090):54-58.

Mahadevan, A., Oliger, J., and Street, R. (1996). A nonhydrostatic mesoscale ocean model. Part I: Well-posedness and scaling. Journal of Physical Oceanography, 26(9):1868-1880.

Mahadevan, A., Spiro Jaeger, G., Freilich, M., Omand, M., Shroyer, E., and Sengupta, D. (2016). Freshwater in the Bay of Bengal: Its fate and role in air-sea heat exchange. Oceanography, 29(2):72-81.

McCaffrey, K., Fox-Kemper, B., and Forget, G. (2015). Estimates of ocean macroturbulence: Structure function and spectral slope from Argo profiling floats. Journal of Physical Oceanography, 45(7):1773-1793.

McPhaden, M. J. (2002). Mixed layer temperature balance on intraseasonal timescales in the equatorial Pacific ocean. Journal of Climate, 15(18):2632-2647.

McWilliams, J. C. (2016). Submesoscale currents in the ocean. Proc. R. Soc. A, 472(2189):20160117.

McWilliams, J. C. (2017). Submesoscale surface fronts and filaments: secondary circulation, buoyancy flux, and frontogenesis. Journal of Fluid Mechanics, 823:391-432.

Meissner, T., Wentz, F., and Manaster, A. (2018). Remote Sensing Systems SMAP Ocean Surface Salinities [Level 3 Running 8-day], Version 3.0 validated release.

Meunier, T., Ménesguen, C., Schopp, R., and Le Gentil, S. (2015). Tracer stirring around a meddy: The formation of layering. Journal of Physical Oceanography, 45(2):407-423.

Mukherjee, S., Ramachandran, S., Tandon, A., and Mahadevan, A. (2016). Production and destruction of eddy kinetic energy in forced submesoscale eddy-resolving simulations. Ocean Modelling, 105:44-59. 
Munk, W. and Warren, B. (1981). Internal waves and small-scale processes, Evolution of Physical Oceanography, 264-291.

Nagai, T., Tandon, A., Kunze, E., and Mahadevan, A. (2015). Spontaneous generation of near-inertial waves by the Kuroshio front. Journal of Physical Oceanography, 45(9):23812406 .

Pinkel, R., Buijsman, M., and Klymak, J. M. (2012). Breaking topographic lee waves in a tidal channel in Luzon strait. Oceanography, 25(2):160-165.

Pinkel, R., Rainville, L., Slater, E., Goldin, A., Green, L., Bui, M., and Aja, T. (2003). The hydrographic doppler sonar system on the roger revelle. In Current Measurement Technology, 2003. Proceedings of the IEEE/OES Seventh Working Conference, pages 237239. IEEE.

Poje, A. C., Özgökmen, T. M., Lipphardt, B. L., Haus, B. K., Ryan, E. H., Haza, A. C., Jacobs, G. A., Reniers, A., Olascoaga, M. J., Novelli, G., et al. (2014). Submesoscale dispersion in the vicinity of the Deepwater Horizon spill. Proceedings of the National Academy of Sciences, 111(35):12693-12698.

Polzin, K. and Ferrari, R. (2004). Isopycnal dispersion in NATRE. Journal of Physical Oceanography, 34(1):247-257.

Rainville, L. and Pinkel, R. (2004). Observations of energetic high-wavenumber internal waves in the Kuroshio. Journal of Physical Oceanography, 34(7):1495-1505.

Ramachandran, S., Tandon, A., Mackinnon, J., Lucas, A. J., Pinkel, R., Waterhouse, A. F., Nash, J., Shroyer, E., Mahadevan, A., Weller, R. A., et al. (2018). Submesoscale processes at shallow salinity fronts in the Bay of Bengal: Observations during the winter monsoon. Journal of Physical Oceanography, 48(3):479-509.

Rao, R. R. and Sivakumar, R. (2003). Seasonal variability of sea surface salinity and salt budget of the mixed layer of the north Indian Ocean. Journal of Geophysical Research: Oceans, 108(C1):3009.

Richards, K. and Edwards, N. (2003). Lateral mixing in the equatorial Pacific: The importance of inertial instability. Geophysical Research Letters, 30(17).

Ruddick, B. (1983). A practical indicator of the stability of the water column to doublediffusive activity. Deep Sea Research Part A. Oceanographic Research Papers, 30(10):11051107.

Ruddick, B. and Turner, J. (1979). The vertical length scale of double-diffusive intrusions. Deep Sea Research Part A. Oceanographic Research Papers, 26(8):903-913.

Rudnick, D. L. and Cole, S. T. (2011). On sampling the ocean using underwater gliders. Journal of Geophysical Research: Oceans, 116(C8).

Rudnick, D. L. and Ferrari, R. (1999). Compensation of horizontal temperature and salinity gradients in the ocean mixed layer. Science, 283(5401):526-529.

Rudnick, D. L. and Martin, J. P. (2002a). On the horizontal density ratio in the upper ocean. Dynamics of Atmospheres and Oceans, 36(1-3):3-21. 
Rudnick, D. L. and Martin, J. P. (2002b). On the horizontal density ratio in the upper ocean. Dynamics of Atmospheres and Oceans, 36(1-3):3-21.

Sarkar, S., Pham, H. T., Ramachandran, S., Nash, J. D., Tandon, A., Buckley, J., Lotliker, A. A., and Omand, M. M. (2016). The interplay between submesoscale instabilities and turbulence in the surface layer of the Bay of Bengal. Oceanography, 29(2):146-157.

Schmidtko, S., Johnson, G. C., and Lyman, J. M. (2013). MIMOC: A global monthly isopycnal upper-ocean climatology with mixed layers. Journal of Geophysical Research: Oceans, 118(4):1658-1672.

Schmitt, R. W. (1994). Double diffusion in oceanography. Annual Review of Fluid Mechanics, 26(1):255-285.

Schmitt, R. W., Perkins, H., Boyd, J., and Stalcup, M. (1987). C-SALT: an investigation of the thermohaline staircase in the western tropical north atlantic. Deep Sea Research Part A. Oceanographic Research Papers, 34(10):1655-1665.

Schott, F. A. and McCreary Jr, J. P. (2001). The monsoon circulation of the Indian Ocean. Progress in Oceanography, 51(1):1-123.

Sengupta, D., Bharath Raj, G., and Shenoi, S. (2006). Surface freshwater from Bay of Bengal runoff and Indonesian throughflow in the tropical Indian Ocean. Geophysical Research Letters, 33(22).

Sengupta, D., Bharath Raj, G. N., Ravichandran, M., Sree Lekha, J., and Papa, F. (2016). Near-surface salinity and stratification in the north Bay of Bengal from moored observations. Geophysical Research Letters, 43(9):2016GL068339.

Seo, H., Xie, S.-P., Murtugudde, R., Jochum, M., and Miller, A. J. (2009). Seasonal effects of Indian Ocean freshwater forcing in a regional coupled model. Journal of Climate, 22(24):6577-6596.

Shcherbina, A. Y., Gregg, M. C., Alford, M. H., and Harcourt, R. R. (2009). Characterizing thermohaline intrusions in the north Pacific subtropical frontal zone. Journal of Physical Oceanography, 39(11):2735-2756.

Shcherbina, A. Y., Sundermeyer, M. A., Kunze, E., D’Asaro, E., Badin, G., Birch, D., Brunner-Suzuki, A.-M. E., Callies, J., Kuebel Cervantes, B. T., Claret, M., et al. (2015). The LatMix summer campaign: submesoscale stirring in the upper ocean. Bulletin of the American Meteorological Society, 96(8):1257-1279.

Shenoi, S., Shankar, D., and Shetye, S. (2002). Differences in heat budgets of the nearsurface arabian sea and Bay of Bengal: Implications for the summer monsoon. Journal of Geophysical Research: Oceans, 107(C6):5-1.

Shetye, S. R., Gouveia, A. D., Shankar, D., Shenoi, S. S. C., Vinayachandran, P. N., Sundar, D., Michael, G. S., and Nampoothiri, G. (1996). Hydrography and circulation in the western Bay of Bengal during the northeast monsoon. Journal of Geophysical Research: Oceans, 101(C6):14011-14025. 
Shroyer, E., Rudnick, D., Farrar, J. T., Lim, B., Venayagamoorthy, S. K., St. Laurent, L., Garanaik, A., and Moum, J. (2016a). Modification of upper-ocean temperature structure by subsurface mixing in the presence of strong salinity stratification. Oceanography, $29(2): 62-71$.

Shroyer, E. L., Rudnick, D. L., Farrar, J. T., Lim, B., Venayagamoorthy, S. K., St. Laurent, L. C., Garanaik, A., and Moum, J. N. (2016b). Modification of upper-ocean temperature structure by subsurface mixing in the presence of strong salinity stratification. Oceanography, 29(2):62-71.

Slingo, J., Inness, P., Neale, R., Woolnough, S., Yang, G., et al. (2003). Scale interactions on diurnal toseasonal timescales and their relevanceto model systematic errors. Annals of Geophysics.

Smith, K. S. and Ferrari, R. (2009). The production and dissipation of compensated thermohaline variance by mesoscale stirring. Journal of Physical Oceanography, 39(10):24772501.

Sree Lekha, J., Buckley, J., Tandon, A., and Sengupta, D. (2018). Subseasonal dispersal of freshwater in the northern Bay of Bengal in the 2013 summer monsoon season. Journal of Geophysical Research: Oceans, 123(9):6330-6348.

Stommel, H. (1962). On the cause of the temperature-salinity curve in the ocean. Proceedings of the National Academy of Sciences, 48(5):764-766.

Sun, H. and Kunze, E. (1999). Internal wave-wave interactions. part i: The role of internal wave vertical divergence. Journal of Physical Oceanography, 29(11):2886-2904.

Sundermeyer, M. A. and Ledwell, J. R. (2001). Lateral dispersion over the continental shelf: Analysis of dye release experiments. Journal of Geophysical Research: Oceans, 106(C5):9603-9621.

Sundermeyer, M. A., Ledwell, J. R., Oakey, N. S., and Greenan, B. J. (2005). Stirring by small-scale vortices caused by patchy mixing. Journal of Physical Oceanography, 35(7):1245-1262.

Thangaprakash, V., Girishkumar, M., Suprit, K., Kumar, N. S., Chaudhuri, D., Dinesh, K., Kumar, A., Shivaprasad, S., Ravichandran, M., Farrar, J. T., et al. (2016). What controls seasonal evolution of sea surface temperature in the Bay of Bengal? mixed layer heat budget analysis using moored buoy observations along $90^{\circ}$ E. Oceanography.

Thomas, L. N., Tandon, A., and Mahadevan, A. (2008a). Submesoscale Processes and Dynamics. In Hecht, t. W. and Hasumi, H., editors, Ocean Modeling in an Eddying Regime, pages 17-38. American Geophysical Union.

Thomas, L. N., Tandon, A., and Mahadevan, A. (2008b). Submesoscale processes and dynamics. Ocean Modeling in an Eddying Regime, 177:17-38.

Thompson, A. F., Lazar, A., Buckingham, C., Naveira Garabato, A. C., Damerell, G. M., and Heywood, K. J. (2016). Open-ocean submesoscale motions: A full seasonal cycle of mixed layer instabilities from gliders. Journal of Physical Oceanography, 46(4):1285-1307. 
Thomson, R. E. and Emery, W. J. (2014). Data Analysis Methods in Physical Oceanography. Elsevier.

Timmermans, M.-L. and Winsor, P. (2013). Scales of horizontal density structure in the Chukchi Sea surface layer. Continental Shelf Research, 52:39-45.

Tompkins, A. M. (2001). On the relationship between tropical convection and sea surface temperature. Journal of Climate, 14(5):633-637.

Toole, J. M. and McDougall, T. J. (2001). Chapter 5.2 Mixing and stirring in the ocean interior. In International Geophysics, pages 337-355. Elsevier.

Vallis, G. K. (2017). Atmospheric and Oceanic Fluid Dynamics. Cambridge University Press.

Webster, P. J., Magana, V. O., Palmer, T., Shukla, J., Tomas, R., Yanai, M., and Yasunari, T. (1998). Monsoons: Processes, predictability, and the prospects for prediction. Journal of Geophysical Research: Oceans, 103(C7):14451-14510.

Whitt, D. B. and Thomas, L. N. (2013). Near-inertial waves in strongly baroclinic currents. Journal of Physical Oceanography, 43(4):706-725.

Wijesekera, H. W., Shroyer, E., Tandon, A., Ravichandran, M., Sengupta, D., Jinadasa, S., Fernando, H. J., Agrawal, N., Arulananthan, K., Bhat, G., et al. (2016). ASIRI: An ocean-atmosphere initiative for Bay of Bengal. Bulletin of the American Meteorological Society, 97(10):1859-1884.

Young, W., Rhines, P., and Garrett, C. (1982). Shear-flow dispersion, internal waves and horizontal mixing in the ocean. Journal of Physical Oceanography, 12(6):515-527.

Yu, L., Jin, X., and Weller, R. A. (2008). Multidecade global flux datasets from the Objectively Analyzed Air-sea Fluxes (OAFlux) project: Latent and sensible heat fluxes, ocean evaporation, and related surface meteorological variables. OAFlux Project Tech. Rep. OA-2008-01., 74. 